\title{
In vitro models for the prediction of in vivo performance of oral dosage forms
}

Edmund S. Kostewicz ${ }^{\mathrm{a}}$, Bertil Abrahamsson ${ }^{\mathrm{b}}$, Marcus Brewster ${ }^{\mathrm{c}}$, Joachim Brouwers ${ }^{\mathrm{d}}$, James Butler $^{\mathrm{e}}$, Sara Carlert ${ }^{\mathrm{b}}$, Paul A. Dickinson ${ }^{\mathrm{f}}$, Jennifer Dressman ${ }^{\mathrm{a}}$, René Holm ${ }^{\mathrm{g}}$, Sandra Klein ${ }^{\mathrm{h}}$, James Mann ${ }^{\mathrm{i}}$, Mark McAllister ${ }^{\mathrm{j}}$, Mans Minekus ${ }^{\mathrm{k}}$, Uwe Muenster ${ }^{\mathrm{l}}$, Anette Müllertz ${ }^{\mathrm{m}}$, Miriam Verwei $^{\mathrm{k}}$, Maria Vertzoni ${ }^{\mathrm{n}}$, Werner Weitschies ${ }^{\mathrm{h}}$, Patrick Augustijns ${ }^{\mathrm{d}}$

${ }^{a}$ Institute of Pharmaceutical Technology, Goethe University, Frankfurt/Main, Germany

${ }^{\mathrm{b}}$ Department of Global Medicines Development, AstraZeneca R\&D, S-431 83 Mölndal, Sweden

${ }^{c}$ Drug Product Development, Pharmaceutical Development and Manufacturing Sciences Janssen Research and Development, Johnson \& Johnson, Beerse, Belgium

${ }^{\mathrm{d}}$ Drug Delivery and Disposition, KU Leuven, Leuven, Belgium

${ }^{\mathrm{e}}$ Product Development, GlaxoSmithKline R\&D, Harlow, UK

${ }^{\mathrm{f}}$ Clinical Pharmacology and Pharmacometrics, AstraZeneca R\&D, Alderley Park, Macclesfield, United Kingdom SK10 4TG

${ }^{\mathrm{g}}$ Biologics and Pharmaceutical Science, H.Lundbeck A/S, Ottiliavej 9, DK 2500 Valby Denmark

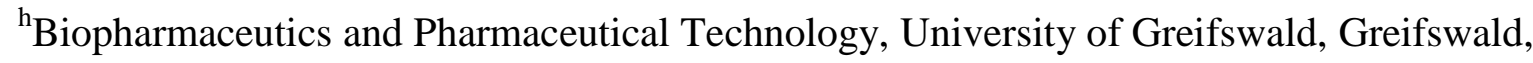
Germany

${ }^{\mathrm{i}}$ Development Laboratories, MSD, Hoddesdon, UK

${ }^{\mathrm{jj}}$ Drug Product Design, Pfizer PGRD, Sandwich, Kent, CT13 9NJ, U.K.

${ }^{\mathrm{k}} \mathrm{TNO}$, Zeist, The Netherlands

${ }^{\mathrm{l}}$ Material Science and Early Characterization, Research Center Aprath, Bayer Pharma AG, Germany

${ }^{\mathrm{m}}$ Pharmaceutical Design and Drug Delivery, University of Copenhagen, Denmark

${ }^{\mathrm{n}}$ Faculty of Pharmacy, National and Kapodistrian University of Athens, Panepistimiopolis 15771 Athens, Greece 
Corresponding author

Patrick Augustijns

Drug Delivery and Disposition

O\&N 2, Box 921, KU Leuven, Belgium

\section{Abbreviations}

Biopharmaceutics Classification System (BCS)

Extended Release (ER)

Immediate Release (IR)

Innovative Medicines Initiative (IMI)

In vitro in Vivo Correlation (IVIVC)

Modified Release (MR)

Oral Biopharmaceutical Tools (OrBiTo)

Pharmacokinetics (PK)

Physiologically-based pharmacokinetics (PBPK)

Quality by Design (QbD)

Quality Control (QC)

U.S Food and Drug Administration (FDA)

United States Pharmacopeia (USP)

\section{Keywords}

in vitro evaluation; oral drug formulations; in vivo biopharmaceutical performance; biorelevant; Innovative Medicines Initiative (IMI); supersaturation and precipitation; solubility-permeability interplay; in vitro biopharmaceutical tools; Oral Biopharmaceutical Tools (OrBiTo) 


\section{Abstract}

Accurate prediction of the in vivo biopharmaceutical performance of oral drug formulations is critical to efficient drug development. Traditionally, in vitro evaluation of oral drug formulations has focused on disintegration and dissolution testing for quality control (QC) purposes. The connection with in vivo biopharmaceutical performance has often been ignored. More recently, the switch to assessing drug products in a more biorelevant and mechanistic manner has advanced the understanding of drug formulation behavior. Notwithstanding this evolution, predicting the in vivo biopharmaceutical performance of formulations that rely on complex intraluminal processes (e.g. solubilization, supersaturation, precipitation...) remains extremely challenging. Concomitantly, the increasing demand for complex formulations to overcome low drug solubility or to control drug release rates urges the development of new in vitro tools. Development and optimizing innovative, predictive Oral Biopharmaceutical Tools is the main target of the OrBiTo project within the Innovative Medicines Initiative (IMI) framework. A combination of physico-chemical measurements, in vitro tests, in vivo methods, and physiology-based pharmacokinetic modeling is expected to create a unique knowledge platform, enabling the bottlenecks in drug development to be removed and the whole process of drug development to become more efficient.

As part of the basis for the OrBiTo project, this review summarizes the current status of predictive in vitro assessment tools for formulation behaviour. Both pharmacopoeia-listed apparatus and more advanced tools are discussed. Special attention is paid to major issues limiting the predictive power of traditional tools, including the simulation of dynamic changes in gastrointestinal conditions, the adequate reproduction of gastrointestinal motility, the simulation of supersaturation and precipitation, and the implementation of the solubilitypermeability interplay. It is anticipated that the innovative in vitro biopharmaceutical tools arising from the OrBiTo project will lead to improved predictions for in vivo behavior of drug formulations in the GI tract. 


\section{Table of Contents}

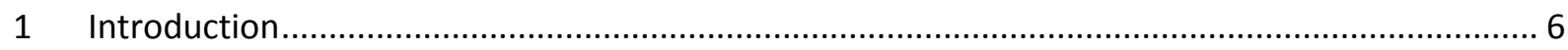

1.1 The challenging nature of oral drug products today ....................................................... 6

1.2 The historic connection between drug release testing and in vivo performance................... 8

2 Pharmacopoeial setups - how useful are they for development? ............................................. 10

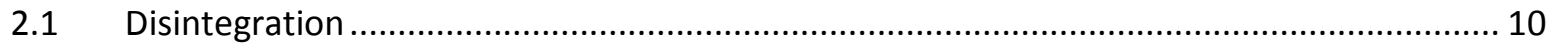

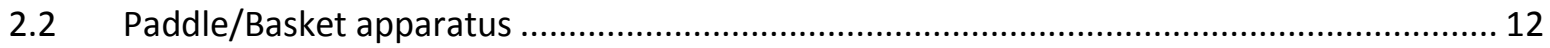

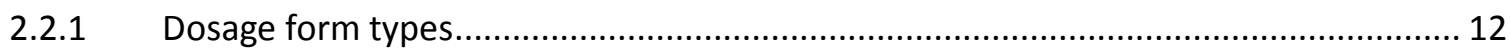

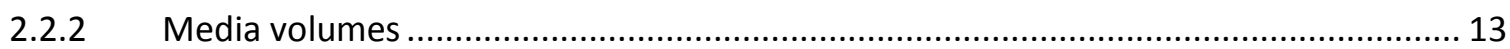

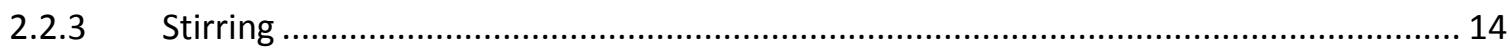

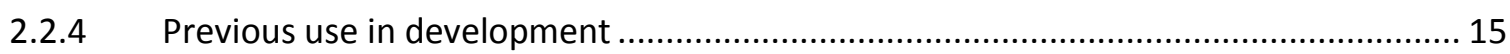

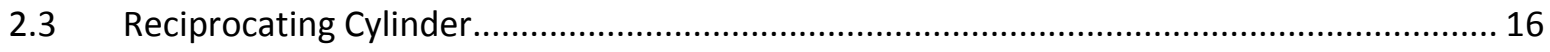

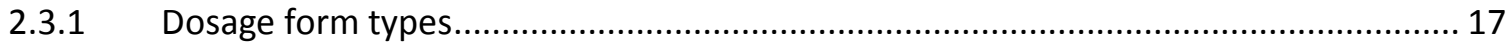

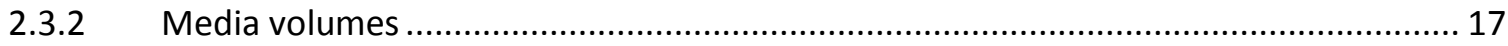

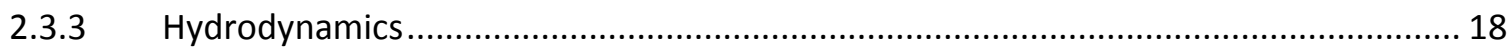

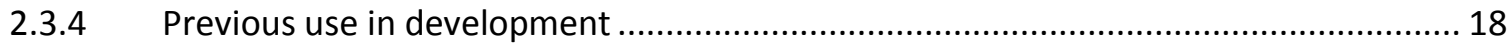

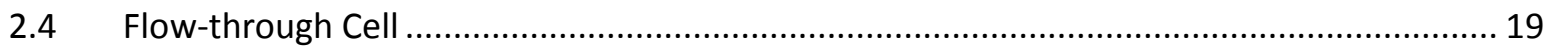

3 Overcoming the limitations of pharmacopoeial setups - recent advances and current challenges 22

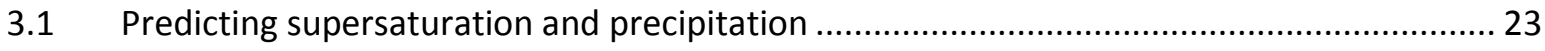

3.2 Accounting for absorption in formulation evaluation....................................................... 27

3.2.1 Assessment of permeation from relevant samples.................................................... 28

3.2.2 Integrating absorption in in vitro dissolution and precipitation setups........................ 28

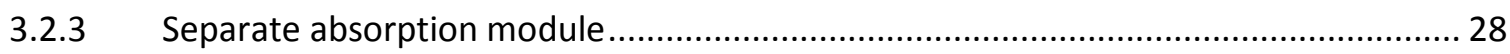

3.2.4 Direct integration of absorption in the dissolution module......................................... 29

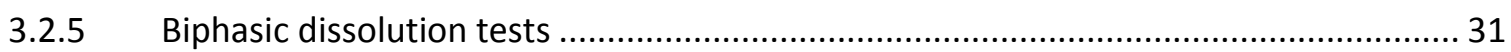

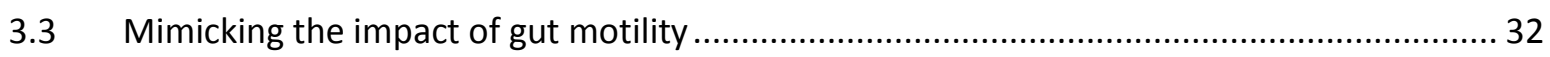

3.3.1 Importance of hydrodynamics for drug release and dissolution .................................. 32

3.3.2 Attempts to model the impact of GI motility in dissolution testing .............................. 33

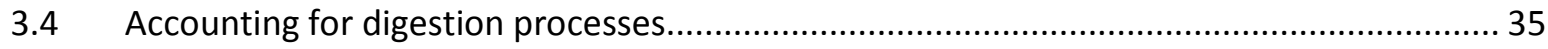

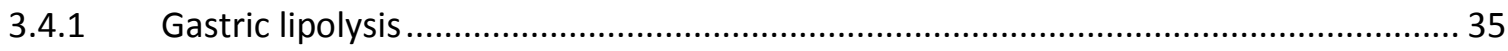

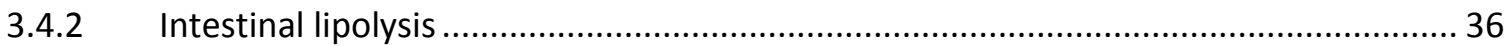


3.5 Dynamic multi-compartmental "GI tract in the lab" systems

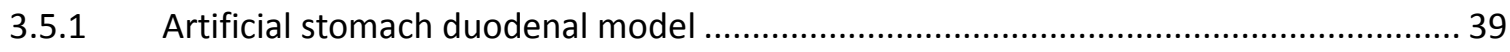

3.5.2 Dynamic Gastric Model ....................................................................... 40

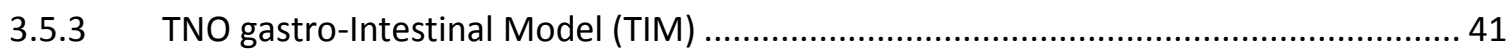

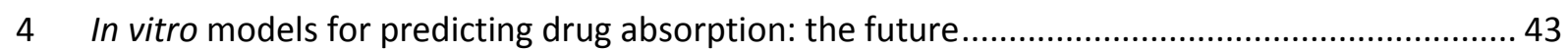

$5 \quad$ Integration of in vitro models with PBPK modeling ........................................................ 47

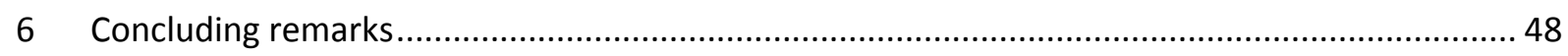

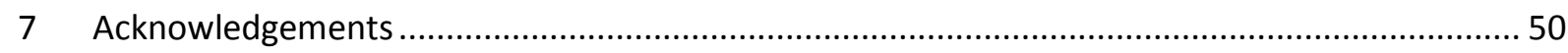

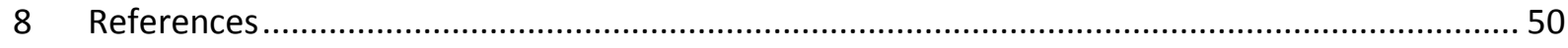

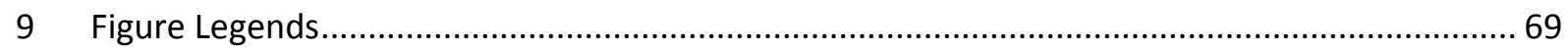

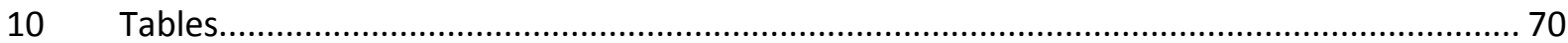




\section{Introduction}

\subsection{The challenging nature of oral drug products today}

The research-based pharmaceutical industry is currently facing unprecedented pressures, in large part due to declining return on investment from $R \& D$ over the last decade or so.

The latest figures for new drug approvals in the USA reflect some of current challenges and how the industry is responding to these. In 2012, there were approximately 40 new drug approvals (Center for Drug Evaluation and Research, 2013), which encouragingly is the highest number in 16 years. However, the total anticipated peak sales for these new drugs is lower compared to that of new approvals in previous years ("Measuring the return from Pharmaceutical innovation 2012 - Deloitte UK Centre for Health Solutions | Deloitte UK," 2013). This reflects a trend towards a higher proportion of medicines being developed (or at least succeeding in reaching the market) for niche patient populations, where unmet medical need is high and the chances of getting a drug to the market is improved. As a result, for these products, the return on investment per molecule launched is somewhat lower compared to the traditional so-called "blockbuster" products where patient numbers per molecule are higher. Furthermore, a significant challenge for the introduction of innovative products in precedented therapeutic areas is the ready availability of once class-leading products in generic form. This is perhaps best exemplified in the latest wave of generic introductions by the availability of now generic atorvastatin providing prescribers with an additional alternative to statins still under patent protections. Overall, these challenges make it even more difficult to justify the development of innovative products. To ensure that the industry remains commercially viable, the drug product development process needs to become more efficient.

In concordance with the recent shifts in the types of diseases being addressed by new drugs and the increase in development failures in certain indications [e.g. CNS, Oncology (Kola, 2008) ], the pipelines of pharmaceutical companies today contain a higher proportion of drugs with significant challenges in terms of drug delivery and biopharmaceutics. Looking at development pipelines of pharmaceutical industry over the past five decades (Gribbon and Sewing, 2005; Lobell e.a., 2006), biopharmaceutical issues are mostly related to increasing lipophilicity and decreasing aqueous solubility. As a consequence, more development candidates exhibit poor oral bioavailability from oral drug formulations containing the crystalline form of the API. On the other hand, the lipophilicity of marketed compounds 
remained fairly unchanged over that time-span, indicating that the changes in the physicochemical properties of compounds in pipelines has probably been detrimental to the chances of successful development (Arrowsmith, 2011a). Reasons for the observed increase in the number of lipophilic compounds in development include:

i) The addition of lipophilic residues to achieve an increased ligand-receptor affinity (Vieth et al., 2004);

ii) A general broadening of chemical space, supported by the launch of combinatorial chemistry in the early 1990s (Moos et al., 1993; Patel and Gordon, 1996);

iii) The introduction of high-through-put screening (HTS) in the early 1990s, which led to a bias towards lipophilic compounds being identified as potential leads.

Given the increasingly unfavorable biopharmaceutical properties of development candidates, formulation scientists have had to develop a variety of strategies over the past decades in order to overcome them. For instance, drugs are being formulated for oral immediate release (IR) in products that deliberately create supersaturation and/or increase of the drug's solubility at some point in the GI tract. Such approaches are now widespread in the modern oral pharmaceutical portfolio and include solid dispersions, self microemulsifying systems, salts and cocrystals (Williams et al., 2013).

One way of building greater efficiency into the drug development process is to switch from the traditional empirical approach of product development and make greater use of predictive tools based upon a sound scientific understanding of in vivo behavior. Since lack of efficacy and safety concerns are the largest contributors to attrition (J Arrowsmith, 2011a, b; Kola and Landis, 2004), this scientific and predictive approach is especially important when understanding therapeutic drug targets, mechanisms causing toxicological effects and drugdrug interactions. Biopharmaceutics and pharmacokinetics (PK) are also a direct cause of attrition, although there is some evidence of improvement in recent decades (Astashkina et al., 2012). In addition, candidates with poor biopharmaceutic and pharmacokinetic properties tend to take more time and effort to progress through development, and may make safety and efficacy failures more likely (Hann and Keserü, 2012). Whilst the industry has taken on board and successfully implemented screens to remove compounds with Cytochrome P450mediated metabolic liabilities, there are reports which suggest a shift to compounds for which 
the in vitro screens are not as predictive for phase II pathways such as glucuronidation (Miners et al., 2006).

To help drive this much needed switch to more efficient development, and to encourage synergies between pharmaceutical companies in Europe, IMI [Innovative Medicines Initiative ("Home | IMI - Innovative Medicines Initiative," 2013)] has implemented 37 public-private partnerships with more to come. These are research collaborations between Pharmaceutical Companies, Universities, Patient Organizations, Medical Agencies etc., focusing on the most urgent bottlenecks in Pharmaceutical R\&D. Of these, the Oral Biopharmaceutics Tools (OrBiTo) project aims to create new and optimized laboratory tests and computer models that will better predict the biopharmaceutical performance of drugs and formulations in patients as well as providing suggestions for the most rational use of novel and existing methods. The aims and objectives of OrBiTo also relate well to the FDA's Quality by Design (QbD) initiative, which is designed to encourage a better understanding of new products during development, including developing and understanding in vivo behavior (ICH Expert Working Group, 2009; Yu, 2008).

In light of the OrBiTo IMI initiative, this article is a review and gap analysis of current knowledge in the biopharmaceutical field, with special emphasis on in vitro tools to predict in vivo performance of pharmaceutical formulations.

While many innovative options for delivering low solubility drugs have been developed, novel and reliable in vitro tests to predict the in vivo dissolution, precipitation and/or absorption of the drug from these formulations are in need of further development. Traditional dissolution using the paddle or basket apparatus is normally utilized to assess product quality and to predict changes in drug release that may impact the in vivo performance of oral products, but has not changed radically since the 1970s. Limitations of the traditional approach to dissolution are of particular relevance to low solubility drugs with their challenging biopharmaceutical properties

\subsection{The historic connection between drug release testing and in vivo performance}

More than a hundred years ago, the importance of dissolution testing to in vivo performance had already been recognized. Quoting C. Caspari in 1895

“ ....it would seem that prompt action of certain remedies must be considerably impaired by firm compression. The composition of all compressed tablets 
should be such that they will readily undergo disintegration and dissolution in the stomach." (C. Caspari, A treatise on Pharmacy 1895, Lea Bros.

Philadelphia p344.)

As described by Krämer (Dressman and Krämer, 2005), the evolution of suitable methods to establish links between in vitro and in vivo product performance had to be put on hold until reliable analytical methods were developed in the 1950s. In that era, vitamin products and enteric coated tablets came under closer scrutiny, and failure to disintegrate was linked to poor clinical results by greats such as John Wagner, the leading pharmacokineticist of the day. In the 1970s, as generic drug products became common, interest in linking in vitro with in vivo performance intensified and in 1973 a correlation between dissolution and absorption rates was demonstrated for digoxin tablets (Shaw et al., 1973). This paved the way for the field of in vitro in vivo correlation (IVIVC). In the 1970s and 1980s, many attempts were made to establish IVIVCs for various types of drug products, as summarized by Welling (Welling et al., 1991). At that time, Welling reported that "to the writer's knowledge, there have been no studies that have accurately correlated in vitro and in vivo data to the point that the use of upper and lower limits for in vitro dissolution parameters can be confidently used to predict in vivo behavior and therefore to replace in vivo testing". However, both the pharmaceutical industry and the regulatory authorities persisted with the IVIVC concept, holding workshops and drafting chapters on IVIVC during the 1980s (Pharmacopeial Forum, 1988; "PMA comments on USP Stimuli to the revision process. In vitro-in vivo correlations for extended release oral dosage forms," 1988; PMA Joint Committee on Bioavailability, 1985; Skelley et al., 1990). IVIVC had to wait, however, until 1995 to be included in the United States Pharmacopeia. In USP Chapter 1088, it was stated that "the goal of the pharmaceutical scientist is to find a relationship between an in vitro characteristic of a dosage form and its in vivo performance" (US Pharmacopoeia, 1995). By that time, it was recognized that an IVIVC can be more readily defined for MR than for IR dosage forms. In 1997, guidance issued by the FDA described the application of IVIVC in the development and evaluation of extended release (ER) dosage forms (Malinowski et al., 1997). Two years later, IVIVC was mentioned in the corresponding European Note for Guidance ("GMP Guideline Quality of Modified Release Products Section I (Quality) CPMP/QWP/ 604/96,” 2013). Over the following decade, the IVIVCs generated have predominantly been for MR dosage forms, which is not unexpected as by definition dissolution should be rate determining for the PK profile. 
With more poorly soluble drugs being developed as oral dosage forms, it was recognized in the 2000s that, since the dissolution is often an important restriction to their absorption, IVIVC might be possible for drug products containing poorly soluble drugs, even if the product was intended for "immediate" release.

\section{Pharmacopoeial setups - how useful are they for development?}

\subsection{Disintegration}

The disintegration test is performed to determine whether tablets or capsules disintegrate within the prescribed time when placed in a liquid medium at $37^{\circ} \mathrm{C}$ using the disintegration apparatus and experimental conditions proposed by the Pharmacopeia (The European Pharmacopoeia, 2011; United States Pharmacopoeia Convention, 2012). According to the Pharmacopeias, disintegration is defined as that state in which any residue of the unit, except from fragments of insoluble coating or capsule shell, remaining on the screen of the test apparatus or adhering to the lower surface of the disk, if used, is a soft mass having no palpable core (The European Pharmacopoeia, 2011). Compliance with the limits on disintegration in the individual monograph is required except where the label states that the tablets or capsules are intended for use as troches, are to be chewed, or are designed as ER or delayed release dosage forms (The European Pharmacopoeia, 2011). The apparatus consists of a basket-rack assembly, a $1000 \mathrm{~mL}$ low-form beaker, a thermostatic water bath and a device for raising and lowering the basket in the immersion fluid at a constant frequency. Disintegration tests are performed with water or USP simulated gastric fluid as the immersion fluids, except when evaluating enteric coated tablets in which case USP simulated gastric fluid is used for one hour followed by USP simulated intestinal fluid for the time period specified in each monograph.

These QC disintegration test conditions often deviate from the physiology in the gastrointestinal tract and neglect the dosing conditions used. For example, when conducting a disintegration test for orally disintegrating (or orodispersible) tablets (ODTs), the volume used is substantially greater than the physiologically relevant volumes.

According to the FDA Guidance for Industry (CDER, 2008), ODTs are considered solid oral preparations that disintegrate rapidly in the oral cavity with an in vitro disintegration time of approximately 30 seconds, or less; determination of disintegration time in vitro may be problematic as it has a subjective endpoint, especially for products that form pulpy masses or 
create cloudy solutions (Brown et al., 2011). Additionally, disintegration tests may not be relevant in cases where the tablets erode quickly into slowly eroding granules; in this case, drug release from the smaller particles may be more appropriate than the results obtained from the disintegration test.

Weitschies et al. (2001) have shown that, when the intragastric disintegration behavior of gelatin capsules is rapid, results correspond well to the disintegration times observed in simple aqueous media such as water or hydrochloric solution. By contrast, scintigraphic studies showed that intragastric disintegration times of hard gelatin capsules are delayed in both the fasted and fed states according to the degree of cross-linking (Digenis et al., 2000). These findings were predicted satisfactorily by the in vitro disintegration times estimated in biorelevant media consisting of fasted state simulating fasted fluid (FaSSGF) for the fasted state and in milk gradually digested with pepsin for the fed state (Kalantzi et al., 2008), whereas results were poorly predicted in compendial media (Digenis et al., 2000). Disintegration times in vitro corresponded to the lag times prior to the onset of dissolution in vivo and, in the fed state, both the delayed disintegration and the rank order of disintegration times of three different sets of capsules could be predicted.

In the fed state, $\gamma$ scintigraphy studies have shown that, in humans, the fed state disintegration time of both tablets (Kelly et al., 2003) and capsules with nominal "immediate release" (Cole et al., 2004; Digenis et al., 2000) is substantially prolonged compared to the fasted state disintegration times. A significant food effect on the onset and complete disintegration times of solid dosage forms has also been confirmed using a magnetic imaging technique (Goodman et al., 2010).

Abrahamsson et al. (2004) showed that food could significantly delay tablet disintegration and drug dissolution in the stomach by formation of a film around tablets. This effect could be monitored by a simple in vitro disintegration test using a test medium based on a nutritional drink (Scandishake Mix ${ }^{\circledR}$ ). A similar delay in tablet disintegration was found in vivo after administration of a nutritional drink to dogs and removal of the tablet from the stomach through a gastric fistula. In vitro studies showed that the extent of food interaction on tablet disintegration was dependent on the composition of the meal: the presence of protein (casein) seemed to be critical with respect to this effect. 
Using the dynamic TNO gastroIntestinal Model (TIM-1) and magnetic resonance imaging, Brouwers et al. recently achieved an accurate prediction of impaired postprandial disintegration of fosamprenavir tablets coated with a HPMC film (Brouwers et al., 2011).

\subsection{Paddle/Basket apparatus}

The paddle (Type 2) and basket (Type 1) apparatus (Figure 1) were the first dissolution testers introduced into the Pharmacopeia and are extensively recommended in USP individual monographs for dissolution testing of various kinds of dosage forms. The tests prescribed in these monographs primarily address the QC of the respective drug products. In those cases where QC methods also fulfill QbD goals, these methods may also be applicable to the development of drug products. Examples of drug product types where the QC test might also be useful for product development include IR oral drug products containing drugs belonging to Class I or III of the Biopharmaceutics Classification Scheme (BCS) and drug products with MR where the release rate is very robust to variations in gastrointestinal physiology e.g. many osmotic pump formulations or well-designed coated pellets (Grundy and Foster, 1996; Sandberg et al., 1991). For other types of dosage forms, direct translation of methods from the pharmacopeial recommendation to the development laboratory may be less productive.

The introduction of the BCS in the 1990s, which considers both solubility and permeability as key determinants influencing in vivo performance, had a significant impact on the development of IR dosage formulations. It provided the platform to use in vitro data generated with the paddle/basket apparatus rather than in vivo human studies for establishing bioequivalence of BCS class I compounds (Amidon et al., 1995; Food and Drug Administration, 2009)

\subsubsection{Dosage form types}

In principle, the paddle and basket can be used for all oral dosage forms. With respect to IR products, tablets can generally be tested with either apparatus without additional hardware, whereas capsules often require a sinker to hold the capsule in the medium when tested with the paddle and for this reason the basket apparatus may be preferred. For studying the release characteristics from enteric coated (EC) products, it is often easier to work with the basket when changing from gastric to intestinal media if a "full change" method, i.e. complete removal and replacement of the medium, is to be used. Even if the EC product consists of small pellets, this does not usually create a problem for studies using the basket method. The 
paddle and the basket methods can be suitable for testing MR dosage forms if the formulation is robust to changes in the physiology as its proceeds through the GI tract. Since it is difficult to change the medium more than once with these apparatus, formulation performance that changes with site in the GI tract is harder to track using the paddle or basket method. Thus, the simple methods using either the paddle or basket method that are often used for QC may have less relevance for in vivo because of the lack of flexibility in changing the media composition.

\subsubsection{Media volumes}

Paddle and basket methods usually call for media volumes in the range of 500 to $1,000 \mathrm{~mL}$. Such volumes are useful to generate sink conditions for dissolution of the drug, which are needed to meet one of the key QC goals - i.e. to show that the drug can be completely released from the dosage form. However, these volumes may bear little relevance to the in vivo situation, depending on whether or not the product is to be given with meals. Volumes approaching 1 liter or even greater may be achieved during meal digestion in the stomach, at least in the early-middle phases of digestion. However, should the dosage form be ingested in the fasted state with a glass of water, gastric volumes are unlikely to exceed $250 \mathrm{~mL}$ and thus the volume used in the dissolution test will be too high to accurately reflect conditions in the stomach (Schiller et al., 2005). For BCS I and III drugs, for which sink conditions are likely to prevail even at low volumes, this mismatch in volumes between the apparatus and the fasted stomach is unlikely to be an issue, whereas for poorly soluble drugs, the high volumes used can lead to an overestimation of the dissolution in the stomach in vivo.

With respect to intestinal conditions, the volume is not the only influence on whether sink conditions are generated or not. As drugs are absorbed from this region, sink conditions may be generated via removal of the drug from solution by uptake across the gut wall. Thus, the question of whether it is appropriate to generate sink conditions in the in vitro test will depend on the permeability of the drug as well as its solubility. Thus, for poorly soluble drugs, the volumes used with the paddle and basket apparatus are better suited to dissolution of drugs with high (BCS class II) than low permeability (BCS IV) in the small intestine.

If appropriate to the drug/dosing situation, smaller volumes of dissolution media can be achieved using mini-paddles in combination with specially designed, smaller vessels; in this case, the minimum volume that can be used is approximately $200 \mathrm{~mL}$. This is particularly useful for simulating conditions in the stomach or in the small intestine for dissolution of BCS 
class IV drugs in the fasted state. Klein et al. demonstrated that the mini-paddle set-up is properly hydrodynamically scaled to the paddle set-up (Klein and Shah, 2008). However, the mini-paddle apparatus has not yet been formally included in any of the pharmacopeia and, as a result, the design and dimensions has not yet been harmonized across all manufacturers.

\subsubsection{Stirring}

The hydrodynamic patterns in the paddle apparatus have been studied in detail (Bai et al., 2007; Diebold and Dressman, 2001), and several computer simulation models have been generated to describe the hydrodynamics (McCarthy et al., 2003). The hydrodynamics of standard USP methods is problematic, not only because the in vivo conditions have not been considered in the design of these methods but also because the hydrodynamic conditions vary substantially within the dissolution vessel (Bai et al., 2007; Baxter et al., 2005a; D'Arcy et al., 2005). Thus, depending on size, shape and density of the dissolving entity and hence its location and/or distribution within the vessel, very different dissolution results may be obtained. Several authors have attempted to establish a relationship between stirring and in vivo hydrodynamics (e.g. Scholz et al., 2002) but results are, as might be expected, inconsistent. For example, Scholz et al. were able to establish a correlation between a paddle method at $75 \mathrm{rpm}$ and the absorption of coarse or micronized felodipine in Labrador dogs in the fasted state (Scholz et al., 2003). In other studies (Ishii et al., 1996), the authors suggested that, in order to predict behavior in Beagle dogs, the optimal paddle speed for ibuprofen capsules would be $56 \mathrm{rpm}$. Yet other studies indicated that a paddle speed of $30 \mathrm{rpm}$ would achieve the best IVIVC for paracetamol tablets (Rostami-Hodjegan et al., 2002b). Other attempts to model gastric forces by correlating in vitro dissolution at various stirring rates in a USP apparatus 1 and 2 and in vivo bioavailability data have been successful in individual cases (Abrahamsson et al., 1998; Shameem et al., 1995). However, it is obviously not possible to generalize these findings since the hydrodynamic effects in the in vitro test and possibly also within the GI tract may be product specific. So, while predictive in vivo conditions may be established for individual products based on empirical IVIVCs, a more general in vivo relevant set of in vitro tests is not envisaged with this approach.

Another major issue resulting from variable hydrodynamics, especially for the (most commonly) used USP 2 apparatus, is the coning effect (Figure 2) that can occur at the bottom of the vessels (e.g. Qiu et al., 2009). Granules or particles with sufficiently high density form a mound, inhibiting dissolution in the stagnant zone below the paddle. Similar effects may 
occur in the rotating basket for particles small enough to pass through the basket mesh. This problem is frequently encountered during dissolution method development and, if not adequately addressed, has the potential to generate misleading in vitro data during drug product development. For example, in a study at AstraZeneca two different MR pellets formulations gave different in vitro profiles in a USP 2 method while they were bioequivalent in a human study. Coning was visually observed for the slower releasing formulation; when the dissolution study was repeated using a peak vessel, no difference between the two formulations could be demonstrated (personal communication). This example illustrates that coning effects observed in vitro are unlikely indicative of a similar phenomenon in vivo.

Coning is also mentioned in the USP general chapter on dissolution (US Pharmacopoeia, 2011), which states that the problem can be overcome by increasing the stirring speed to 75 or $100 \mathrm{rpm}$ in the paddle apparatus, or by replacing the round bottom dissolution vessels with socalled peak vessels. These strategies have recently been explored by Mirza et al.; they showed that the dissolution rate was greater in the peak vessel compared to the USP vessel (Mirza et al., 2013). Computational fluid dynamic assessment has indicated that shear heterogeneity in the regions where tablets are most likely to localize during dissolution testing is reduced using the peak vessel, but it was hypothesised that the higher shear rates might result in the inability to discriminate between true differences in dissolution rates (Baxter et al., 2005b). Coning may also be reduced by simply increasing the paddle speed during the dissolution test. Indeed, the dissolution rate is often increased at higher stirring rates as shown in the example illustrated by $\mathrm{Wu}$ et al., noting that if the stirring is too rapid, discrimination between different IR formulations is compromised (Wu et al., 2004). In an in vitro study using a limited number of model IR tablets (Shah et al., 1992), no significant increase in dissolution rate was noted with an increase in agitation rate from 75 to $100 \mathrm{rpm}$. It was concluded that the higher agitation rate of $100 \mathrm{rpm}$ is not necessary, especially in the context of QC.

There are additional pragmatic factors that need to be considered when using the USP 1 and 2 methods to minimize variability due to hydrodynamic effects. These have been well described elsewhere (Gray et al., 2009).

\subsubsection{Previous use in development}

USP 1 and 2 methods are most commonly used, mainly because dissolution testing has for decades been handled by departments/scientists associated with QC. These methods are in many respects robust and practical to handle and, if a single apparatus is employed for all 
development programs within a company, different formulations can easily be compared. During recent years, there has been an increased use of biorelevant media rather than pharmacopeial buffers in these methods. In particular, the paddle apparatus has been frequently used for BCS class II drugs in IR dosage forms in conjunction with biorelevant media to predict/describe food and formulation effects. A couple of representative examples include the prediction of food effects of danazol (Galia et al., 1998) and a comparison of various albendazole formulations (Galia et al., 1999).

The use of USP 1 and 2 apparatus is also prescribed for biowaiver testing, i.e. when in vivo bioequivalence studies are replaced by in vitro dissolution studies for rapidly dissolving IR tablets of BCS class I and III drugs (European Medical Agency, 2010). The use of these is made with the underlying assumption that in vivo relevant results will be obtained for such highly soluble drugs given that testing is performed under different $\mathrm{pH}$ covering the range in the GI tract. This has so far proven to be a successful approach and in many cases the biowaiver dissolution methods are able to predict the outcome of in vivo bioequivalence studies in healthy volunteers (Polli, 2008). It may even be possible to further extend the usage of in vitro dissolution testing for biowaiver assessment by considering biorelevant media in conjunction with additional validation with clinical data.

\subsection{Reciprocating Cylinder}

The reciprocating cylinder apparatus (Type 3) (Figure 1) was introduced much later into the Pharmacopeia (1991) and has yet to establish itself as a QC method, with only a few USP individual monographs recommending this apparatus. That said, the reciprocating cylinder apparatus offers some attractive features, especially in terms of assessing performance of dosage forms with MR characteristics. The apparatus is modeled on the concept of the disintegration tester, with the dosage form placed in an open cylinder fitted with a sieve at the bottom end and optionally also at the top end. This cylinder is placed in a vessel maintained at $37^{\circ} \mathrm{C}$ in a water bath, and the cylinder is moved up and down through the medium. The difference from the disintegration tester lies in two modifications. First, each dosage unit/cylinder is placed in a separate vessel, enabling release to be studied for each individual dosage unit. Second, it is possible to move the cylinder from one vessel to the next across a series of vessels. As each vessel can be filled with a different medium, this enables flexibility in the composition of the medium. In fact, with an astute choice of media, one can simulate movement of a non-disintegrating dosage form (e.g. controlled release tablet or pellets) 
through the entire gastrointestinal tract. So, particularly in cases where the dosage form will encounter a range of conditions before disintegrating/moving out of the gastrointestinal tract, the reciprocating cylinder apparatus offers the possibility to characterize the complete release profile in just one experiment.

\subsubsection{Dosage form types}

In principle, the reciprocating cylinder can be used for a wide variety of oral dosage forms. However, since the operating volume per vessel is quite low (see next section) it may be difficult to generate sink conditions and therefore this type of equipment is not as widely applicable as the paddle or basket methods for QC of IR dosage forms. On the other hand, for development purposes, the low volumes may simulate the actual release conditions better than the volumes required for the standard paddle and basket experiments. The reciprocating cylinder has been used successfully to study release from lipid-filled capsules (Jantratid et al., 2008a). In this study a clear benefit of the reciprocating action in keeping the lipid material adequately dispersed in the dissolution medium in comparison to the paddle method was demonstrated. The reciprocating cylinder may also be used for studying the release characteristics from enteric coated (EC) products, since the change in medium can be achieved simply by moving the cylinder into the next vessel. A particular benefit for EC products coated with polymers dissolving at higher $\mathrm{pH}$ is that the possibility of premature release can be checked at $\mathrm{pHs}$ relevant to the upper small intestine as well as the stomach by using three (or more) rows of vessels, each with a different $\mathrm{pH}$. Examples of using the reciprocating cylinder method can be found in Klein et al. (Klein et al., 2005, 2008) and Jantratid et al. (Jantratid et al., 2009). Li et al. used a similar setup for acquiring release profiles of formulations with multiple $\mathrm{pH}$-sensitive coating layers ( $\mathrm{Li}$ et al., 2002).

Using the same approach of multiple rows of vessels to represent conditions in various parts of the gastrointestinal tract, the reciprocating cylinder method can of course also be implemented for MR dosage forms. Studies using this test design were reported by Ramos Pezzini and Gomez Ferraz (Ramos Pezzini and Gomes Ferraz, 2009), Khamanga and Walker (Khamanga and Walker, 2006) and Klein and Dressman (Klein and Dressman, 2006; Klein, 2009). Such methods using the reciprocating cylinder can have great relevance for in vivo and are attractive for $\mathrm{QbD}$ purposes but, as described previously, may need to be modified somewhat in order to make them viable in a QC testing paradigm.

\subsubsection{Media volumes}


The reciprocating cylinder method employs a volume of up to $250 \mathrm{~mL}$ per vessel and, depending on the number of vessels used, the total volume employed can be significantly greater (for example six vessels sequentially would result in a total volume of $1.5 \mathrm{~L}$ ). If sink conditions for dissolution is required, this can be achieved by changing the vessel more often thereby enabling some flexibility in the volumes used. The Type 3 apparatus does not however offer the complete flexibility in media volume that can be achieved with the flowthrough system.

\subsubsection{Hydrodynamics}

The hydrodynamic patterns in the Type 3 apparatus are generated by the reciprocating movement of the cylinder. The resistance to flow can be changed by altering the mesh size of the sieve attached to the bottom of the cylinder. Rohrs et al. demonstrated that there can be a relationship between the paddle and reciprocating cylinder hydrodynamics (Rohrs et al., 1995 ) and recommended dip rates of less than $10 / \mathrm{min}$ to correspond to a stirring rate of 50 rpm in the paddle apparatus or $100 \mathrm{rpm}$ in the basket apparatus. An advantage of the reciprocating cylinder over the paddle and basket methods is that there is no hydrodynamic dead zone to deal with, as long as the formulation remains in the reciprocating basket and undissolved material does not collect at the bottom of the vessel (outer beaker) and the formulation does not adhere to the side of the cylinder. Dip rates can be varied over a wide range (typically 5-40 dpm, although at the high end of the range this can lead to splashing of the medium over the edge of the vessel into the water bath), with studies usually employing rates in the 10 to $25 \mathrm{dpm}$ range. The mesh size of the sieve on the bottom of the cylinder can also be adjusted to further fine-tune the hydrodynamics - the smaller the sieve mesh size, the more resistance to fluid flow in and out of the cylinder. Of course, there is a practical limit to how wide the mesh pores can be for pellet formulations, as the sieve must prevent the pellets from falling through the sieve into the vessel and therefore not being available for transfer to the next row of vessels.

\subsubsection{Previous use in development}

There are several reports in the literature, additional to those mentioned above, where the authors have optimized conditions in the reciprocating cylinder method for a particular drug/dosage form combination; most of these have studied dosage forms with delayed or ER characteristics. For example, Li et al. (Li et al., 2002) used the reciprocating cylinder apparatus to optimize release conditions for a new colon-targeting system by employing a 
series of buffers with different pHs. On the other hand, Missaghi and Fassihi indicated that, for a swellable matrix system containing diphenhydramine, the hydrodynamics in the reciprocating cylinder were too aggressive, even at low dip rates (8 dpm) (Missaghi and Fassihi, 2005). A few years later, Fotaki et al. published data indicating that the paddle, reciprocating cylinder and flow-through methods can all be useful for testing various types of MR dosage forms (seven in total) (Fotaki et al., 2009); in this article, the authors also linked the results to the in vivo performance of the dosage form. Most recently, Klein et al. (Klein et al., 2013) were able to show that the reciprocating cylinder apparatus is very useful for creating individual in vitro profiles. The aim of the studies was to explain the variability of individual absorption profiles observed after fasted administration of a diclofenac ER pellet formulation. By accounting for the variability in gastric emptying of the pellets in the in vitro test design, they were able to establish a good correlation with the observed in vivo performance of the formulation. However, it is apparent that more work is still needed to establish useful parameter combinations (dip rate, sieve mesh, media, volume) to facilitate optimal use of the reciprocating cylinder method for the development of the various types of MR dosage forms.

\subsection{Flow-through Cell}

The flow-through cell is described as Apparatus 4 (Figure 1) (The United States Pharmacopeial Convention, Inc., 2013). The assembly, as described in the Pharmacopeias, consists of a reservoir containing the dissolution medium, a water bath that maintains the dissolution medium at $37 \pm 0.5^{\circ} \mathrm{C}$ and a pump that forces it upwards through the cell with a delivery range between 240 and $960 \mathrm{~mL}$ per hour using standard flow rates of 4, 8 and 16 $\mathrm{mL} / \mathrm{min}$ (The United States Pharmacopeial Convention, Inc., 2013). The flow-through cell is mounted vertically with a filter system that prevents the escape of undissolved particles from the top of the cell. The bottom cone of the cell is usually filled with small glass beads $(\sim 1$ $\mathrm{mm} \varnothing$ ) with one bead $(\sim 5 \mathrm{~mm} \varnothing)$ positioned at the apex to protect the fluid entry tube (The United States Pharmacopeial Convention, Inc., 2013). For orally administered solid dosage forms, two standard cells are used: the large cell (22.6 $\mathrm{mm}$ i.d) and the small cell (12 $\mathrm{mm}$ i.d). A tablet holder is usually used for positioning the dosage form (The United States Pharmacopeial Convention, Inc., 2013). The Apparatus 4 can operate as an open system with fresh dissolution medium from the reservoir continuously passing through the cell or as a closed system where a fixed volume of dissolution medium is recycled (Fotaki et al., 2005a). Although there is currently no performance test for dissolution procedures that rely on 
Apparatus 4 described in the Pharmacopeia, Eaton et al. evaluated salicylic acid tablets as a candidate reference material and proposed a performance verification test which proved to be capable of probing effects in several critical parameters of Apparatus 4 such as size of glass beads, cell temperature, flow rate and level of deaeration (Eaton et al., 2012).

Shiko et al. studied the hydrodynamics inside the flow-through cell using magnetic resonance imaging and emphasized the necessity to understand the influence of test conditions on dissolution behavior in defining robust flow-through dissolution methods (Figure 3) (Shiko et al., 2011). They established the conditions which offered more uniform flow profiles when using the wider cell, placing the tablet vertically and utilizing lower flow rates. It should be noted that removing the $1 \mathrm{~mm}$ beads and operating with only the $5 \mathrm{~mm}$ ruby bead in place yielded a chaotic and asymmetric flow field in the empty $12 \mathrm{~mm}$ i.d. USP 4 cell, even at the lowest flow rate of $4 \mathrm{~mL} / \mathrm{min}$. The use of $1 \mathrm{~mm}$ beads dampened the jet-like behavior and acted as a distributor of the flow although it did not suffice to ensure a fully developed laminar flow profile. However, the assignment of hydrodynamics to truly laminar or turbulent flow in the absence of $1 \mathrm{~mm}$ glass beads has been challenged by others (Kakhi, 2009). The choice of the appropriate filter may also become an issue when assessing the dissolution of microparticles or when it becomes evident that insoluble or sticky particles block the filter and create backpressure into the cell (Fotaki et al., 2005a).

When operating the type 4 apparatus as an open system, raw data (i.e. amounts dissolved during specific time intervals) are collected in non-cumulative form. Depending on the selected time interval, this can allow the estimation of release rates. Where the aim is to characterize the kinetics of the process, transformation of data to the cumulative form should be avoided as any mistakes associated with the estimation of the total drug released during a specific time interval will accumulate over subsequent time intervals and the fundamental assumption of independence of errors is violated (Vertzoni et al., 2006). Fang et al. described a biorelevant in vitro dissolution method using the non-cumulative form of data collected with Apparatus 4 that could be applied in the areas of formulation selection, lot-to-lot variability, and food effect, in order to predict in vivo drug performance in early phase formulation development (Fang et al., 2010). However, when data are used in their non-cumulative form, the estimated dissolution/release rates are heavily dependent on the duration of the time interval. As a result, when dissolution limits absorption, the cumulative data are often compared directly with the cumulative deconvoluted plasma profiles (Fotaki and Vertzoni, 2010; Fotaki et al., 2005b) 
Kalantzi et al. showed that dissolution of paracetamol from IR formulations using the flowthrough apparatus is rapid and complete in media simulating the fasted state whereas it is substantially delayed in the corresponding media simulating the fed state. These data reflected the delayed disintegration times of these tablets in the fed stomach (Kalantzi et al., 2005). In vitro experiments with modified biorelevant media in terms of bile salt and phospholipid levels for either fasted or fed conditions with the flow-through cell apparatus gave good IVIVCs for danazol capsules under both simulated conditions. In that study, a flow rate of 8 $\mathrm{mL} / \mathrm{min}$ was considered the most appropriate choice to simulate in vivo bioavailablity of danazol (Sunesen et al., 2005).

Apparatus 4 is most frequently used for examining the dissolution characteristics of MR dosage forms, as the single dosage form can be exposed to the different conditions across the gastrointestinal tract (Fotaki and Vertzoni, 2010), but it has also been used in the evaluation of solid dispersions (Thybo et al., 2008).

Data with regard to the superiority of Apparatus 4 over the other compendial apparatus are not in agreement. Okumu et al. showed superiority of Apparatus 4 over Apparatus 2 under constant $\mathrm{pH}$ conditions in the prediction of the oral absorption of montelukast sodium (filmcoated tablets) (Okumu et al., 2008). In another study, the importance of the hydrodynamics conditions of the USP Apparatus 2, 3, and 4 in the development of IVIVCs for monolithic dosage forms (a BCS class II compound housed in a hydrophilic matrix formulation and for a BCS class I compound housed in an osmotic pump formulation) was assessed. Even though in vitro hydrodynamics affected the release profile from the hydrophilic matrix in biorelevant dissolution experiments in the fasted state, all three apparatus were equally useful in predicting the actual in vivo profile on an average basis (Fotaki et al., 2009). Biorelevant dissolution testing with USP Apparatus 3 and 4 successfully led to the prediction of food effect for MR diclofenac sodium pellets with the Apparatus 4 slightly superior to the Apparatus 3. In this study, the disadvantage of compendial dissolution tests (phosphate buffer with USP Apparatus 1 and 2) for this kind of prediction was also demonstrated (Jantratid et al., 2009).

Based on these data, the usefulness of Apparatus 4 in relation to other dissolution apparatus in predicting intralumenal release/dissolution appears to require further evaluation. 


\section{Overcoming the limitations of pharmacopoeial setups - recent advances and current challenges}

In general, the pharmacopeia have provided the industry with reliable QC dissolution methods for various types of oral dosage forms. However, in terms of both the apparatus and the media used, these compendial systems are far from optimized for dosage form development and predictive evaluation. As they are designed for easy implementation in most laboratory settings while providing sink conditions for drug release, compendial media serve the purpose of QC well. . When the paradigm shifts from QC to development, it may no longer be appropriate to merely add enzymes (to deal with capsule shell dissolution) or synthetic surfactants (to deal with poorly soluble drugs) to plain aqueous buffer solutions. To bridge the gap between QC and development, it is particularly important to take the in vivo physiological conditions under which dissolution takes place into consideration. To achieve this aim, the importance of using biorelevant media was first proposed in 1998 (Dressman et al., 1998). Since then, there have been considerable efforts to characterize the luminal contents of the upper GI tract (Clarysse et al., 2009; Diakidou et al., 2009; Kalantzi et al., 2006; Lindahl et al., 1997; Perez de la Cruz Moreno et al., 2006) and several additional media have been proposed (Erceg et al., 2012; Jantratid et al., 2008b; Vertzoni et al., 2005, 2010). The upshot is that most development groups in the pharmaceutical industry presently use some form of biorelevant media in order to characterize new drug candidates and to screen formulations. In fact, the use is so widespread that instant versions of the media are now commercially available (Kloefer et al., 2010). It is also important to establish whether standard methods can be used for rapidly or very rapidly dissolving products of BCS class I and III drugs for in vivo predictions without prior IVIVC. Going forward, it will be important to continue characterizing the contents of the GI tract in various patient groups, age groups etc. to get a better idea of how dosage forms will perform under conditions of therapeutic use in these subpopulations.

In terms of apparatus, most dissolution tests are currently performed in conventional apparatus (such as the USP1/2) employing 'simple' experimental conditions (e.g. sink conditions using a single well-defined medium and volume at a constant $\mathrm{pH}$ ). These conditions are quite different from the in vivo situation, where GI transit exposes the drug/formulation to a rapidly changing and complex luminal environment. During transit, the drug may undergo dissolution, degradation, supersaturation, precipitation and re-dissolution; processes that may not all be evident using a conventional dissolution method. Therefore, in 
order to better predict the in vivo behavior, more physiologically relevant multi-compartment dissolution methods that address the changing GI environment should be considered.

\subsection{Predicting supersaturation and precipitation}

Contemporary drug pipelines are trending towards more and more poorly water soluble drugs requiring novel formulation strategies to provide for appropriate oral bioavailability. The precipitation of a supersaturated drug within the gastrointestinal tract can be an unwanted result following administration of the drug dosage form to the body. Not only does precipitation reduce the amount of drug that is in solution and available for absorption across the gut wall, but it may also lead to increased variability in bioavailability with downstream issues potentially impacting efficacy and safety.

Poorly water-soluble drugs may precipitate in vivo due to a number of factors related either to the physicochemical properties of the drug itself or due to the nature of the formulation used. For example, drugs may precipitate during the transit through the gastrointestinal tract due to step changes in $\mathrm{pH}$ in transitioning from the stomach to the intestine, through dilution of the formulation with body fluids or by means of digestion of solubilizing excipients which compose the formulation.

A strategy to overcome solubility limitations is to increase the apparent concentration of drug in the gastrointestinal lumen through supersaturation. Using this strategy, a greater amount of drug is present in solution for a longer time period such that more drug is available for absorption. However, in generating supersaturation, the drug in solution is thermodynamically unstable, constituting the driving force for precipitation. Supersaturation may be achieved using a number of different formulation approaches such as solid dispersions (Frank et al., 2012), crystalline salts (Guzmán et al., 2007), formulating with co-solvents (Carlert et al., 2010), adsorption-based formulations (Van Speybroeck et al., 2010), and lipid based formulations (Anby et al., 2012; Yeap et al., 2013).. For weak bases (irrespective of the formulation used) transfer from the acidic stomach to the more $\mathrm{pH}$ neutral small intestine can induce supersaturation and thus a metastable state (Kostewicz et al., 2004)

An assessment of drug supersaturation and precipitation and for poorly soluble drugs is vital during dosage formulation development and formulation screening. Given the complexity of supersaturation and precipitation in the GI lumen, there are a multitude of factors that need to be considered when evaluating these processes in vitro. For supersaturation, luminal 
concentrations can be influenced by gastric emptying, permeability, ionization characteristics of the API, solubilization by bile acid micelles and the dissolution characteristics of the formulation of interest. Further, precipitation may be influenced by the $\mathrm{pH}$ change observed between stomach and intestine, dilution of the formulation by GI luminal fluids, digestion of solubilizing excipients, the nature of excipients used in the formulation and/or the ambient composition of the luminal fluids (Bevernage et al., 2012a; Brouwers et al., 2009; Curatolo et al., 2009; Kostewicz et al., 2004; Lindfors et al., 2008; Sassene et al., 2010; Tønsberg et al., 2010; Van Speybroeck et al., 2010). Further, the composition of the GI luminal fluids is likely to vary considerably following the ingestion of food, which may further impact the supersaturation and precipitation characteristics of the formulation.

The current status of in vitro approaches to evaluate supersaturation and precipitation has recently been reviewed by Bevernage et al. (2012b). A number of case studies reported in literature illustrate some of the challenges that exist in designing appropriate tools to capture the complex supersaturation and precipitation behavior in a biorelevant fashion. In case of a weak base, the drug may dissolve completely at gastric $\mathrm{pH}$ but precipitate in the small intestine where the $\mathrm{pH}$ increases and solubility drops. Kostewicz et al. originally presented a transfer model in which a two compartment USP dissolution method is applied that simulates the stomach and intestine, respectively (Figure 4) (Kostewicz et al., 2004). In this experimental set-up, a drug solution is placed in a simulated gastric fluid compartment (donor phase), which is pumped into the simulated intestinal compartment (acceptor phase) at a constant rate within the range that can be observed for gastric emptying (in this case, flow rates between 0.5 and $9.0 \mathrm{~mL} / \mathrm{min}$ were used). Drug precipitation in the acceptor compartment was evaluated by analysis of the concentration versus time profile in the intestinal compartment. Through the application of this model, Kostewicz et al. was able to show that following transfer of the gastric compartment into the intestinal compartment, a significant degree of supersaturation was observed for each of the weak bases examined with precipitation occurring under conditions simulating the fasting but not fed states (Kostewicz et al., 2004). Interestingly, flow rates had an effect on the maximum concentration measured such that a faster transfer rate resulted in a higher concentration achieved prior to precipitation. These results suggested that gastric emptying rates could have an important impact on the precipitation kinetics. The results from this study showed that this transfer model could be used not only to examine supersaturation but also precipitation under various physiological conditions. 
To further understand the underlying in vitro and in vivo drug precipitation mechanisms, Arnold (Arnold et al., 2011) introduced online dynamic image analysis and inline disperse Raman spectroscopy to assess changes in the acceptor compartment of the transfer model and applied a power law modeling approach to propose a kinetic nucleation and growth paradigm. By including the in-line dynamic image analysis, the formation of the precipitate and subsequent aggregation could be monitored in real time. By examining the Raman spectroscopy as a function of time, Arnold et al. (Arnold et al., 2011) showed that this method was a useful tool for monitoring the fraction of drug precipitated, as an alternative to measuring the concentration of dissolved drug.

Carlert et al. utilized the transfer model and two additional in vitro methods to examine the intestinal precipitation characteristics of a BCS class II weak base (Carlert et al., 2010). The drug was formulated as a co-solvent solution. The precipitation of the drug solution was evaluated using either the transfer model set-up based on Kostewicz et al. (Kostewicz et al., 2004) or a scaled down version of the transfer model in which a concentrated FaSSIF solution was added to the intestinal compartment in order to ensure that the $\mathrm{pH}$ and bile salt concentration remained constant. To examine the influence of hydrodynamics on precipitation, a USP 2 mini-vessel at $150 \mathrm{rpm}$ was compared with a much gentler hydrodynamic condition. The weak base precipitated in each of the experimental conditions; however, the gentler hydrodynamic condition resulted in slower crystallization, illustrating the importance of hydrodynamics in precipitation prediction. Interestingly, results from a parallel in vivo study suggested that precipitation did not influence the PK profile. The absence of in vivo precipitation might be attributed to reduced hydrodynamic stress in the gut lumen and/or to the fact that the closed system used in each of the experimental methods does not take into account removal of drug from the intestinal fluid by absorption across the intestinal membrane. Since the drug is classified as a high permeability compound, it is likely that rapid absorption in vivo reduces luminal concentrations and, as a consequence, the driving force for precipitation. These results suggest that permeability needs to be considered when evaluating the in vitro precipitation characteristics of a highly permeable compound. To take into account the absorption of drug, the use of an absorptive sink in an in vitro method to examine supersaturation and precipitation is described in more detail in section 3.2.

Psachoulias et al., (Psachoulias et al., 2012) recently published a study in which a threecompartment in vitro apparatus (comprising of a stomach, intestinal and reservoir compartment) was used to predict concentrations of weakly basic drug that can be anticipated 
in the upper small intestine. Using this experimental design, dose dependent in vitro precipitation was demonstrated for one of the drugs whilst for the other drug, no precipitation was observed. Both observations were consistent with in vivo data. The results from this study suggest that the predictability of the in vitro model was increased by the use of a modified biorelevant medium (FaSSIF) containing both cholesterol and sodium oleate, which more closely reflects the conditions of the proximal small intestine. Further, the addition of concentrated biorelevant media (reservoir compartment) to the intestinal compartment, helped to maintain relevant bile salt and lecithin concentrations following dilution by the gastric medium. Finally, a first order gastric emptying rate was used, which is in better alignment with the kinetics of gastric emptying under fasting conditions (Moore et al., 1984). Incorporating all of these factors in the in vitro experimental design ensured conditions that are more consistent with the in vivo GI situation, resulting in a more accurate prediction of the concentrations that can be anticipated in vivo.

Psachoulias et al. (Psachoulias et al., 2012) were also one of the first to compare the solid state characteristics of precipitates formed in vitro and in vivo (collected from the gastrointestinal tract of healthy volunteers). In case of ketoconazole, differences were observed: in vitro the precipitate was crystalline; in vivo, the precipitate was amorphous. Whilst the impact of this difference is not known, the solid state characteristics of the precipitate may play an important role in terms of re-dissolution of the precipitate within the gut lumen, which can influence the ultimate rate and extent of absorption.

Although various in vitro assays have been described, their ability to predict in vivo supersaturation and precipitation can only really be evaluated when in vivo reference data is available. Not only is in vivo data necessary for providing an indication for supersaturation or precipitation but also to guide method optimization. Since fundamental parameters important to supersaturation and precipitation in vivo have not been fully characterized yet, methods can only be optimized by comparison to reference in vivo data for the drugs under investigation.

In the future, the results from the in vitro supersaturation and precipitation tests should not be evaluated in isolation; rather they should be incorporated with physiologically based pharmacokinetic modeling (PBPK). In the past, combination of in vitro dissolution data with PBPK modeling has led to more accurate predictions of plasma levels. It is expected that incorporation of supersaturation and precipitation data into PBPK models will improve this 
mechanistic approach to absorption modeling and help tease out the importance of these parameters on the overall absorption process.

\subsection{Accounting for absorption in formulation evaluation}

Compendial in vitro evaluation of strategies to overcome dissolution/solubility-related absorption issues focuses on the estimation of intraluminal concentrations by dissolution and precipitation assessment in setups that mostly ignore intestinal absorption. To predict drug flux and fraction absorbed, estimated concentrations are combined with drug permeability. However, ignoring absorption during dissolution and precipitation assessment may compromise the biorelevance and predictive power of in vitro formulation evaluation (Takano et al., 2012). Firstly, absorption of moderate to high permeability drugs creates a sink effect that may increase dissolution and decrease precipitation. Secondly, as pointed out in previous sections, various solubility- and dissolution-enhancing strategies aiming at increased intraluminal concentrations, may additionally affect drug permeability.

The impact of an absorptive sink on precipitation was recently demonstrated by Bevernage et al. using the lipophilic model compound loviride (Bevernage et al., 2012a). Upon supersaturation induction of loviride, precipitation was significantly reduced at the apical side of a Caco-2 monolayer compared to a closed, non-absorptive system. In addition, the effect of a precipitation inhibitor (HPMC) was overestimated in absence of an absorptive sink, illustrating the importance of considering absorption during the evaluation of supersaturating drug delivery systems. The absence of an absorptive sink in standard supersaturation assays has been suggested as an important cause of poor in vitro-in vivo correlations for supersaturating drug delivery systems (Bevernage et al., 2012a; Carlert et al., 2010).

Food or formulation-based strategies to enhance drug solubility, including micellar encapsulation (Miller et al., 2011; Yano et al., 2010), cyclodextrin complexation (Loftsson and Brewster, 2011) and even cosolvency (Beig et al., 2012), may reduce permeability. Conceptually, this can be considered the result of a decreased free fraction of the drug and/or a reduced partition coefficient of the drug between the intraluminal medium and the membrane (Beig et al., 2012; Miller and Dahan, 2012; Miller et al., 2011). Hence, care is required to understand the impact of increased solubility and consequently decreased permeability on formulation development. It should be noted that this solubility-permeability interplay may be further complicated by possible interactions between food digestion products 
or solubilizing excipients and intestinal transporters or enzymes (Fleisher et al., 1999; Pang et al., 2006).

\subsubsection{Assessment of permeation from relevant samples}

The simplest way to account for the solubility-permeability interplay is the assessment of permeability from relevant samples, i.e. originating from solubility or dissolution experiments, preferably in biorelevant media. Examples include the first attempt to integrate dissolution with Caco-2 permeation (Ginski and Polli, 1999) and permeability determination across rat intestinal tissue upon in vitro lipolysis of lipid-based formulations (Dahan and Hoffman, 2007). To assess drug permeation from real human intestinal fluids (HIF), duodenal aspirates collected after administration of dosage forms to healthy volunteers have been applied on the apical side of Caco-2 monolayers. Vertzoni et al. demonstrated the solubilitypermeability interplay from real HIF samples, aspirated after administration of the lipophilic drug danazol together with a meal (Vertzoni et al., 2012). Danazol solubilisation by lipid digestion products significantly reduced permeability; however, this effect was overcompensated by the increased danazol concentrations, overall resulting in an enhanced flux. In a similar experiment with HIF samples aspirated upon oral intake of a solubilizing amprenavir formulation (Agenerase $\AA$ ), Brouwers et al. revealed the multifactorial effect of the surfactant $\mathrm{d}$ - $\alpha$-tocopheryl polyethyleneglycol 1000 succinate (TPGS) on amprenavir absorption: increased amprenavir concentrations by solubilisation, entrapment of amprenavir in TPGS-based micelles (reduced permeability), and inhibition of the efflux carrier P-gp (increased permeability) (Brouwers et al., 2006).

Although permeability assessment from relevant samples does not directly integrate absorption into dissolution testing (implying that absorptive sink effects are not simulated) it offers a relatively straightforward way to identify permeability issues upon solubilisation.

\subsubsection{Integrating absorption in in vitro dissolution and precipitation setups}

The in vitro simulation of the absorptive sink present in vivo requires modification of classic dissolution setups. Various approaches have been reported in literature, employing different implementations of permeation (e.g. separate absorption module versus direct integration in the dissolution module, Caco-2 monolayers versus less biorelevant approaches).

\subsubsection{Separate absorption module}


Kobayashi et al. developed a transfer model consisting of a gastric and duodenal vessel and a separate side-by-side diffusion cell with a Caco-2 monolayer mounted between donor and acceptor side (Kobayashi et al., 2001). Peristaltic pumps ensured continuous transfer between the different modules. The system has been used to simultaneously monitor dissolution and permeation for the poorly soluble drugs albendazole and dipyridamole from different formulations (Sugawara et al., 2005). Motz et al. developed a similar system, using the compendial flow through dissolution cell (USP 4) coupled to a Caco-2 permeation module by means of a stream splitter (Motz et al., 2007). The apparatus has been validated using several formulations of propranolol $\mathrm{HCl}$ but its added value for more challenging drugs has not been reported.

To avoid the use of Caco-2 cells, which may compromise routine use, Gu et al. attempted to simulate the absorptive sink in a multi-vessel setup by the continuous transfer of dissolved drug from the intestinal compartment to the 'absorption' compartment, separated using a 0.22 $\mu \mathrm{m}$ filter ( $\mathrm{Gu}$ et al., 2005). By adjusting the flow rate between these two compartments, various permeability values can be simulated. The system was applied to evaluate precipitation and 'permeability' of two poorly soluble weak bases (dipyridamole and cinnarizine) upon transfer from the gastric to the intestinal compartment. Since both dipyridamole and cinnarizine are lipophilic drugs exhibiting moderate to high permeability, a high flow rate between the intestinal and absorption compartments was used (corresponding to an absorption rate constant of $0.01 \mathrm{~min}^{-1}$ ). Implementing the absorptive sink allowed to investigate precipitation in more biorelevant conditions.

It should be noted that also the TNO gastroIntestinal Model (TIM) includes special dialysis or filtration membrane systems, connected to the jejunum and ileum compartments. These modules enable the assessment of the bioaccessible fraction, i.e. the fraction of drug potentially available for small intestinal absorption (Souliman et al., 2006, 2007). In combination with intestinal absorption models (e.g. Caco-2 cells), TIM gives relevant information on bioavailability (Déat et al., 2009; Haraldsson et al., 2005; Verwei et al., 2006). The TIM system will be further discussed in Section 3.5.

\subsubsection{Direct integration of absorption in the dissolution module}

In the above-mentioned systems with a separate 'absorption' module, one may adjust the strength of the absorptive sink effect by altering the flow rate between the dissolution and absorption modules. While this can be interesting for mechanistic studies, it may be difficult 
to set a biorelevant sink, depending on the mucosal permeability for the drug. Direct dissolution and/or precipitation at the apical side of a Caco-2 cell monolayer allows integration of a more biorelevant absorptive sink. Mellaerts et al. applied this approach to demonstrate enhanced itraconazole permeation following application of a supersaturating dosage form (ordered mesoporous silica) in a standard Caco-2 dual chamber, whether or not preceded by an acidic dissolution step (Mellaerts et al., 2008).

Kataoka et al. optimized a side-by-side dual chamber system to allow for dissolution of solid forms at the apical side of a Caco-2 cell monolayer (Kataoka et al., 2012). Simulated intestinal fluid (FaSSIF) at pH 6.5 was used as the apical medium $(8 \mathrm{~mL})$, while isotonic buffer ( $\mathrm{pH}$ 7.4) with serum albumin was used as basal medium (5.5 mL) to ensure sink conditions. Both compartments were stirred at $200 \mathrm{rpm}$. In this so-called dissolution/permeation (D/P) system, the absorptive sink is directly determined by the Caco-2 permeability for the drug. In addition, the permeated amount accounts for formulation-related effects on apical concentrations as well as on permeability. The D/P system is designed to allow direct in vitro-in vivo correlations by enabling the use of clinically relevant (scaleddown) doses. As such, a correlation between the human fraction absorbed and the permeated amount in the $\mathrm{D} / \mathrm{P}$ system has been established for poorly water soluble reference drugs (Kataoka et al., 2003).

The D/P system has further been used to predict the net food effect (i.e. balance of solubility/dissolution improvement and permeability reduction) on the absorption of poorly water soluble drugs (Kataoka et al., 2006). For this purpose, dissolution and permeation from solid forms was compared between FaSSIF and FeSSIF. The standard FeSSIF composition was modified ( $\mathrm{pH} 6.5$ and decreased osmolality) to ensure compatibility with the Caco-2 monolayer. It should be noted that more complex intestinal media, e.g. containing lipid digestion products, are currently not compatible with the D/P system. Despite this limitation, the D/P system was able to predict in vivo food effects for albendazole and danazol.

The D/P system has been applied to rank order different solid dosage forms (solid dispersion, nano- and microsized) of fenofibrate and predict formulation performance in rats (Buch et al., 2009). Similarly, Kataoka et al. demonstrated the usefulness of the D/P system for evaluation of solubilizing and supersaturating formulations of danazol (low solubility, high permeability) and pranlukast (low solubility, low permeability) (Kataoka et al., 2011). Compared to a suspension, the enabling formulations increased apical concentrations of both drugs in the D/P 
system; however, the permeated amount was only improved for danazol. This observation was in agreement with an increase in rat bioavailability for danazol but not for pranlukast, illustrating the importance of simultaneously assessing dissolution, precipitation and permeation when evaluating absorption-enhancing strategies.

Finally, the use of the Caco-2 monolayer enables the D/P system to capture the effects of intestinal transporters and to account for transporter-related drug-drug, drug-food, and drugexcipient interactions. For instance, using the D/P system, Kataoka et al. predicted the effect of co-administering an inhibitor of the efflux carrier P-gp (erythromycin) on the absorption of the P-gp substrates fexofenadine and talinolol (Kataoka et al., 2011). In addition, the authors demonstrated the double effect of the surfactant Cremophor EL® on saquinavir absorption: the permeated amount of saquinavir in the D/P was increased as a result of both solubility/dissolution enhancement and inhibition of P-gp mediated efflux.

\subsubsection{Biphasic dissolution tests}

While interesting from a biorelevance perspective, the use of Caco-2 cells in the D/P system also implies some disadvantages, including reduced throughput, limited size (only formulation intermediates and not final dosage forms can be investigated), non-compendial hydrodynamics, and compatibility issues between dissolution media and monolayer integrity. Biphasic dissolution tests, in which the absorptive sink is created by an organic solvent that is immiscible with the (biorelevant) dissolution medium, may provide an alternative. Shi et al. employed such a biphasic system to evaluate the release of the poorly soluble drug celecoxib from three formulations (the commercial Celebrex® capsule, a solution containing co-solvent and surfactant and a supersaturable self-emulsifying drug delivery system (S-SEDDS)) (Shi et al., 2010). The biphasic system, consisting of a USP 4 apparatus for aqueous dissolution under non-sink conditions connected to a USP 2 apparatus containing an additional octanol layer to create an absorptive sink, enabled discrimination among the three formulations and prediction of in vivo celecoxib bioavailability. Interestingly, release profiles in monophasic systems under both sink and non-sink conditions did not predict the in vivo formulation performance, demonstrating the added value of including an absorptive sink in the evaluation of enabling formulations.

Obviously, biorelevance issues are the major downside of creating an absorptive sink by means of a biphasic system. For instance, it is unclear to what extent direct contact between the drug and the organic solvent affects dissolution in a non-relevant way. In addition, the 
absorptive sink is solely dependent on the drug's partition coefficient between the dissolution medium and the organic solvent and, consequently, only slightly related to the membrane permeability. Finally, the biphasic system cannot account for drug-drug, drug-food or drugexcipient interactions at the level of intestinal transporters or enzymes.

Without doubt, integrating absorption into in vitro formulation evaluation is critical for in vivo performance prediction of certain absorption-enabling strategies for poorly water soluble drugs. A variety of non-compendial and non-standardized systems has been presented in literature; however, their added value is not always clearly demonstrated and validation is often anecdotal. Systematic and comprehensive research is needed to establish which models (simplicity versus biorelevance) significantly improve performance prediction of specific absorption-enabling scenarios.

\subsection{Mimicking the impact of gut motility}

\subsubsection{Importance of hydrodynamics for drug release and dissolution}

Currently, the characterisation of drug release from oral drug delivery systems is usually performed using compendial apparatus which use physical stresses on the delivery system that have not been validated against the actual physiological conditions prevailing along the gastrointestinal tract. At the time of development of the compendial dissolution and disintegration test methods, the knowledge on gastrointestinal motility and resulting parameters like transit times of dosage forms through the different regions of the gastrointestinal tract, mechanical stresses like pressure and acceleration, media flow (hydrodynamics), shear stress and media contact, was very limited. However, due to the development of modern non-invasive diagnostic techniques, this knowledge has significantly increased over the last two decades. During that time, it has also become obvious that such motility related parameters might significantly influence drug release behaviour and drug absorption. There are several examples where GI motility can directly influence drug absorption. One such example is in the case of accidental dose dumping which was observed for theophylline ER products (Hendeles et al., 1984) and also for diclofenac ER tablets, for which late or irregular plasma peaks were observed (Figure 5). Loss of absorption may also occur as a result of transfer out of the absorptive area (absorption window) (Weitschies et al., 2008). Furthermore, hydrodynamic conditions will influence disintegration of IR dosage forms that can be of importance if the disintegration is rate-limiting for absorption. For example, this has been shown for BCS class III drugs given together with food in which case 
meal viscosity resulted in a slower tablet disintegration (Radwan et al., 2012). Another example is the difference in onset of absorption of ibuprofen caused by differences in the disintegration of capsule shells of various quality (Cole et al., 2004). Hydrodynamics will also influence drug particle dissolution through effects on the unstirred water layer around dissolving particles. This has proved to be significant in vivo, especially for larger drug particles (Sheng et al., 2008; Scholz et al., 2003). Also in the case of supersaturated drug solutions, stirring conditions are critical for nucleation and particle growth in the drug precipitation process (Carlert et al., 2010).

\subsubsection{Attempts to model the impact of GI motility in dissolution testing}

There are three types of challenges in modelling motility effects in dissolution testing. Firstly, the understanding and characterisation of GI hydrodynamics, in a way that is meaningful to pharmaceutical dissolution testing, has been limited. However as described previously, better insights in terms of GI hydrodynamics have been gained in recent years. Secondly, the in vivo hydrodynamics conditions are very heterogeneous and vary from the resting conditions in fundus to the jet-like propulsions close to the pylorus in the fed stomach and also strong pressure forces at the ileocaecal valve during the gastro-ileocecal reflex. It is clearly not realistic to capture all these circumstances in one relatively simple model. Different approaches and their merits will be briefly addressed below. Finally, the hydrodynamic conditions provided in standard dissolution methods (including USP1 and 2) do not only lack in vivo relevance, they are also very heterogeneous and unpredictable because of the dependence on the location of the formulation in the dissolution test device as discussed before.

An overview of the hydrodynamic aspects intended to simulate physiological conditions for dissolution tests has recently been published by McAllister (McAllister, 2010). Generally, dissolution and disintegration methods and equipment have been developed over the last few decades to take into account the physiological conditions along the GI tract. However, they differ greatly with respect to design, complexity and to which extent mechanical conditions are represented. An overview of the most commonly used devices is given in Table 2.

Whilst the paddle-bead method (Aoki et al., 1992, 1993), rotating beaker (Abrahamsson et al., 2005) and stress test device (Figure 6) (Garbacz et al., 2008, 2009, 2010) can be considered as static systems trying to capture certain specific aspects of the in vivo hydrodynamics 
conditions, the artificial stomach-duodenal model (ASD) (Carino et al., 2006; Vatier et al., 1994), TIM-1 system (Minekus et al., 1995; Blanquet et al., 2004; Brouwers et al., 2011; Verwei et al., 2003) and the dynamic gastric model (DGM) (Vardakou et al., 2011a, 2011b; Wickham et al., 2009) are dynamic multi-compartmental simulators intended to mimic the main mechanical and chemical functions of the gastrointestinal tract. In the case of the DGM, this is limited to the stomach. The TIM system simulates both the stomach and small intestine (TIM-1) which can be extended to the whole GI tract with the large intestinal model (TIM-2) (Minekus et al., 1999; Tenjarla et al., 2007).

To date, none of the available test devices have the capability to simulate all aspects of the complex and highly dynamic situation that is present along the GI tract. However, TIM-1 and DGM systems already have the capability to simulate the kinetic functionality of the gut, or at least the stomach (DGM), in a very advanced way. This may offer the possibility to generate predictive data on the bioavailability of new compounds (Dickinson et al., 2012), as described in more detail in section 3.5. The complete TIM system does not need to be used for all experiments. In cases where the focus is just on the stomach parameters, only the gastric compartment has to be used; this avoids unnecessarily complex experiments. The more simple and static devices offer the possibility to mimic discrete conditions in separate experiments. In addition, a single experimental setup may be used to define a set of consecutive experiments where biorelevant parameters such as pressure or shear stress are applied and the susceptibility of a specific formulation towards such parameters can be more easily identified. The main disadvantage compared to complex whole gut simulation systems is the limitation to formulation testing. A typical application of such static devices for the simulation of biorelevant stress conditions might be to identify how likely an ER formulation is to show unwanted high release (dose-dumping) properties under physiological stress conditions, as for example during a high pressure event such as gastric emptying or passage through the ileocecal valve. Such an in vitro approach for the exclusion of dose dumping during the development phase using appropriate test systems, where we sufficiently understand the gastrointestinal processes involved, seems much more promising and relevant than in vivo PK tests under standard conditions since these are not intended to reflect a worst case scenario under patient-relevant conditions. Furthermore, it needs to be considered that unwanted high plasma peaks in pharmacokinetic fed state studies cannot be automatically regarded as a consequence of dose dumping from an ER delivery system; they may also result from the 
accumulation of released drug on top of food in the gastric fundus, as demonstrated for felodipine ER hydrogel matrix tablets (Weitschies et al., 2005).

In summary, the novel in vitro methods developed during recent years based on increased understanding of physiological and computational fluid dynamics hold some promise for improved in vivo predictions. However, evaluation and validation is so far merely anecdotal. Lack of systematic validation is one of the main limitations for a rational industrial use of dissolution methods aiming to capture hydrodynamics aspects. Such validations could provide the basis for defining a test strategy employing an array of methods covering different "worst case" stress situations as well as methods providing in vivo relevant "average" behaviour over the time span of the dissolution process. Additional validation work could also provide a basis for refinement of current tools or defining the need for novel approaches. Validation of bespoke methods should also include standard dissolution testing methods in order to show their superiority over standard methods.

\subsection{Accounting for digestion processes}

Digestion in the GI tract is an important factor to consider when evaluating the performance of dosage forms containing digestible excipients. Dosage forms with digestible excipients include for example lipid-based drug delivery systems containing tri- and diacylglycerols (TG \& DG); it should also be remembered that many surfactants are substrate for lipases (e.g. Christiansen et al., 2010; Fernandez et al., 2007; Li and McClements, 2011; Mohsin, 2012; Wulff-Pérez et al., 2012). In addition to this, an in vitro model simulating the digestion process is also relevant to investigate and understand potential drug-nutrient interactions, which is especially relevant for poorly soluble drugs.

\subsubsection{Gastric lipolysis}

In humans, lipid digestion in the stomach occurs through the secretion of gastric lipase (HGL) from the chief cells in the fundic mucosa of the stomach. After intake of a meal, HGL is responsible for 10-40\% of TG digestion (Armand et al., 1994; Carriere et al., 1993; Miled et al., 2000). HGL has a pH optimum between 5-6, but has been shown to have activity down to around pH 2 (Nury et al., 1987). The enzyme is primarily specific for the sn-3 position of the TG molecule and has a higher activity towards medium chain TG (MCT), than to long chain TG (LCT) (Gargouri et al., 1986). 
An inherent problem encountered when considering the development of a model simulating gastric digestion, is the lack of commercially available human gastric lipase. Most in vitro models use microbial lipases (Mercuri et al., 2011), but the specificity of these enzymes is not the same compared with the human gastric lipase (HGL); therefore they do not simulate the actual hydrolytic events in the stomach. Gastric lipase from dogs (DGL) has been used in the past, but is currently not available. Further, DGL is not an ideal substitute for HGL, as it has higher affinity for long chain (LC) lipids than for medium chain (MC) lipids, which is in contrast to HGL. Given these differences, it can be anticipated that it will not show the same hydrolytic profile as HGL.

In vitro models simulating gastric digestion encompass more complex models like the dynamic TNO gastro-Intestinal Model (TIM-1) and the Dynamic Gastric Model (DGM). These in vitro models also simulate other relevant aspects of digestion such as gastric hydrodynamics (DGM; Vardakou et al., 2011a) and peristalsis of the upper GI tract (TIM-1) and intestinal digestion to investigate intestinal bioaccessibility of compounds in various types of formulations in absence or presence of food (Blanquet et al., 2004; Minekus et al., 1995).

Simpler models, primarily focusing on gastric digestion have also been developed. Fernandez et al. developed a gastric digestion model using DGL (Fernandez et al., 2009) and showed that both Gelucire 44/14 and Labrasol are hydrolysed by DGL. However, as mentioned above, the specificity of DGL differs from that of HGL; furthermore, the model employed a rather high level of bile salts, which may have influenced the degree of hydrolysis. In summary, no reliable model of gastric lipolysis has so far been developed. Thus the impact of gastric lipolysis on performance of lipid based drug delivery systems is still not fully understood.

\subsubsection{Intestinal lipolysis}

Different one-compartment in vitro lipolysis models simulating the digestion in the duodenum and small intestine have been described and recently reviewed by Thomas and coworkers (Thomas et al., 2012b). These models are often used to simulate the digestion of lipid based drug delivery systems, and the impact this has on the solubilization of the drug dissolved in the delivery system. Although digestion is biochemically different in the stomach and small intestine, most studies using the in vitro lipolysis model thus far have only focused on the intestinal step (Thomas et al., 2012b). Increased focus on the importance of the gastric step and future generation of a commercially available human gastric lipase may change this picture. 
Intestinal in vitro lipolysis models typically employ porcine pancreatic extract as the enzyme source. As human pancreatic lipase (HPL), as well as porcine pancreatic lipase, is specific for the sn-1 and 3 position in a TG molecule, this seems like a reasonable surrogate, though differences in activity may still exist between the two enzymes towards pharmaceutical excipients (Fernandez et al., 2007). Besides pancreatic lipase, porcine pancreatic extract also contains other enzymes that can be relevant for lipid and surfactant digestion, such as carboxyl ester hydrolase $(\mathrm{CEH})$, pancreatic lipase-related protein 2 (PLRP2) and Phospholipase A2. However, these are not present in standardized amounts (Fernandez et al., 2007). Therefore, it might be advisable to employ the pure enzyme in order to study the functionality of these enzymes.

In vitro intestinal digestion typically takes place in a $\mathrm{pH}$-stat (Figure 7), keeping the $\mathrm{pH}$ constant at either 6.5 or 7 (Kaukonen et al., 2004; Zangenberg et al., 2001). The lipid-based formulations and the desired bile salt level are mixed at $37{ }^{\circ} \mathrm{C}$ and the pancreatic extract is added to initiate the lipolysis. The lipolysis is followed by titration of the formed free fatty acids with $\mathrm{NaOH}$. Calcium plays an important role in lipolysis: it removes the free fatty acids that otherwise would inhibit lipolysis, by blocking the surface of the emulsion particles. Calcium can either be added at a fixed level, or be added continuously during lipolysis. During lipolysis, samples are taken to assess solubilized drug, as well as precipitated drug in order to understand potential effects of the lipolysis on drug disposition. The experimental settings and the impact on the lipolysis experiment have been the subject of a number of investigations, but the relative link to the in vivo situation is much less explored. A number of publications have recently been published to define a standard protocol for the in vitro lipolysis (Williams et al., 2012a, 2012b).

Recently, it has been shown that some drugs precipitate in the amorphous form during in vitro digestion, which results in a fast redissolution once the drug is no longer in an environment of saturation (Larsen et al., 2011). The in vitro lipolysis model lacks the absorption step; however, these findings seem to correlate to in vivo observations (Thomas et al., 2012a). Solid state investigation of the pellet phase has therefore become more widespread when conducting in vitro lipolysis studies.

Only a limited number of publications exists describing IVIVC for in vitro lipolysis models; however, generally level C correlations (Malinowski et al., 1997) are obtained potentially predicting the rank order of the obtained area under the curve (AUC) for the evaluated 
formulations (e.g. Cuiné et al., 2007, 2008; Fatouros et al., 2008; Larsen et al., 2008; Porter et al., 2004). The in vitro lipolysis models have proven their potential value a number of times, though some room for improvement still exists.

\subsection{Dynamic multi-compartmental "GI tract in the lab" systems}

The challenges of simulating the dynamic environment of the upper GI tract are well recognised and a number of systems have been developed in an attempt to provide a more physiologically accurate simulation of in vivo dissolution. In contrast to other simpler and static set-ups, these systems attempt to faithfully capture all of the luminal processes that are involved in drug dissolution during gastrointestinal passage. This may offer several advantages over simpler systems:

1. The results generated are based on realistic human conditions and thus require less interpretation;

2. Unexpected interactions between different processes that may affect product performance are inherently investigated;

3. The systems can be controlled to mimic specific conditions and study effects of individual parameters, allowing mechanistical studies to be performed.

However these perceived advantages may lead to some sub-optimal use:

1. The identification of critical factors affecting product performance requires careful experimental design and so these systems are best suited to confirming rather than identifying an effect (Sheiner, 1997);

2. The complexity of the systems and perceived physiological similarity to the human gastrointestinal tract can lead to assumption-rich conclusions about the results of product performance and how this relates to clinical performance;

3. The complexity of the system design also increases the risk for artefactual results and variability in performance that is difficult to handle.

Even the most complex model is a simplification of a more complex reality. Therefore, it is important to understand how the model varies from the real situation and thus where interpretation of the results is required to allow extrapolation to the real situation. The following section discusses the complex and dynamic systems and identifies their usage to support product development; similarities and differences with the human GI tract; and differences and similarities between each model (summarized in Tables 1 and 2). Finally, 
further developments are identified that would make the models even more similar to the real situation.

\subsubsection{Artificial stomach duodenal model}

In terms of complexity, the simplest such adaptation beyond compendial dissolution apparatus is the artificial stomach duodenal model (ASD). This two compartment model comprises a gastric compartment linked to a second intestinal compartment intended to simulate the duodenal area. After dispersion of the drug or formulation in the gastric compartment, contents (liquids) are pumped at a controlled rate to the duodenal compartment. Mixing with simulated intestinal fluid (SIF) allows the dynamic processes of dissolution, precipitation, recrystallization and re-dissolution to be followed (Carino et al., 2006; Castela-Papin et al., 1999; Vatier et al., 1998). In addition to fluid transfer, the ASD is also configured to allow the infusion of fresh simulated GI fluids to each compartment. This combination of fluid transport and dilution causes a continuous variation in the concentration of drug substance in both gastric and duodenal compartments. The ASD has been used to aid formulation development and guide both salt and solid form selection (Bhattachar et al., 2011; Carino et al., 2006, 2010; Polster et al., 2010). However, the design of the ASD has some limitations. Table 1 shows the capabilities of the ASD relative to the more sophisticated models of the upper GI tract. The hydrodynamic conditions generated in the gastric compartment by the simple stirrer bar mechanism will be limiting in terms of replicating in vivo dosage form disintegration and initial dissolution. Additionally, whilst the continuous infusion of fresh simulated intestinal fluids in the duodenal compartment provides an increase in the effective volume for dissolution, the model lacks removal of compounds under sink conditions. This limitation may be particularly important for poorly soluble, highly permeable compounds for which the concentration gradient driving in vivo dissolution is maintained by permeation across the epithelial barrier. A further limitation of the ASD model is the absence of any control over digestive processes.

It is clear that, in order to address the limitations of simple dynamic systems like the ASD model a step-change in terms of equipment complexity is required. To replicate specific prandial conditions, it is necessary to provide control over physiological parameters such as temperature, $\mathrm{pH}$, peristaltic mixing and transit, gastric secretion (lipase, pepsin, $\mathrm{HCl}$ ) and small intestinal secretion (pancreatic juice, bile and sodium bicarbonate). 
A number of more complex GI-like simulators, offering control of these physiological parameters, have been reported in the literature including the TNO gastro-Intestinal Model (TIM) (Minekus et al., 1995, Minekus et al., 1999), the ModelGut / Dynamic Gastric Model (DGM) (Wickham et al., 2012) and, most recently, the Human Gastric Simulator [HGM] (Kong and Singh, 2010; Roman et al., 2012), all of which have originated from research in the nutrional/food science sector. Given their focus on the accurate simulation of digestive processes it is not surprising that the two more mature systems, the TIM-1 and DGM have found application in assessing the dissolution of pharmaceutical dosage forms.

\subsubsection{Dynamic Gastric Model}

The Modelgut (Institute for Food Research, Norwich, UK) Dynamic Gastric Model (DGM) was developed from insights gained from echo-planar magnetic resonance imaging studies on the gastric processing of complex meals (Marciani et al., 2000, 2001a, 2001b, 2004). The DGM is claimed to provide an accurate in vitro simulation of gastric mixing (including digestive addition around the gastric bolus), shear rates and forces, peristalsis and gastric emptying (Wickham et al., 2009). To date, a limited number of pharmaceutical applications of the DGM have been reported in the literature. One study which explored the ability of the DGM to replicate the dynamic digestion of a self-emulsifying drug delivery system [SEDDS] suggested that the DGM provides a more accurate simulation of SEDDS digestion (at least in terms of droplet size) than conventional USP 2 apparatus (Mercuri et al., 2008). A second study assessed the relative performance of gelatin and HPMC capsules in the fed and fasted states. It was concluded that the capture rupture times obtained from the DGM were similar to those observed by in vivo gamma scintigraphy in the fasted state and were delayed in the fed state, although the comparison to in vivo scintigraphy results in this case was affected by the impact of food on the dispersion of contents and subsequent sampling in the DGM (Vardakou et al., 2011b). The DGM has also been used to assess the release of a complex dosage form containing several drugs in immediate-release and controlled-release layers with some advantages observed for prediction of performance over conventional USP 2 dissolution apparatus (Mann and Pygall, 2012). Clearly more studies are required to reach a judgment on the value of this system but its ability to simulate gastric forces and meal processing should have value in accurately comparing the relative performance of clinical formulations and in particular quantifying the potential for food-effects and gastric retention of gastro-retentive dosage forms. 


\subsubsection{TNO gastro-Intestinal Model (TIM)}

The TIM-1 system (Figure 8) is a multi-compartmental, dynamic, computer-controlled model of the human upper gastrointestinal tract (Minekus et al., 1995). The TIM-1 system simulates the physiological conditions of the stomach and small intestine, including the dynamics of mixing, gastric emptying and intestinal transit times, the gastric and intestinal $\mathrm{pH}$ values, body temperature, and the composition and activity of the secretion fluids. In addition, low molecular weight compounds are removed continuously from the jejunal and ileal compartments of the system via dialysis or filtration membrane systems. This allows quantification of the bioaccessibility (i.e. the amount of digested product or drug substance in solution and therefore available for absorption) (Blanquet et al., 2004; Souliman et al., 2006, 2007). The use of these membrane systems means that in vivo processes such as active transport, efflux and intestinal wall metabolism are not modeled mechanistically by the system. For this purpose, TIM-1 samples can be combined with intestinal absorption systems to predict oral bioavailability (Déat et al., 2009; Haraldsson et al., 2005; Verwei et al., 2006).

Hydrodynamics are controlled by changes in water pressure on flexible membranes which contain the luminal contents and enable mixing by alternate cycles of compression and relaxation, simulating in vivo muscular peristaltic contractions. Additionally, transit is regulated by opening or closing peristaltic valves that connect each compartment, allowing the controlled passage of liquids and food/drug particle.

While there are many examples where TIM-1 has been used to study the digestion and bioaccessibility of nutritional compounds over the last few years (e.g. Chen et al., 2010; Krul et al., 2000; Lila et al., 2011; Verwei et al., 2003, 2006), there are only a limited number of examples in the literature describing its use for the evaluation of pharmaceutical dosage forms. Blanquet et al. and Souliman et al. used TIM-1 to evaluate the impact of transit time and food on the absorption of paracetamol and theophylline following administration as either the free powder form or as a sustained release tablets (Blanquet et al., 2004; Souliman et al., 2006, 2007). These studies demonstrated that the profiles of jejunal absorption found in vitro were consistent with in vivo data and a good correlation was seen with Tmax values for the immediate-release form. It was also shown that food intake (in the form of a standard breakfast) reduced the amount of paracetamol available for absorption. This was judged to be similar to clinical studies which showed a lower Cmax and delayed Tmax in the fed state compared to intake with water in the fasted state (Ameer et al., 1983; Divoll et al., 1982; 
Rostami-Hodjegan et al., 2002a; Rygnestad et al., 2000). A further study evaluated the use of this dynamic model to improve the predictability of physiologically-based pharmacokinetic (PBPK) simulation and modeling software for a paroxetine hydrochloride immediate-release tablet (Naylor et al., 2006). Using the bioaccessibility profile from TIM-1 instead of classic USP 2 dissolution data as input rate for the in silico absorption model, improved the predicted plasma profile. Whereas the TIM-1 system provides information on the bioaccessibility of a compound during passage through the upper GI tract, a combination with TNO's TIM-2 system, simulating the physiological conditions in the large intestine, enables an investigation of the release of a compound through the entire GI tract. As an example, a study with 5aminosalicylate (5-ASA, mesalamine) was performed to investigate its release kinetics from a tablet with pH-dependent, gastroresistant coating under fasted and fed state conditions (Tenjarla et al., 2007). The results demonstrated that 5-ASA release under simulated small intestinal conditions was minimal, while its release was high after entering the TIM-2 system, which corresponds with clinical data.

In TIM-1, lipid digestion and the bioaccessibility of lipid-soluble compounds is studied by applying filtration through a $50 \mathrm{~nm}$ pore filter at a predetermined filtration rate. The use of this filter allows removal of mixed micelles containing lipophilic compounds such as products of fat digestion and drugs, while undigested fat and undissolved compounds are retained in the luminal compartment (Minekus et al., 2005; Reis et al., 2008). This set-up was also recently applied in a study with an immediate-release fosamprenavir tablet (Brouwers et al., 2011) tested under fed state and fasted state conditions in TIM-1. The study shows that disintegration and fosamprenavir dissolution was significantly postponed in the fed state compared to the fasted state. This resulted in a lag in the appearance of bioaccessible fosamprenavir but no effect on the cumulative bioaccessibility. These results were in agreement with the data observed in a study with healthy volunteers. Furthermore, a study by Dickinson et al. demonstrated that it is possible to study formulations delivering poorly soluble compounds using TIM-1. It was shown that it was possible to predict the performance of a BCS class II compound in both fasting and achlorhydric conditions (Dickinson et al., 2012). These examples suggest that the GI-modeling system, which provides an advanced level of control over a dynamic and complex luminal environment, may have several advantages over conventional dissolution methodologies when assessing the performance of oral formulations in either the fasted or fed state. 
Most scientists have reported good correlation between product performance in the TIM-1 system and clinical performance. Dickinson and co-workers noted that the TIM-1 stomach was developed for rapidly and well dispersing food products (Dickinson et al., 2012). If the pharmaceutical product under development does not meet this criterion, the data generated may not be representative of clinical performance and should be interpreted cautiously. The advanced gastric compartment, a new development from TNO intended to address this concern, is specially designed to study the behaviour of food and dosage forms including a realistic gastric shape and mechanical forces. The compartment consists of a body part with a flexible wall that gradually contracts to simulate gastric tone and consequent reduction of gastric volume during emptying. A flexible bottom can be moved in conjunction with contractions of an antral unit to simulate antral mixing. A valve is synchronized with antral mixing to simulate the opening of the pyloric sphincter during gastric emptying.

Besides accurate simulation of the gastric shear and pressure forces, relevant for drug release of particular formulations, two other divergences of the TIM-1 system from the clinical situation are:

- No drug removal from the duodenal compartment by filtration or dialysis;

- The total volume of fluid in all small intestinal compartments of the TIM-1 together is $\sim 300 \mathrm{~mL}$. Although this volume of fluid in the small intestine is realistic, it does not represent the in vivo distribution of fluid in small pockets (Schiller et al., 2005); the high liquid volumes in TIM-1 system small intestinal compartments may mean a theoretical risk that products that have sub-optimal disintegration or wetting properties exhibit better performance in the TIM-1 than in the clinic.

Finally, the throughput limitations of the TIM-1 system are being addressed in the design of a new apparatus known as the tiny-TIM system. In this apparatus, the gastric and small intestinal conditions (duodenum, jejunum and ileum) are simulated through the use of two compartments instead of four (TIM-1).

\section{In vitro models for predicting drug absorption: the future}


As oral drugs continue to be the largest segment of new drug approvals, with more than half of the newly approved medicines in the USA 2012 being delivered orally ("New Drug Approvals," 2013), novel, biorelevant in vitro tools for the prediction of oral dosage form performance in vivo have a key role in making drug product development more efficient. Novel biopharmaceutical tools could lead to:

i) Preventing potential development compounds from being falsely discarded in the preclinical development phase through lack of appropriate identification of problems with drug release from the dosage form in the GI tract

ii) Enabling development options to be offered early in the preclinical phase to support an oral dosage form including the need for enhanced formulations

iii) More realistic assessment of licensed-in compounds

iv) Assessing the need/potential for dosage forms with modified release

v) Reducing the need for animal or human bridging studies between clinical trial formulations and reduce risk of failure in late stage pivotal BE studies

vi) QC tests that are more clinically relevant and thereby offer opportunities for more cost-effective manufacturing.

As can be inferred from the foregoing sections, there is an on-going dilemma facing the pharmaceutical industry in terms of which in vitro tests are needed in order to predict the behaviour of dosage forms in the GI tract. An ideal test would be one in which:

1) all aspects of in vivo complexity that might affect drug release and absorption of drug from the GI tract are simulated in one test, and

2) the test is so simple to use that it not only can be invoked in development but also be used as a QC test for the manufactured product.

However, it is not possible to combine both of these objectives (complexity versus simplicity) in one single test. Instead, test methods used to assess oral products in development need to be tailored to the product and the risks specific to it. In addition, just as the product evolves during development where further information characterizing its behavior is obtained, the in vitro tests being applied to the product should also evolve correspondingly.

What this means in practice is that the tools needed for in vitro assessment in the future are likely to be diverse, sometimes focusing on a single aspect of the in vivo environment likely to be critical to that product, and on other occasions needing to be capable of mimicking 
multiple aspects of importance to in vivo performance. In other words a "toolkit" of in vitro tests is needed, with tools of varying complexity, along with a better understanding of when to use which tool for which product, and at which stage of development.

With respect to dosage forms that should release the drug as quickly as possible, this review has revealed a number of significant gaps that must be addressed to establish a "tool-kit" of release tests that can be accessed according to the characteristics of the drug and mechanism of release from the dosage form. Given the preponderance of poorly soluble compounds in today's pharmaceutical pipelines, the questions that have highest priority include how best to:

1) evaluate the extent to which the dissolution rate of the drug from the dosage form is limiting to the absorption;

2) assess whether a poorly soluble drug will precipitate either in the stomach or upon entering the small intestine, and if so, how to predict this, and test which is the best formulation strategy to circumvent this;

3) assess the interplay among drug characteristics (solubility, particle size, dissolution, permeability) and physiological considerations (gastric emptying, hydrodynamics, and permeability) in determining the extent of absorption; and

4) assess whether the chosen dosage formulation will exhibit a food effect.

To answer the first question, we must first delve deeper into defining the issues, such as whether sink conditions are appropriate, what volumes of fluids should be used, what complexity of composition is appropriate and how hydrodynamic observations in vivo can be translated into the design of release tests. For certain, the traditional, compendial tests do not address these issues adequately - the simple setups and buffer systems are far from appropriate to reflect the complex conditions in vivo under which the dosage form must release a poorly soluble drug.

For the second question, the interaction between the excipients and the drug is paramount to the mechanism of release and thus to the design of the release test. For enabling formulations, tailor-made release tests need to be designed, whether this be taking into account digestion effects for lipid-based dosage forms or applying appropriate sample handling techniques in the case of formulations housing nanosized drug. Again here, the traditional test conditions used for QC purposes aim for sink conditions and therefore are not set up to study behaviour at or above saturation, which may be fundamental to understanding the in vivo performance of enabling dosage forms (Miller et al., 2011). 
As already discussed, there are several approaches to evaluating the interplay of drug, excipients and physiology and its effect on the overall uptake of the drug, ranging from models that attempt to couple release with absorption (either directly or indirectly) to integration of data from various sources using PBPK models. Particularly when addressing this question it is important to consider the relative merits of in vitro sophistication vis a vis in silico sophistication.

Looking at the physiological considerations we see the real Achilles heel in the application of in vitro release tests to predicting in vivo performance - the hydrodynamics issue. Although the human gut has been studied to some degree in terms of flow rates and pressures generated, and modeled several ways both in silico and in vitro with respect to hydrodynamics, our basis for choosing a particular apparatus and hydrodynamic set-up is still largely empirical.

Then we have the food effect question. As in other aspects of oral drug release and absorption, animal models are notoriously fickle or difficult to invoke with respect to food effects and so we are left with trying to mimic the upper human GI tract with in vitro models that are often unsatisfactory in terms of their ability to predict this important effect. Too simple and we get no read-out at all (compendial methods), too complex and the experiments become very time and cost intensive. And here too, with many models, correlations in vivo to in vitro are anecdotal rather than being mechanistically derived and generally applicable.

Following on to formulations with MR, it is logical that since the dosage form is intended to release at a specific position in the GI tract or over a larger section of the GI tract, creating models which can predict in vivo performance is even more complex. As pointed out in section 3.3.1, the GI tract has been less well characterized in most aspects as one proceeds further along. So although we are reasonably well informed about volumes, flow rates, composition etc. in the stomach and upper small intestine, the analogous description of conditions in the ileum and colon is sketchy at best. In particular, the impact of motility on the performance of dosage forms with MR is an area where an improvement in knowledge base and experimental design is sorely needed. Furthermore, the factors governing the absorption of drugs in the lower regions of the gut are still not well understood. Some methods are emerging [e.g. for microbiotic stability ("ProDigest - Gastrointestinal Expertise," 2013), e.g. for permeability via a modified Caco-2 assay (Tannergren et al., 2009)], but it will take some time to refine and validate these. The general lack of information about the lower gut makes it extremely difficult to develop an accurate, one-size-fits-all model of the entire GI tract in the 
laboratory and assumptions invariably have to be made. No doubt within the next decade or so we will learn quite a bit more about these regions and how dosage forms and drugs interact with them and as a result be able to design better models, but these models will be almost certainly even more complex than the most sophisticated models that exist today.

In the meantime, perhaps the best way forward is to consider the mechanism of release of the dosage form, identify the associated key parameters which can influence the pattern and rate of release and work towards models that can address primarily those parameters. For example, if the mechanism of release is not sensitive to hydrodynamics, which seems to be the case for at least the elementary osmotic pump formulations, there is little sense in trying to develop a highly refined hydrodynamic model to study the release. And as a second example, if the release is governed by the erosion of a polymer which acts as a matrix depot for the drug, it would be prudent to examine the sensitivities of the polymer erosion rate, to the changing composition of the gastrointestinal fluids as the dosage form moves through the gut. As a result, it is reasonable to expect to develop a decision tree for dosage forms with MR patterns, in which the test conditions would be matched to the sensitivities of the release mechanism.

For both IR and MR dosage forms, there is yet another issue that has faced scientists in applying biorelevant tests to the development of oral products. To improve the efficiency of drug development, it may be preferable to use more complex tests at relatively early stages of formulation development. Then, after the optimal formulation has been developed, a simpler test can be adopted that focuses on the sensitivities of the final formulation later in development. This logic can run counter to the general thinking frequently being applied in drug product development today where the tests are kept simple in the early stages in order to reduce development costs. However, the advantages of reliably predicting in vivo performance early not only allows for rational formulation selection but also to identify which attributes are most critical to ensure consistent in vivo performance. As a result, determining which in vitro tool is not only going to adequately predict in vivo performance, but also is going to be most cost effective and efficient, is important.

\section{$5 \quad$ Integration of in vitro models with PBPK modeling}

Although results of biorelevant dissolution release tests have proven useful for qualitatively, and in some cases quantitatively predicting in vivo drug performance (Fang et al., 2010; Sunesen et al., 2005), the dissolution release test cannot capture all processes that may affect the in vivo performance. For example, gastric emptying, permeability through the intestinal 
membrane, transit time, $\mathrm{pH}$ and fluid volume in each segment of the GI tract, first pass metabolism and excretion can all play a role in drug bioavailability in addition to the release from the dosage form. To put all these influences in better perspective, the in vitro test results can be combined with physiologically based pharmacokinetic (PBPK) models. For example, Shono et al. used this approach to explain why the food effect of celecoxib, a lipophilic BCS class 2 drug, was much more modest than is typical of this class (Shono et al., 2009). Similarly, Wei and Löbenberg explored biorelevant dissolution coupled with PBPK to understand the variable PK behaviour of an antidiabetic drug (Wei and Löbenberg, 2006). This approach has even found some resonance in the regulatory area, with Okumu et al. proposing a combination of dissolution tests with PBPK as a surrogate for in vivo drug assessment (Okumu et al., 2009). When biorelevant in vitro testing is combined with PBPK modelling, it opens up the possibility of estimating the complete in vivo drug plasma profile rather than just the rate and/or extent of absorption (Nicolaides et al., 2001; Okumu et al., 2009; Shono et al., 2009; Takano et al., 2010; Wei and Löbenberg, 2006). Such an approach could be a very interesting tool for product development within the QbD paradigm since it offers a way of linking in vitro and clinical performance..

\section{Concluding remarks}

Methods for characterizing drug release from oral pharmaceutical products have evolved substantially since they were first introduced as quality control tools. Although the classical quality control methods can detect variations in manufacturing procedures, they may or may not be relevant to the in vivo performance of the drug product. As we have gained deeper insight into gastrointestinal physiology from the pharmaceutical perspective over the last 2025 years, it has become possible to develop more biorelevant methods, which can better reflect in vivo performance of the dosage form. This has in turn enabled us to align in vitro methods with the more complex and innovative dosage forms being developed to meet the needs of modern drug substances.

A second force in triggering the development of more sophisticated methods to test for drug product performance is the clear trend towards the Quality by Design (QbD) paradigm. According to this paradigm, the dosage form should be developed with in vivo performance in mind using knowledge about the drug profile required at the site of action on the one hand and appropriate in vitro tools to ensure that the dosage form developed can deliver this profile on 
the other hand. A leading tenet of $\mathrm{QbD}$ is a shift away from testing batch quality "after the fact" and towards building quality into the dosage form and using in-process controls to ensure that the batch manufacture falls within the pre-defined design space and thus can be released to the market. As a consequence, many components of the classical, quality control oriented in vitro release methodologies may become less relevant for the development of pharmaceutical formulations in the future. However, the QbD paradigm is contingent on the availability of a set of in vivo relevant tests to build understanding around the influence of critical formulation factors on clinical performance during development.

The vision of addressing current gaps in, as well as anticipating future needs for, in vitro testing of formulations is part and parcel of the proposed IMI OrBiTo project. The state of the art, as described in this review, identifies the in vitro methods which are currently used to address the performance of oral drug products in the classical QC context and in the context of product development through the application of biorelevant conditions. This latter group of tests is important not only as they apply to formulation characterization and design but also as they provide input to PBPK and other in silico based models to facilitate the prediction of the in vivo performance of drug products. The combined in vitro/PBPK approach can be expected to allow us to take the next step beyond BCS to predict influence of dissolution performance on in vivo PK. This area must expand not only with respect to improving the understanding of GI physiology and drug absorption but also with respect to appropriately describing the release from "enabled" dosage forms such as those based on nanotechnology, amorphous solid dispersions, lipid-based and modified release systems, to name just a few.

Horizon scanning exercises suggest that while tremendous progress has been made, new or optimized in vitro tools are still needed. As addressed in the gap analysis, the resulting "toolbox" should include methods which can accurately simulate biorelevant processes as GI digestion, motility and absorption as well as being able to deal with supersaturation, excipient digestion and nanodispersions. Additionally, to streamline and optimize oral drug product development, we will need to develop an understanding of when and where these tools are best applied - preferably in the form of a decision tree which can be implemented according to both the clinical needs and the drug substance properties. While some of the more sophisticated tools that are being developed may become "niche" tools to be used in very specific circumstances, simpler screening tools might be implemented on a much broader basis in oral drug development. For example, for many formulations of weak bases, a simple method for screening resistance to precipitation upon entry into the small intestine may be 
sufficient, but in cases where the drug proves to be very prone to precipitation, a full-blown testing method mimicking not only a physiological transfer rate into the small intestine but also the balance between transfer and absorption may become necessary. Likewise, different levels of test sophistication will be appropriate, depending on whether one is taking the drug through the first formulation screens or, at the other end of the scale, one is trying to optimize performance in a specific clinical subpopulation.

The combination of such a "toolbox" of methods and a decision tree for their implementation based on the indication and drug properties is expected to significantly improve and accelerate the translation of important new drugs to the patient.

\section{Acknowledgements}

This work has received support from the Innovative Medicines Initiative Joint Undertaking (http://www.imi.europa.eu) under grant agreement $\mathrm{n}^{\circ} 115369$, resources of which are composed of financial contribution from the European Union's Seventh Framework Programme (FP7/2007-2013) and EFPIA companies' in kind contribution.

\section{References}

Abrahamsson, B., Albery, T., Eriksson, A., Gustafsson, I., Sjöberg, M., 2004. Food effects on tablet disintegration. Eur J Pharm Sci 22, 165-172.

Abrahamsson, B., Alpsten, M., Bake, B., Larsson, A., Sjögren, J., 1998. In vitro and in vivo erosion of two different hydrophilic gel matrix tablets. Eur J Pharm Biopharm 46, 69-75.

Abrahamsson, B., Pal, A., Sjöberg, M., Carlsson, M., Laurell, E., Brasseur, J.G., 2005. A novel in vitro and numerical analysis of shear-induced drug release from extended-release tablets in the fed stomach. Pharm. Res. 22, 1215-1226.

Ameer, B., Divoll, M., Abernethy, D.R., Greenblatt, D.J., Shargel, L., 1983. Absolute and relative bioavailability of oral acetaminophen preparations. J Pharm Sci 72, 955-958.

Amidon, G.L., Lennernäs, H., Shah, V.P., Crison, J.R., 1995. A theoretical basis for a biopharmaceutic drug classification: the correlation of in vitro drug product dissolution and in vivo bioavailability. Pharm. Res. 12, 413-420.

Anby, M.U., Williams, H.D., McIntosh, M.P., Benameur, H., Edwards, G.A., Pouton, C.W., Porter, C.J., 2012. Lipid digestion as a trigger for supersaturation: evaluation of the impact of 
supersaturation stabilization on the in vitro and in vivo performance of self-emulsifying drug delivery systems. Mol. Pharm. 9, 2063-2079.

Aoki, S., Ando, H., Tatsuishi, K., Uesugi, K., Ozawa, H., 1993. Determination of the mechanical impact force in the in vitro dissolution test and evaluation of the correlation between in vivo and in vitro release. International Journal of Pharmaceutics 95, 67-75.

Aoki, S., Uesugi, K., Tatsuishi, K., Ozawa, H., Kayano, M., 1992. Evaluation of the correlation between in vivo and in vitro release of phenylpropanolamine $\mathrm{HCl}$ from controlledrelease tablets. International Journal of Pharmaceutics 85, 65-73.

Armand, M., Borel, P., Dubois, C., Senft, M., Peyrot, J., Salducci, J., Lafont, H., Lairon, D., 1994. Characterization of emulsions and lipolysis of dietary lipids in the human stomach. Am. J. Physiol. 266, G372-381.

Arnold, Y.E., Imanidis, G., Kuentz, M.T., 2011. Advancing in-vitro drug precipitation testing: new process monitoring tools and a kinetic nucleation and growth model. J. Pharm. Pharmacol. 63, 333-341.

Arrowsmith, J., 2011a. Trial watch: phase III and submission failures: 2007-2010. Nat Rev Drug Discov 10, 87.

Arrowsmith, J., 2011b. Trial watch: Phase II failures: 2008-2010. Nat Rev Drug Discov 10, 328-329.

Astashkina, A., Mann, B., Grainger, D.W., 2012. A critical evaluation of in vitro cell culture models for high-throughput drug screening and toxicity. Pharmacol. Ther. 134, 82-106.

Bai, G., Armenante, P.M., Plank, R.V., Gentzler, M., Ford, K., Harmon, P., 2007. Hydrodynamic investigation of USP dissolution test apparatus II. J Pharm Sci 96, 2327-2349.

Baxter, J.L., Kukura, J., Muzzio, F.J., 2005a. Hydrodynamics-induced variability in the USP apparatus II dissolution test. Int J Pharm 292, 17-28.

Baxter, J.L., Kukura, J., Muzzio, F.J., 2005b. Shear-induced variability in the United States Pharmacopeia Apparatus 2: modifications to the existing system. AAPS J 7, E857-864.

Beig, A., Miller, J.M., Dahan, A., 2012. Accounting for the solubility-permeability interplay in oral formulation development for poor water solubility drugs: the effect of PEG-400 on carbamazepine absorption. Eur J Pharm Biopharm 81, 386-391.

Bevernage, J., Brouwers, J., Annaert, P., Augustijns, P., 2012a. Drug precipitation-permeation interplay: supersaturation in an absorptive environment. Eur J Pharm Biopharm 82, 424-428.

Bevernage, J., Brouwers, J., Brewster, M.E., Augustijns, P., 2012b. Evaluation of gastrointestinal drug supersaturation and precipitation: Strategies and issues. Int J Pharm.

Bhattachar, S.N., Perkins, E.J., Tan, J.S., Burns, L.J., 2011. Effect of gastric pH on the pharmacokinetics of a BCS class II compound in dogs: utilization of an artificial stomach and duodenum dissolution model and GastroPlus, ${ }^{\mathrm{TM}}$ simulations to predict absorption. $\mathrm{J}$ Pharm Sci 100, 4756-4765. 
Blanquet, S., Zeijdner, E., Beyssac, E., Meunier, J.-P., Denis, S., Havenaar, R., Alric, M., 2004. A dynamic artificial gastrointestinal system for studying the behavior of orally administered drug dosage forms under various physiological conditions. Pharm. Res. 21, 585591.

Brouwers, J., Anneveld, B., Goudappel, G.-J., Duchateau, G., Annaert, P., Augustijns, P., Zeijdner, E., 2011. Food-dependent disintegration of immediate release fosamprenavir tablets: in vitro evaluation using magnetic resonance imaging and a dynamic gastrointestinal system. Eur J Pharm Biopharm 77, 313-319.

Brouwers, J., Brewster, M.E., Augustijns, P., 2009. Supersaturating drug delivery systems: the answer to solubility-limited oral bioavailability? J Pharm Sci 98, 2549-2572.

Brouwers, J., Tack, J., Lammert, F., Augustijns, P., 2006. Intraluminal drug and formulation behavior and integration in in vitro permeability estimation: a case study with amprenavir. $\mathrm{J}$ Pharm Sci 95, 372-383.

Brown, C.K., Friedel, H.D., Barker, A.R., Buhse, L.F., Keitel, S., Cecil, T.L., Kraemer, J., Morris, J.M., Reppas, C., Stickelmeyer, M.P., Yomota, C., Shah, V.P., 2011. FIP/AAPS joint workshop report: dissolution/in vitro release testing of novel/special dosage forms. AAPS PharmSciTech 12, 782-794.

Buch, P., Langguth, P., Kataoka, M., Yamashita, S., 2009. IVIVC in oral absorption for fenofibrate immediate release tablets using a dissolution/permeation system. J Pharm Sci 98, 2001-2009.

Carino, S.R., Sperry, D.C., Hawley, M., 2006. Relative bioavailability estimation of carbamazepine crystal forms using an artificial stomach-duodenum model. J Pharm Sci 95, $116-125$.

Carino, S.R., Sperry, D.C., Hawley, M., 2010. Relative bioavailability of three different solid forms of PNU-141659 as determined with the artificial stomach-duodenum model. J Pharm Sci 99, 3923-3930.

Carlert, S., Pålsson, A., Hanisch, G., von Corswant, C., Nilsson, C., Lindfors, L., Lennernäs, H., Abrahamsson, B., 2010. Predicting intestinal precipitation--a case example for a basic BCS class II drug. Pharm. Res. 27, 2119-2130.

Carriere, F., Barrowman, J.A., Verger, R., Laugier, R., 1993. Secretion and contribution to lipolysis of gastric and pancreatic lipases during a test meal in humans. Gastroenterology 105, 876-888.

Castela-Papin, N., Cai, S., Vatier, J., Keller, F., Souleau, C.H., Farinotti, R., 1999. Drug interactions with diosmectite: a study using the artificial stomach-duodenum model. Int $\mathbf{J}$ Pharm 182, 111-119.

Center for Drug Evaluation and Research, 2008. Guidance for Industry Orally Disintegrating Tablets. 
Center for Drug Evaluation and Research, 2013. Drug Innovation - New Molecular Entity $\begin{array}{lllll}\text { Approvals } & \text { for } & 2012 & \text { [WWW } & \text { Document] }\end{array}$ http://www.fda.gov/Drugs/DevelopmentApprovalProcess/DrugInnovation/ucm336115.htm

Chen, L., Hébrard, G., Beyssac, E., Denis, S., Subirade, M., 2010. In vitro study of the release properties of soy-zein protein microspheres with a dynamic artificial digestive system. J. Agric. Food Chem. 58, 9861-9867.

Christiansen, A., Backensfeld, T., Weitschies, W., 2010. Effects of non-ionic surfactants on in vitro triglyceride digestion and their susceptibility to digestion by pancreatic enzymes. Eur $\mathbf{J}$ Pharm Sci 41, 376-382.

Clarysse, S., Tack, J., Lammert, F., Duchateau, G., Reppas, C., Augustijns, P., 2009. Postprandial evolution in composition and characteristics of human duodenal fluids in different nutritional states. J Pharm Sci 98, 1177-1192.

Cole, E.T., Scott, R.A., Cade, D., Connor, A.L., Wilding, I.R., 2004. In vitro and in vivo pharmacoscintigraphic evaluation of ibuprofen hypromellose and gelatin capsules. Pharm. Res. 21, 793-798.

Cuiné, J.F., Charman, W.N., Pouton, C.W., Edwards, G.A., Porter, C.J.H., 2007. Increasing the proportional content of surfactant (Cremophor EL) relative to lipid in self-emulsifying lipid-based formulations of danazol reduces oral bioavailability in beagle dogs. Pharm. Res. $24,748-757$.

Cuiné, J.F., McEvoy, C.L., Charman, W.N., Pouton, C.W., Edwards, G.A., Benameur, H., Porter, C.J.H., 2008. Evaluation of the impact of surfactant digestion on the bioavailability of danazol after oral administration of lipidic self-emulsifying formulations to dogs. J Pharm Sci 97, 995-1012.

Curatolo, W., Nightingale, J.A., Herbig, S.M., 2009. Utility of hydroxypropylmethylcellulose acetate succinate (HPMCAS) for initiation and maintenance of drug supersaturation in the GI milieu. Pharm. Res. 26, 1419-1431.

D’Arcy, D.M., Corrigan, O.I., Healy, A.M., 2005. Hydrodynamic simulation (computational fluid dynamics) of asymmetrically positioned tablets in the paddle dissolution apparatus: impact on dissolution rate and variability. J. Pharm. Pharmacol. 57, 1243-1250.

Dahan, A., Hoffman, A., 2007. The effect of different lipid based formulations on the oral absorption of lipophilic drugs: the ability of in vitro lipolysis and consecutive ex vivo intestinal permeability data to predict in vivo bioavailability in rats. Eur J Pharm Biopharm 67, 96-105.

Déat, E., Blanquet-Diot, S., Jarrige, J.-F., Denis, S., Beyssac, E., Alric, M., 2009. Combining the dynamic TNO-gastrointestinal tract system with a Caco-2 cell culture model: application to the assessment of lycopene and alpha-tocopherol bioavailability from a whole food. $\mathrm{J}$. Agric. Food Chem. 57, 11314-11320.

Diakidou, A., Vertzoni, M., Goumas, K., Söderlind, E., Abrahamsson, B., Dressman, J., Reppas, C., 2009. Characterization of the contents of ascending colon to which drugs are exposed after oral administration to healthy adults. Pharm. Res. 26, 2141-2151. 
Dickinson, P.A., Abu Rmaileh, R., Ashworth, L., Barker, R.A., Burke, W.M., Patterson, C.M., Stainforth, N., Yasin, M., 2012. An investigation into the utility of a multicompartmental, dynamic, system of the upper gastrointestinal tract to support formulation development and establish bioequivalence of poorly soluble drugs. AAPS J 14, 196-205.

Diebold, S., Dressman, J., 2001. Hydrodynamik kompendialer LösungsgeschwindigkeitsTestapparaturen. Pharm Ind 63, 94-104.

Digenis, G.A., Sandefer, E.P., Page, R.C., Doll, W.J., Gold, T.B., Darwazeh, N.B., 2000. Bioequivalence study of stressed and nonstressed hard gelatin capsules using amoxicillin as a drug marker and gamma scintigraphy to confirm time and GI location of in vivo capsule rupture. Pharm. Res. 17, 572-582.

Divoll, M., Greenblatt, D.J., Ameer, B., Abernethy, D.R., 1982. Effect of food on acetaminophen absorption in young and elderly subjects. J Clin Pharmacol 22, 571-576.

Dressman, J.B., Amidon, G.L., Reppas, C., Shah, V.P., 1998. Dissolution testing as a prognostic tool for oral drug absorption: immediate release dosage forms. Pharm. Res. 15, 1122.

Dressman, J.B., Krämer, J., 2005. Pharmaceutical Dissolution Testing. Taylor \& Francis.

Eaton, J.W., Tran, D., Hauck, W.W., Stippler, E.S., 2012. Development of a performance verification test for USP apparatus 4. Pharm. Res. 29, 345-351.

Erceg, M., Vertzoni, M., Cerić, H., Dumić, M., Cetina-Čižmek, B., Reppas, C., 2012. In vitro vs. canine data for assessing early exposure of doxazosin base and its mesylate salt. Eur $\mathbf{J}$ Pharm Biopharm 80, 402-409.

European Medical Agency, 2010. committee for medicinal products for human use: Guideline on the Investigation of Bioequivalence Accessed on December 5, 2012.

Fang, J.B., Robertson, V.K., Rawat, A., Flick, T., Tang, Z.J., Cauchon, N.S., McElvain, J.S., 2010. Development and application of a biorelevant dissolution method using USP apparatus 4 in early phase formulation development. Mol. Pharm. 7, 1466-1477.

Fatouros, D.G., Nielsen, F.S., Douroumis, D., Hadjileontiadis, L.J., Mullertz, A., 2008. In vitro-in vivo correlations of self-emulsifying drug delivery systems combining the dynamic lipolysis model and neuro-fuzzy networks. Eur J Pharm Biopharm 69, 887-898.

Fernandez, S., Chevrier, S., Ritter, N., Mahler, B., Demarne, F., Carrière, F., Jannin, V., 2009. In vitro gastrointestinal lipolysis of four formulations of piroxicam and cinnarizine with the self emulsifying excipients Labrasol and Gelucire 44/14. Pharm. Res. 26, 1901-1910.

Fernandez, S., Jannin, V., Rodier, J.-D., Ritter, N., Mahler, B., Carrière, F., 2007.

Comparative study on digestive lipase activities on the self emulsifying excipient Labrasol, medium chain glycerides and PEG esters. Biochim. Biophys. Acta 1771, 633-640.

Fleisher, D., Li, C., Zhou, Y., Pao, L.H., Karim, A., 1999. Drug, meal and formulation interactions influencing drug absorption after oral administration. Clinical implications. Clin Pharmacokinet 36, 233-254. 
Food and Drug Administration, 2009. FDA Guidance: Waiver of in vivo bioavailability and bioequivalence studies for immediate-release solid oral dosage forms based on biopharmaceutics classification system.

Fotaki, N., Aivaliotis, A., Butler, J., Dressman, J., Fischbach, M., Hempenstall, J., Klein, S., Reppas, C., 2009. A comparative study of different release apparatus in generating in vitro-in vivo correlations for extended release formulations. Eur J Pharm Biopharm 73, 115-120.

Fotaki, N., Symillides, M., Reppas, C., 2005a. In vitro versus canine data for predicting input profiles of isosorbide-5-mononitrate from oral extended release products on a confidence interval basis. Eur J Pharm Sci 24, 115-122.

Fotaki, N., Symillides, M., Reppas, C., 2005b. Canine versus in vitro data for predicting input profiles of L-sulpiride after oral administration. Eur J Pharm Sci 26, 324-333.

Fotaki, N., Vertzoni, M., 2010. Biorelevant dissolution methods and their applications in in vitro- in vivo correlations for oral formulations - Opus. The Open Drug Delivery Journal 213.

Frank, K.J., Rosenblatt, K.M., Westedt, U., Hölig, P., Rosenberg, J., Mägerlein, M., Fricker, G., Brandl, M., 2012. Amorphous solid dispersion enhances permeation of poorly soluble ABT-102: true supersaturation vs. apparent solubility enhancement. Int J Pharm 437, 288293.

Galia, E., Horton, J., Dressman, J.B., 1999. Albendazole generics--a comparative in vitro study. Pharm. Res. 16, 1871-1875.

Galia, E., Nicolaides, E., Hörter, D., Löbenberg, R., Reppas, C., Dressman, J.B., 1998. Evaluation of various dissolution media for predicting in vivo performance of class I and II drugs. Pharm. Res. 15, 698-705.

Garbacz, G., Golke, B., Wedemeyer, R.-S., Axell, M., Söderlind, E., Abrahamsson, B., Weitschies, W., 2009. Comparison of dissolution profiles obtained from nifedipine extended release once a day products using different dissolution test apparatuses. Eur J Pharm Sci 38, $147-155$.

Garbacz, G., Klein, S., Weitschies, W., 2010. A biorelevant dissolution stress test device background and experiences. Expert Opin Drug Deliv 7, 1251-1261.

Garbacz, G., Wedemeyer, R.-S., Nagel, S., Giessmann, T., Mönnikes, H., Wilson, C.G., Siegmund, W., Weitschies, W., 2008. Irregular absorption profiles observed from diclofenac extended release tablets can be predicted using a dissolution test apparatus that mimics in vivo physical stresses. Eur J Pharm Biopharm 70, 421-428.

Gargouri, Y., Pieroni, G., Riviere, C., Sauniere, J.F., Lowe, P.A., Sarda, L., Verger, R., 1986. Kinetic assay of human gastric lipase on short- and long-chain triacylglycerol emulsions. Gastroenterology 91, 919-925. 
Ginski, M.J., Polli, J.E., 1999. Prediction of dissolution-absorption relationships from a dissolution/Caco-2 system. Int J Pharm 177, 117-125.

GMP Guideline Quality of Modified Release Products A) Oral Solid Dosage Forms B) Transdermal Dosage Forms Section I (Quality) CPMP/QWP/ 604/96 [WWW Document], 2013. URL http://www.gmp-compliance.org/eca_guideline_3502.html

Goodman, K., Hodges, L.A., Band, J., Stevens, H.N.E., Weitschies, W., Wilson, C.G., 2010. Assessing gastrointestinal motility and disintegration profiles of magnetic tablets by a novel magnetic imaging device and gamma scintigraphy. Eur J Pharm Biopharm 74, 84-92.

Gray, V., Kelly, G., Xia, M., Butler, C., Thomas, S., Mayock, S., 2009. The science of USP 1 and 2 dissolution: present challenges and future relevance. Pharm. Res. 26, 1289-1302.

Gribbon, P., Sewing, A., 2005. High-throughput drug discovery: what can we expect from HTS? Drug Discov. Today 10, 17-22.

Grundy, J.S., Foster, R.T., 1996. The nifedipine gastrointestinal therapeutic system (GITS). Evaluation of pharmaceutical, pharmacokinetic and pharmacological properties. Clin Pharmacokinet 30, 28-51.

Gu, C.-H., Rao, D., Gandhi, R.B., Hilden, J., Raghavan, K., 2005. Using a novel multicompartment dissolution system to predict the effect of gastric $\mathrm{pH}$ on the oral absorption of weak bases with poor intrinsic solubility. J Pharm Sci 94, 199-208.

Guzmán, H.R., Tawa, M., Zhang, Z., Ratanabanangkoon, P., Shaw, P., Gardner, C.R., Chen, H., Moreau, J.-P., Almarsson, O., Remenar, J.F., 2007. Combined use of crystalline salt forms and precipitation inhibitors to improve oral absorption of celecoxib from solid oral formulations. J Pharm Sci 96, 2686-2702.

Hann, M.M., Keserü, G.M., 2012. Finding the sweet spot: the role of nature and nurture in medicinal chemistry. Nat Rev Drug Discov 11, 355-365.

Haraldsson, A.-K., Veide, J., Andlid, T., Alminger, M.L., Sandberg, A.-S., 2005. Degradation of phytate by high-phytase Saccharomyces cerevisiae strains during simulated gastrointestinal digestion. J. Agric. Food Chem. 53, 5438-5444.

Hendeles, L., Wubbena, P., Weinberger, M., 1984. Food-induced dose dumping of once-a-day theophylline. Lancet 2, 1471.

Home | IMI - Innovative Medicines Initiative [WWW Document], 2013. URL http://www.imi.europa.eu/\#\&panel1-2

ICH Expert Working Group, 2009. ICH Harmonised Tripartite Guideline, Pharmaceutical Development Q8 (R2), Current Step 4 Version.

Ishii, K., Saitou, Y., Yamada, R., Itai, S., Nemoto, M., 1996. Novel approach for determination of correlation between in vivo and in vitro dissolution using the optimization technique. Chem. Pharm. Bull. 44, 1550-1555. 
Jantratid, E., De Maio, V., Ronda, E., Mattavelli, V., Vertzoni, M., Dressman, J.B., 2009. Application of biorelevant dissolution tests to the prediction of in vivo performance of diclofenac sodium from an oral modified-release pellet dosage form. Eur J Pharm Sci 37, 434-441.

Jantratid, E., Janssen, N., Chokshi, H., Tang, K., Dressman, J.B., 2008a. Designing biorelevant dissolution tests for lipid formulations: case example--lipid suspension of RZ-50. Eur J Pharm Biopharm 69, 776-785.

Jantratid, E., Janssen, N., Reppas, C., Dressman, J.B., 2008b. Dissolution media simulating conditions in the proximal human gastrointestinal tract: an update. Pharm. Res. 25, 16631676.

Kakhi, M., 2009. Classification of the flow regimes in the flow-through cell. Eur J Pharm Sci 37, 531-544.

Kalantzi, L., Goumas, K., Kalioras, V., Abrahamsson, B., Dressman, J.B., Reppas, C., 2006. Characterization of the human upper gastrointestinal contents under conditions simulating bioavailability/bioequivalence studies. Pharm. Res. 23, 165-176.

Kalantzi, L., Page, R., Nicolaides, E., Digenis, G., Reppas, C., 2008. In vitro methods can forecast the effects of intragastric residence on dosage form performance. Eur J Pharm Sci 33, $445-451$.

Kalantzi, L., Polentarutti, B., Albery, T., Laitmer, D., Abrahamsson, B., Dressman, J., Reppas, C., 2005. The delayed dissolution of paracetamol products in the canine fed stomach can be predicted in vitro but it does not affect the onset of plasma levels. Int J Pharm 296, 8793.

Kataoka, M., Masaoka, Y., Sakuma, S., Yamashita, S., 2006. Effect of food intake on the oral absorption of poorly water-soluble drugs: in vitro assessment of drug dissolution and permeation assay system. J Pharm Sci 95, 2051-2061.

Kataoka, M., Masaoka, Y., Yamazaki, Y., Sakane, T., Sezaki, H., Yamashita, S., 2003. In vitro system to evaluate oral absorption of poorly water-soluble drugs: simultaneous analysis on dissolution and permeation of drugs. Pharm. Res. 20, 1674-1680.

Kataoka, M., Sugano, K., da Costa Mathews, C., Wong, J.W., Jones, K.L., Masaoka, Y., Sakuma, S., Yamashita, S., 2012. Application of dissolution/permeation system for evaluation of formulation effect on oral absorption of poorly water-soluble drugs in drug development. Pharm. Res. 29, 1485-1494.

Kataoka, M., Yokoyama, T., Masaoka, Y., Sakuma, S., Yamashita, S., 2011. Estimation of Pglycoprotein-mediated efflux in the oral absorption of P-gp substrate drugs from simultaneous analysis of drug dissolution and permeation. Eur J Pharm Sci 44, 544-551.

Kaukonen, A.M., Boyd, B.J., Charman, W.N., Porter, C.J.H., 2004. Drug solubilization behavior during in vitro digestion of suspension formulations of poorly water-soluble drugs in triglyceride lipids. Pharm. Res. 21, 254-260. 
Kelly, K., O’Mahony, B., Lindsay, B., Jones, T., Grattan, T.J., Rostami-Hodjegan, A., Stevens, H.N.E., Wilson, C.G., 2003. Comparison of the rates of disintegration, gastric emptying, and drug absorption following administration of a new and a conventional paracetamol formulation, using gamma scintigraphy. Pharm. Res. 20, 1668-1673.

Khamanga, S.M., Walker, R.B., 2006. Evaluation of rate of swelling and erosion of verapamil (VRP) sustained-release matrix tablets. Drug Dev Ind Pharm 32, 1139-1148.

Klein, S., 2009. Predicting food effects on drug release from extended-release oral dosage forms containing a narrow therapeutic index drug.

Klein, S., Dressman, J., 2006. Comparison of Drug Release From Metoprolol Modified Release Dosage.pdf Tutorial- Metoprolol. Dissolution Technologies 13, 6-12.

Klein, S., Garbacz, G., Pišlar, M., Locatelli, I., Liu, C., Weitschies, W., Siegmund, W., Mrhar, A., Bogataj, M., 2013. The role of individual gastric emptying of pellets in the prediction of diclofenac in vivo dissolution. J Control Release.

Klein, S., Rudolph, M.W., Skalsky, B., Petereit, H.-U., Dressman, J.B., 2008. Use of the BioDis to generate a physiologically relevant IVIVC. J Control Release 130, 216-219.

Klein, S., Shah, V.P., 2008. A standardized mini paddle apparatus as an alternative to the standard paddle. AAPS PharmSciTech 9, 1179-1184.

Klein, S., Stein, J., Dressman, J., 2005. Site-specific delivery of anti-inflammatory drugs in the gastrointestinal tract: an in-vitro release model. J. Pharm. Pharmacol. 57, 709-719.

Kloefer, B., van Hoogevest, P., Moloney, R., Kuentz, M., Mathew, L.S.L., Dressman, J., 2010. Study of a standardized taurocholate-lecithin powder for preparing the biorelevant media FaSSIF and FeSSIF, in: SIF Powder References: How It's Been Used to Make Biorelevant Media. Dissolution Technologies, pp. 6-13.

Kobayashi, M., Sada, N., Sugawara, M., Iseki, K., Miyazaki, K., 2001. Development of a new system for prediction of drug absorption that takes into account drug dissolution and $\mathrm{pH}$ change in the gastro-intestinal tract. Int J Pharm 221, 87-94.

Kola, I., 2008. The state of innovation in drug development. Clin. Pharmacol. Ther. 83, 227230.

Kola, I., Landis, J., 2004. Can the pharmaceutical industry reduce attrition rates? Nat Rev Drug Discov 3, 711-715.

Kong, F., Singh, R.P., 2010. A human gastric simulator (HGS) to study food digestion in human stomach. J. Food Sci. 75, E627-635.

Kostewicz, E.S., Wunderlich, M., Brauns, U., Becker, R., Bock, T., Dressman, J.B., 2004. Predicting the precipitation of poorly soluble weak bases upon entry in the small intestine. $\mathrm{J}$. Pharm. Pharmacol. 56, 43-51.

Krul, C., Luiten-Schuite, A., Baandagger, R., Verhagen, H., Mohn, G., Feron, V., Havenaar, R., 2000. Application of a dynamic in vitro gastrointestinal tract model to study the availability of food mutagens, using heterocyclic aromatic amines as model compounds. Food Chem. Toxicol. 38, 783-792. 
Larsen, A., Holm, R., Pedersen, M.L., Müllertz, A., 2008. Lipid-based formulations for danazol containing a digestible surfactant, Labrafil M2125CS: in vivo bioavailability and dynamic in vitro lipolysis. Pharm. Res. 25, 2769-2777.

Larsen, A.T., Sassene, P., Müllertz, A., 2011. In vitro lipolysis models as a tool for the characterization of oral lipid and surfactant based drug delivery systems. Int J Pharm 417, $245-255$.

Li, J., Yang, L., Ferguson, S.M., Hudson, T.J., Watanabe, S., Katsuma, M., Fix, J.A., 2002. In vitro evaluation of dissolution behavior for a colon-specific drug delivery system (CODES) in multi-pH media using United States Pharmacopeia apparatus II and III. AAPS PharmSciTech 3, E33.

Li, Y., McClements, D.J., 2011. Inhibition of lipase-catalyzed hydrolysis of emulsified triglyceride oils by low-molecular weight surfactants under simulated gastrointestinal conditions. Eur J Pharm Biopharm 79, 423-431.

Lila, M.A., Ribnicky, D.M., Rojo, L.E., Rojas-Silva, P., Oren, A., Havenaar, R., Janle, E.M., Raskin, I., Yousef, G.G., Grace, M.H., 2011. Complementary Approaches To Gauge the Bioavailability and Distribution of Ingested Berry Polyphenolics. J. Agric. Food Chem.

Lindahl, A., Ungell, A.L., Knutson, L., Lennernäs, H., 1997. Characterization of fluids from the stomach and proximal jejunum in men and women. Pharm. Res. 14, 497-502.

Lindfors, L., Forssén, S., Westergren, J., Olsson, U., 2008. Nucleation and crystal growth in supersaturated solutions of a model drug. J Colloid Interface Sci 325, 404-413.

Lobell, M., Hendrix, M., Hinzen, B., Keldenich, J., Meier, H., Schmeck, C., Schohe-Loop, R., Wunberg, T., Hillisch, A., 2006. In silico ADMET traffic lights as a tool for the prioritization of HTS hits. ChemMedChem 1, 1229-1236.

Loftsson, T., Brewster, M.E., 2011. Pharmaceutical applications of cyclodextrins: effects on drug permeation through biological membranes. J. Pharm. Pharmacol. 63, 1119-1135.

Malinowski, H., Marroum, P., Uppoor, V.R., Gillespie, W., Ahn, H.Y., Lockwood, P., Henderson, J., Baweja, R., Hossain, M., Fleischer, N., Tillman, L., Hussain, A., Shah, V., Dorantes, A., Zhu, R., Sun, H., Kumi, K., Machado, S., Tammara, V., Ong-Chen, T.E., Mahayni, H., Lesko, L., Williams, R., 1997. Draft guidance for industry extended-release solid oral dosage forms. Development, evaluation and application of in vitro-in vivo correlations. Adv. Exp. Med. Biol. 423, 269-288.

Mann, J., Pygall, S., 2012. A Formulation Case Study Comparing the Dynamic Gastric Model with Conventional Dissolution Methods" - Dissolution Technologies, Vol 19, Issue 4, 14-19 http://www.dissolutiontech.com/DTresour/201211Articles/DT201211_A02.pdf. Dissolution Technologies 14-19.

Marciani, L., Gowland, P.A., Fillery-Travis, A., Manoj, P., Wright, J., Smith, A., Young, P., Moore, R., Spiller, R.C., 2001a. Assessment of antral grinding of a model solid meal with echo-planar imaging. Am. J. Physiol. Gastrointest. Liver Physiol. 280, G844-849. 
Marciani, L., Gowland, P.A., Spiller, R.C., Manoj, P., Moore, R.J., Young, P., Al-Sahab, S., Bush, D., Wright, J., Fillery-Travis, A.J., 2000. Gastric response to increased meal viscosity assessed by echo-planar magnetic resonance imaging in humans. J. Nutr. 130, 122-127.

Marciani, L., Gowland, P.A., Spiller, R.C., Manoj, P., Moore, R.J., Young, P., Fillery-Travis, A.J., 2001b. Effect of meal viscosity and nutrients on satiety, intragastric dilution, and emptying assessed by MRI. Am. J. Physiol. Gastrointest. Liver Physiol. 280, G1227-1233.

Marciani, L., Wickham, M., Hills, B. p., Wright, J., Bush, D., Faulks, R., Fillery-Travis, A., Spiller, R. c., Gowl, P.A., 2004. Intragastric Oil-in-Water Emulsion Fat Fraction Measured Using Inversion Recovery Echo-Planar Magnetic Resonance Imaging. Journal of Food Science 69, E290-E296.

McAllister, M., 2010. Dynamic dissolution: a step closer to predictive dissolution testing? Mol. Pharm. 7, 1374-1387.

McCarthy, L.G., Kosiol, C., Healy, A.M., Bradley, G., Sexton, J.C., Corrigan, O.I., 2003. Simulating the hydrodynamic conditions in the United States Pharmacopeia paddle dissolution apparatus. AAPS PharmSciTech 4, E22.

McMahon, N., Crane, M., Ruskin, H.J., Crane, L., 2007. The importance of boundary conditions in the simulation of dissolution in the USP dissolution apparatus. Simulation Modelling Practice and Theory 15, 247-255.

Measuring the return from Pharmaceutical innovation 2012 - Deloitte UK Centre for Health Solutions | Deloitte UK [WWW Document], 2013. URL http://www.twylah.com/Deloitte/tweets/275996178636754945

Mellaerts, R., Mols, R., Kayaert, P., Annaert, P., Van Humbeeck, J., Van den Mooter, G., Martens, J.A., Augustijns, P., 2008. Ordered mesoporous silica induces pH-independent supersaturation of the basic low solubility compound itraconazole resulting in enhanced transepithelial transport. Int J Pharm 357, 169-179.

Mercuri, A., Passalacqua, A., Wickham, M.S.J., Faulks, R.M., Craig, D.Q.M., Barker, S.A., 2011. The effect of composition and gastric conditions on the self-emulsification process of ibuprofen-loaded self-emulsifying drug delivery systems: a microscopic and dynamic gastric model study. Pharm. Res. 28, 1540-1551.

Miled, N., Canaan, S., Dupuis, L., Roussel, A., Rivière, M., Carrière, F., de Caro, A., Cambillau, C., Verger, R., 2000. Digestive lipases: from three-dimensional structure to physiology. Biochimie 82, 973-986.

Miller, J.M., Beig, A., Krieg, B.J., Carr, R.A., Borchardt, T.B., Amidon, G.E., Amidon, G.L., Dahan, A., 2011. The solubility-permeability interplay: mechanistic modeling and predictive application of the impact of micellar solubilization on intestinal permeation. Mol. Pharm. 8, $1848-1856$.

Miller, J.M., Dahan, A., 2012. Predicting the solubility-permeability interplay when using cyclodextrins in solubility-enabling formulations: model validation. Int J Pharm 430, 388391. 
Minekus, M., Jelier, M., Xiao, J.-Z., Kondo, S., Iwatsuki, K., Kokubo, S., Bos, M., Dunnewind, B., Havenaar, R., 2005. Effect of partially hydrolyzed guar gum (PHGG) on the bioaccessibility of fat and cholesterol. Biosci. Biotechnol. Biochem. 69, 932-938.

Minekus, M., Marteau, P., Havenaar, R., Huis in 't Veld, J.H.J., 1995. A multicompartmental dynamic computer-controlled model simulating the stomach and small intestine. ATLA. Alternatives to laboratory animals 23, 197-209.

Minekus, M., Smeets-Peeters, M., Bernalier, A., Marol-Bonnin, S., Havenaar, R., Marteau, P., Alric, M., Fonty, G., Huis in't Veld, J.H., 1999. A computer-controlled system to simulate conditions of the large intestine with peristaltic mixing, water absorption and absorption of fermentation products. Appl. Microbiol. Biotechnol. 53, 108-114.

Miners, J.O., Knights, K.M., Houston, J.B., Mackenzie, P.I., 2006. In vitro-in vivo correlation for drugs and other compounds eliminated by glucuronidation in humans: pitfalls and promises. Biochem. Pharmacol. 71, 1531-1539.

Mirza, T., Joshi, Y., Vivilecchia, R., 2013. Evaluation of dissolution hydrodynamics in the USP, Peak $^{\mathrm{TM}}$ and flat-bottom vessels using different solubility drugs | ResearchGate [WWW Document].

URL http://www.researchgate.net/publication/228485312_Evaluation_of_dissolution_hydrodynami cs_in_the_USP_Peak_and_flat-bottom_vessels_using_different_solubility_drugs

Missaghi, S., Fassihi, R., 2005. Release characterization of dimenhydrinate from an eroding and swelling matrix: selection of appropriate dissolution apparatus. Int J Pharm 293, 35-42.

Mohsin, K., 2012. Design of lipid-based formulations for oral administration of poorly watersoluble drug fenofibrate: effects of digestion. AAPS PharmSciTech 13, 637-646.

Moore, J.G., Christian, P.E., Brown, J.A., Brophy, C., Datz, F., Taylor, A., Alazraki, N., 1984. Influence of meal weight and caloric content on gastric emptying of meals in man. Dig. Dis. Sci. 29, 513-519.

Moos, W.H., Green, G.D., Pavia, M.R., 1993. Chapter 33. Recent Advances in the Generation of Molecular Diversity, in: James A. Bristol (Ed.), Annual Reports in Medicinal Chemistry. Academic Press, pp. 315-324.

Motz, S.A., Schaefer, U.F., Balbach, S., Eichinger, T., Lehr, C.-M., 2007. Permeability assessment for solid oral drug formulations based on Caco-2 monolayer in combination with a flow through dissolution cell. Eur J Pharm Biopharm 66, 286-295.

Naylor, T.A., Connolly, P.C., Martini, L.G., Elder, D.P., Minekus, M., Havenaar, R., Zeijdner, E., 2006. Use of a gastro-intestinal model and GastroPLUS ${ }^{\mathrm{TM}}$ for the prediction of in vivo performance. JOURNAL OF APPLIED THERAPEUTIC RESEARCH 6, 15.

New Drug Approvals [WWW Document], 2013. URL http://www.drugs.com/newdrugs.html

Nicolaides, E., Symillides, M., Dressman, J.B., Reppas, C., 2001. Biorelevant dissolution testing to predict the plasma profile of lipophilic drugs after oral administration. Pharm. Res. $18,380-388$. 
Nury, S., Piéroni, G., Rivière, C., Gargouri, Y., Bois, A., Verger, R., 1987. Lipase kinetics at the triacylglycerol-water interface using surface tension measurements. Chem. Phys. Lipids $45,27-37$.

Okumu, A., DiMaso, M., Löbenberg, R., 2008. Dynamic dissolution testing to establish in vitro/in vivo correlations for montelukast sodium, a poorly soluble drug. Pharm. Res. 25, 2778-2785.

Okumu, A., DiMaso, M., Löbenberg, R., 2009. Computer simulations using GastroPlus to justify a biowaiver for etoricoxib solid oral drug products. Eur J Pharm Biopharm 72, 91-98.

Pang, K.S., Liu, L., Sun, H., 2006. Interaction of Drug Transporters with Excipients, in: Wasan, K.M. (Ed.), Role of Lipid Excipients in Modifying Oral and Parenteral Drug Delivery. John Wiley \& Sons, Inc., pp. 1-31.

Patel, D.V., Gordon, E.M., 1996. Applications of small-molecule combinatorial chemistry to drug discovery. Drug Discovery Today 1, 134-144.

Perez de la Cruz Moreno, M., Oth, M., Deferme, S., Lammert, F., Tack, J., Dressman, J., Augustijns, P., 2006. Characterization of fasted-state human intestinal fluids collected from duodenum and jejunum. J. Pharm. Pharmacol. 58, 1079-1089.

Pharmacopeial Forum, 1988. Stimuli to the revision process: In vitro/in vivo correlation for extended release dosage forms.

PMA Joint Committee on Bioavailability,1985. The role of dissolution testing in drug quality, bioavailability, and bioequivalence testing - NeLM, Pharmaceutical Technology.

Polli, J.E., 2008. In vitro studies are sometimes better than conventional human pharmacokinetic in vivo studies in assessing bioequivalence of immediate-release solid oral dosage forms. AAPS J 10, 289-299.

Polster, C.S., Atassi, F., Wu, S.-J., Sperry, D.C., 2010. Use of artificial stomach-duodenum model for investigation of dosing fluid effect on clinical trial variability. Mol. Pharm. 7, $1533-1538$.

Porter, C.J.H., Kaukonen, A.M., Boyd, B.J., Edwards, G.A., Charman, W.N., 2004. Susceptibility to lipase-mediated digestion reduces the oral bioavailability of danazol after administration as a medium-chain lipid-based microemulsion formulation. Pharm. Res. 21, $1405-1412$.

Porter, C.J.H., Trevaskis, N.L., Charman, W.N., 2007. Lipids and lipid-based formulations: optimizing the oral delivery of lipophilic drugs. Nat Rev Drug Discov 6, 231-248.

ProDigest - Gastrointestinal Expertise [WWW Document], 2013. URL http://www.prodigest.eu/en/home

Psachoulias, D., Vertzoni, M., Butler, J., Busby, D., Symillides, M., Dressman, J., Reppas, C., 2012. An in vitro methodology for forecasting luminal concentrations and precipitation of highly permeable lipophilic weak bases in the fasted upper small intestine. Pharm. Res. 29, 3486-3498. 
Qiu, Y., Chen, Y., Zhang, G.G.Z., Liu, L., Porter, W., 2009. Developing Solid Oral Dosage Forms: Pharmaceutical Theory \& Practice. Academic Press.

Radwan, A., Amidon, G.L., Langguth, P., 2012. Mechanistic investigation of food effect on disintegration and dissolution of BCS class III compound solid formulations: the importance of viscosity. Biopharm Drug Dispos 33, 403-416.

Ramos Pezzini, B., Gomes Ferraz, H., 2009. Bio-dis and the paddle dissolution apparatuses applied to the release characterization of ketoprofen from hypromellose matrices. AAPS PharmSciTech 10, 763-771.

Reis, P.M., Raab, T.W., Chuat, J.Y., Leser, M.E., Miller, R., Watzke, H.J., Holmberg, K., 2008. Influence of Surfactants on Lipase Fat Digestion in a Model Gastro-intestinal System. Food Biophys 3, 370-381.

Rohrs, B.R., Burch-Clark, D.L., Witt, M.J., Stelzer, D.J., 1995. USP dissolution apparatus 3 (reciprocating cylinder): instrument parameter effects on drug release from sustained release formulations. J Pharm Sci 84, 922-926.

Roman, M.J., Burri, B.J., Singh, R.P., 2012. Release and bioaccessibility of $\beta$-carotene from fortified almond butter during in vitro digestion. J. Agric. Food Chem. 60, 9659-9666.

Rostami-Hodjegan, A., Shiran, M.R., Ayesh, R., Grattan, T.J., Burnett, I., Darby-Dowman, A., Tucker, G.T., 2002a. A new rapidly absorbed paracetamol tablet containing sodium bicarbonate. I. A four-way crossover study to compare the concentration-time profile of paracetamol from the new paracetamol/sodium bicarbonate tablet and a conventional paracetamol tablet in fed and fasted volunteers. Drug Dev Ind Pharm 28, 523-531.

Rostami-Hodjegan, A., Shiran, M.R., Tucker, G.T., Conway, B.R., Irwin, W.J., Shaw, L.R., Grattan, T.J., 2002b. A new rapidly absorbed paracetamol tablet containing sodium bicarbonate. II. Dissolution studies and in vitro/in vivo correlation. Drug Dev Ind Pharm 28, 533-543.

Rygnestad, T., Zahlsen, K., Samdal, F.A., 2000. Absorption of effervescent paracetamol tablets relative to ordinary paracetamol tablets in healthy volunteers. Eur. J. Clin. Pharmacol. $56,141-143$.

Sandberg, A., Abrahamsson, B., Sjörgen, J., 1991. Influence of dissolution rate on the extent and rate of bioavailability of metoprolol. International Journal of Pharmaceutics 1-3, 167177.

Sassene, P.J., Knopp, M.M., Hesselkilde, J.Z., Koradia, V., Larsen, A., Rades, T., Müllertz, A., 2010. Precipitation of a poorly soluble model drug during in vitro lipolysis: characterization and dissolution of the precipitate. J Pharm Sci 99, 4982-4991.

Schiller, C., Fröhlich, C.-P., Giessmann, T., Siegmund, W., Mönnikes, H., Hosten, N., Weitschies, W., 2005. Intestinal fluid volumes and transit of dosage forms as assessed by magnetic resonance imaging. Aliment. Pharmacol. Ther. 22, 971-979. 
Scholz, A., Abrahamsson, B., Diebold, S.M., Kostewicz, E., Polentarutti, B.I., Ungell, A.-L., Dressman, J.B., 2002. Influence of hydrodynamics and particle size on the absorption of felodipine in labradors. Pharm. Res. 19, 42-46.

Scholz, A., Kostewicz, E., Abrahamsson, B., Dressman, J.B., 2003. Can the USP paddle method be used to represent in-vivo hydrodynamics? J. Pharm. Pharmacol. 55, 443-451.

Shah, V.P., Gurbarg, M., Noory, A., Dighe, S., Skelly, J.P., 1992. Influence of higher rates of agitation on release patterns of immediate-release drug products. J Pharm Sci 81, 500-503.

Shameem, M., Katori, N., Aoyagi, N., Kojima, S., 1995. Oral solid controlled release dosage forms: role of GI-mechanical destructive forces and colonic release in drug absorption under fasted and fed conditions in humans. Pharm. Res. 12, 1049-1054.

Shaw, T.R., Raymond, K., Howard, M.R., Hamer, J., 1973. Therapeutic non-equivalence of digoxin tablets in the United Kingdom: correlation with tablet dissolution rate. Br Med J 4, 763-766.

Sheiner, L.B., 1997. Learning versus confirming in clinical drug development. Clin. Pharmacol. Ther. 61, 275-291.

Sheng, J.J., Sirois, P.J., Dressman, J.B., Amidon, G.L., 2008. Particle diffusional layer thickness in a USP dissolution apparatus II: a combined function of particle size and paddle speed. J Pharm Sci 97, 4815-4829.

Shi, Y., Gao, P., Gong, Y., Ping, H., 2010. Application of a biphasic test for characterization of in vitro drug release of immediate release formulations of celecoxib and its relevance to in vivo absorption. Mol. Pharm. 7, 1458-1465.

Shiko, G., Gladden, L.F., Sederman, A.J., Connolly, P.C., Butler, J.M., 2011. MRI studies of the hydrodynamics in a USP 4 dissolution testing cell. J Pharm Sci 100, 976-991.

Shono, Y., Jantratid, E., Janssen, N., Kesisoglou, F., Mao, Y., Vertzoni, M., Reppas, C., Dressman, J.B., 2009. Prediction of food effects on the absorption of celecoxib based on biorelevant dissolution testing coupled with physiologically based pharmacokinetic modeling. Eur J Pharm Biopharm 73, 107-114.

Skelley, J.P., Amidon, G.L., Barr, W.H., Benet, L.Z., Carter, J.E., Robinson, J.R., Shah, V.P., Yacobi, A., 1990. Report of the workshop on in vitro and in vivo testing and correlation for oral controlled/modified-release dosage forms. J Pharm Sci 79, 849-854.

Souliman, S., Beyssac, E., Cardot, J.-M., Denis, S., Alric, M., 2007. Investigation of the biopharmaceutical behavior of theophylline hydrophilic matrix tablets using USP methods and an artificial digestive system. Drug Dev Ind Pharm 33, 475-483.

Souliman, S., Blanquet, S., Beyssac, E., Cardot, J.-M., 2006. A level A in vitro/in vivo correlation in fasted and fed states using different methods: applied to solid immediate release oral dosage form. Eur J Pharm Sci 27, 72-79. 
Sugawara, M., Kadomura, S., He, X., Takekuma, Y., Kohri, N., Miyazaki, K., 2005. The use of an in vitro dissolution and absorption system to evaluate oral absorption of two weak bases in $\mathrm{pH}$-independent controlled-release formulations. Eur J Pharm Sci 26, 1-8.

Sunesen, V.H., Pedersen, B.L., Kristensen, H.G., Müllertz, A., 2005. In vivo in vitro correlations for a poorly soluble drug, danazol, using the flow-through dissolution method with biorelevant dissolution media. Eur J Pharm Sci 24, 305-313.

Takano, R., Kataoka, M., Yamashita, S., 2012. Integrating drug permeability with dissolution profile to develop IVIVC. Biopharm Drug Dispos 33, 354-365.

Takano, R., Takata, N., Saito, R., Furumoto, K., Higo, S., Hayashi, Y., Machida, M., Aso, Y., Yamashita, S., 2010. Quantitative analysis of the effect of supersaturation on in vivo drug absorption. Mol. Pharm. 7, 1431-1440.

Tannergren, C., Bergendal, A., Lennernäs, H., Abrahamsson, B., 2009. Toward an increased understanding of the barriers to colonic drug absorption in humans: implications for early controlled release candidate assessment. Mol. Pharm. 6, 60-73.

Tenjarla, S., Romasanta, V., Zeijdner, E., Villa, R., Moro, L., 2007. Release of 5aminosalicylate from an MMX mesalamine tablet during transit through a simulated gastrointestinal tract system. Adv Ther 24, 826-840.

The European Pharmacopoeia, 2011. European Directorate for the Quality of Medicines and HealthCare, Council of Europe, 7th ed. Strasbourg, France.

The United States Pharmacopeial Convention, Inc., 2013. The United States Pharmacopeia The National Formulary. USP 30/ NF 25, Rockville, MD.

Thomas, N., Holm, R., Müllertz, A., Rades, T., 2012a. In vitro and in vivo performance of novel supersaturated self-nanoemulsifying drug delivery systems (super-SNEDDS). J Control Release 160, 25-32.

Thomas, N., Holm, R., Rades, T., Müllertz, A., 2012b. Characterising lipid lipolysis and its implication in lipid-based formulation development. AAPS J 14, 860-871.

Thybo, P., Pedersen, B.L., Hovgaard, L., Holm, R., Mullertz, A., 2008. Characterization and physical stability of spray dried solid dispersions of probucol and PVP-K30. Pharm Dev Technol 13, 375-386.

Tønsberg, H., Holm, R., Bisgaard, J., Jacobsen, J., Müllertz, A., 2010. Effects of polysorbate 80 on the in-vitro precipitation and oral bioavailability of halofantrine from polyethylene glycol 400 formulations in rats. J. Pharm. Pharmacol. 62, 63-70.

United States Pharmacopoeia Convention, 2012. United States Pharmacopoeia 35 - National Formulary 30.

US Pharmacopoeia, 2011. U.S. Pharmacopoeia National Formulary 2011: USP 34 NF 29.

Van Speybroeck, M., Mols, R., Mellaerts, R., Thi, T.D., Martens, J.A., Van Humbeeck, J., Annaert, P., Van den Mooter, G., Augustijns, P., 2010. Combined use of ordered mesoporous 
silica and precipitation inhibitors for improved oral absorption of the poorly soluble weak base itraconazole. Eur J Pharm Biopharm 75, 354-365.

Vardakou, M., Mercuri, A., Barker, S.A., Craig, D.Q.M., Faulks, R.M., Wickham, M.S.J., 2011a. Achieving antral grinding forces in biorelevant in vitro models: comparing the USP dissolution apparatus II and the dynamic gastric model with human in vivo data. AAPS PharmSciTech 12, 620-626.

Vardakou, M., Mercuri, A., Naylor, T.A., Rizzo, D., Butler, J.M., Connolly, P.C., Wickham, M.S.J., Faulks, R.M., 2011b. Predicting the human in vivo performance of different oral capsule shell types using a novel in vitro dynamic gastric model. Int J Pharm 419, 192-199.

Vatier, J., Célice-Pingaud, C., Farinotti, R., 1998. A computerized artificial stomach model to assess sodium alginate-induced $\mathrm{pH}$ gradient. International Journal of Pharmaceutics 163, 225229.

Vatier, J., Harman, A., Castela, N., Droy-Lefaix, M.T., Farinotti, R., 1994. Interactions of cimetidine and ranitidine with aluminum-containing antacids and a clay-containing gastricprotective drug in an "artificial stomach-duodenum" model. J Pharm Sci 83, 962-966.

Vertzoni, M., Diakidou, A., Chatzilias, M., Söderlind, E., Abrahamsson, B., Dressman, J.B., Reppas, C., 2010. Biorelevant media to simulate fluids in the ascending colon of humans and their usefulness in predicting intracolonic drug solubility. Pharm. Res. 27, 2187-2196.

Vertzoni, M., Dressman, J., Butler, J., Hempenstall, J., Reppas, C., 2005. Simulation of fasting gastric conditions and its importance for the in vivo dissolution of lipophilic compounds. Eur J Pharm Biopharm 60, 413-417.

Vertzoni, M., Iliadis, A., Nicolaides, E., Symillides, M., Reppas, C., 2006. Orally administered drug products: dissolution data analysis with a view to IVIVC in Pharmaceutical Dissolution Testing, Dressman J.B. and Kraemer J. (editors). New York, USA, pp. 229-249.

Vertzoni, M., Markopoulos, C., Symillides, M., Goumas, C., Imanidis, G., Reppas, C., 2012. Luminal lipid phases after administration of a triglyceride solution of danazol in the fed state and their contribution to the flux of danazol across Caco-2 cell monolayers. Mol. Pharm. 9, 1189-1198.

Verwei, M., Arkbåge, K., Havenaar, R., van den Berg, H., Witthöft, C., Schaafsma, G., 2003. Folic acid and 5-methyltetrahydrofolate in fortified milk are bioaccessible as determined in a dynamic in vitro gastrointestinal model. J. Nutr. 133, 2377-2383.

Verwei, M., Freidig, A.P., Havenaar, R., Groten, J.P., 2006. Predicted serum folate concentrations based on in vitro studies and kinetic modeling are consistent with measured folate concentrations in humans. J. Nutr. 136, 3074-3078.

Vieth, M., Siegel, M.G., Higgs, R.E., Watson, I.A., Robertson, D.H., Savin, K.A., Durst, G.L., Hipskind, P.A., 2004. Characteristic physical properties and structural fragments of marketed oral drugs. J. Med. Chem. 47, 224-232. 
Wei, H., Löbenberg, R., 2006. Biorelevant dissolution media as a predictive tool for glyburide a class II drug. Eur J Pharm Sci 29, 45-52.

Weitschies, W., Friedrich, C., Wedemeyer, R.S., Schmidtmann, M., Kosch, O., Kinzig, M., Trahms, L., Sörgel, F., Siegmund, W., Horkovics-Kovats, S., Schwarz, F., Raneburger, J., Mönnikes, H., 2008. Bioavailability of amoxicillin and clavulanic acid from extended release tablets depends on intragastric tablet deposition and gastric emptying. Eur J Pharm Biopharm 70, 641-648.

Weitschies, W., Karaus, M., Cordini, D., Trahms, L., Breitkreutz, J., Semmler, W., 2001. Magnetic marker monitoring of disintegrating capsules. Eur J Pharm Sci 13, 411-416.

Weitschies, W., Wedemeyer, R.-S., Kosch, O., Fach, K., Nagel, S., Söderlind, E., Trahms, L., Abrahamsson, B., Mönnikes, H., 2005. Impact of the intragastric location of extended release tablets on food interactions. J Control Release 108, 375-385.

Welling, P.G., Tse, F.L.S., Dighe, S.V., 1991. Pharmaceutical bioequivalence / edited by Peter G. Welling, Francis L.S. Tse, Shrikant V. Dighe, Drugs and the pharmaceutical sciences; v. 48. Dekker, New York:

Wickham, Faulks, Mann, Mandalari, 2012. The Design, Operation, and Application of a Dynamic Gastric Model. Dissolution Technologies 15-22.

Wickham, M., Faulks, R., Mills, C., 2009. In vitro digestion methods for assessing the effect of food structure on allergen breakdown. Mol Nutr Food Res 53, 952-958.

Williams, H.D., Anby, M.U., Sassene, P., Kleberg, K., Bakala-N'Goma, J.-C., Calderone, M., Jannin, V., Igonin, A., Partheil, A., Marchaud, D., Jule, E., Vertommen, J., Maio, M., Blundell, R., Benameur, H., Carrière, F., Müllertz, A., Pouton, C.W., Porter, C.J.H., 2012a. Toward the establishment of standardized in vitro tests for lipid-based formulations. 2. The effect of bile salt concentration and drug loading on the performance of type I, II, IIIA, IIIB, and IV formulations during in vitro digestion. Mol. Pharm. 9, 3286-3300.

Williams, H.D., Sassene, P., Kleberg, K., Bakala-N'Goma, J.-C., Calderone, M., Jannin, V., Igonin, A., Partheil, A., Marchaud, D., Jule, E., Vertommen, J., Maio, M., Blundell, R., Benameur, H., Carrière, F., Müllertz, A., Porter, C.J.H., Pouton, C.W., 2012b. Toward the establishment of standardized in vitro tests for lipid-based formulations, part 1: method parameterization and comparison of in vitro digestion profiles across a range of representative formulations. J Pharm Sci 101, 3360-3380.

Williams, H.D., Trevaskis, N.L., Charman, S.A., Shanker, R.M., Charman, W.N., Pouton, C.W., Porter, C.J.H., 2013. Strategies to address low drug solubility in discovery and development. Pharmacol. Rev. 65, 315-499.

Wu, Y., Kildsig, D.O., Ghaly, E.S., 2004. Effect of hydrodynamic environment on tablets dissolution rate. Pharm Dev Technol 9, 25-37.

Wulff-Pérez, M., de Vicente, J., Martín-Rodríguez, A., Gálvez-Ruiz, M.J., 2012. Controlling lipolysis through steric surfactants: new insights on the controlled degradation of submicron emulsions after oral and intravenous administration. Int J Pharm 423, 161-166. 
Yano, K., Masaoka, Y., Kataoka, M., Sakuma, S., Yamashita, S., 2010. Mechanisms of membrane transport of poorly soluble drugs: role of micelles in oral absorption processes. $\mathbf{J}$ Pharm Sci 99, 1336-1345.

Yeap, Y.Y., Trevaskis, N.L., Quach, T., Tso, P., Charman, W.N., Porter, C.J., 2013. Intestinal bile secretion promotes drug absorption from lipid colloidal phases via induction of supersaturation. Mol. Pharm., 10, 1874-1889.

Yu, L.X., 2008. Pharmaceutical quality by design: product and process development, understanding, and control. Pharm. Res. 25, 781-791.

Zangenberg, N.H., Müllertz, A., Kristensen, H.G., Hovgaard, L., 2001. A dynamic in vitro lipolysis model. II: Evaluation of the model. Eur J Pharm Sci 14, 237-244. 


\section{$9 \quad$ Figure Legends}

Figure 1: (A) Basket (USP 1) and (B) Paddle (USP 2) apparatus (Diebold, 2006), (C) Reciprocating cylinder apparatus (USP 3), and (D) Flow-through apparatus (USP 4) (upper panel: open-loop configuration; lower panel: closed-loop configuration).

Figure 2: Coning below paddle in the USP 2 apparatus

Figure 3: A schematic of a USP 4 flow through cell showing the main components, the direction of flow and the tablet position when placed horizontally and vertically. The dashed rectangles indicate the orientation of the MRI slices in Cartesian coordinates. (Reproduced from Shiko et al. 2011)

Figure 4: Transfer model for prediction of intestinal precipitation

Figure 5: Individual diclofenac plasma concentration profiles obtained after the administration of $100 \mathrm{mg}$ diclofenac ER tablets under fasting conditions $(\mathrm{n}=24)$. In the inset means and standard deviations are shown.

Figure 6: Schematic representation of dissolution stress test apparatus

Figure 7: An example of a lipolysis model (adapted from Porter et al., 2007)

Figure 8: TIM-1 system (A. stomach compartment; B. pyloric sphincter; C. duodenum compartment; D. peristaltic valve; E. jejunum compartment; F. peristaltic valve; G. ileum compartment; H. ileo-caecal sphincter; I. stomach secretion; J. duodenum secretion; K. jejunum/ileum secretion; L. pre-filter; M. semi-permeable membrane; N. filtrate pump; P. pH electrodes; Q. level sensors; R. temperature sensor; S. pressure sensor) 


\section{Tables}

Table 1: A comparison of the key features of complex 'GI'-like dissolution systems

\begin{tabular}{|c|c|c|c|c|}
\hline & & \multicolumn{3}{|c|}{ Complex 'Gl'-like dissolution systems } \\
\hline & $\begin{array}{l}\text { Physiological } \\
\text { parameter } \\
\text { simulated }\end{array}$ & $\begin{array}{l}\text { Artificial Stomach } \\
\text { Duodenum model }\end{array}$ & $\begin{array}{c}\text { IFR Dynamic Gastric } \\
\text { model }\end{array}$ & TNO TIM-1 \\
\hline \multirow{6}{*}{$\begin{array}{l}\text { Compositional } \\
\text { similarity to } \\
\text { lumenal fluids }\end{array}$} & Gastric volume & $\begin{array}{l}\text { Fasted state: } 50 \mathrm{~mL} \\
\text { of gastric fluid and } \\
50 \mathrm{~mL} \text { water; Fed } \\
\text { state: } 250 \mathrm{~mL}\end{array}$ & $800 \mathrm{~mL}$ & $150-300 \mathrm{~mL}$ \\
\hline & $\begin{array}{l}\text { Small intestinal } \\
\text { volume }\end{array}$ & duodenum $30 \mathrm{~mL}$ & & $\begin{array}{l}300 \mathrm{~mL} \text { (duodenum } 50 \mathrm{~mL} \text {, } \\
\text { jejunum } 125 \mathrm{~mL} \text {, ileum } 125 \\
\mathrm{~mL} \text { ) }\end{array}$ \\
\hline & $\mathrm{pH}$ & $\begin{array}{l}\text { Gastric pH: } 2 \text { or } 5.5 \\
\text { Duodenal pH: } 6.5\end{array}$ & $\begin{array}{l}\text { gastric } \mathrm{pH} \text { curve } \\
\text { depending on the } \\
\text { intake }\end{array}$ & $\begin{array}{l}\text { Stomach: } \mathrm{pH} \text { curve depending } \\
\text { on fasting / fed state and } \\
\text { type of food } \\
\text { Duodenum: } 5.9-6.4 \\
\text { Jejunum : } 6.4-6.6 \\
\text { Ileum: } 7.2-7.4\end{array}$ \\
\hline & Buffer system & $\begin{array}{l}\text { Gastric } \mathrm{pH} \text { adjusted } \\
\text { with } \mathrm{HCl} \text {. Duodenal } \\
\text { pH maintained with } \\
\text { phosphate buffer }\end{array}$ & $\begin{array}{l}\text { Gastric } \mathrm{pH} \text { controlled } \\
\text { with } \mathrm{HCl}\end{array}$ & $\begin{array}{l}\text { Gastric } \mathrm{pH} \text { controlled with } \\
\mathrm{HCl} \text {. Small intestinal } \mathrm{pH} \\
\text { controlled with bicarbonate } \\
\text { buffer. }\end{array}$ \\
\hline & Fed state simulation & $\begin{array}{l}\text { Buffer pH and } \\
\text { compositional } \\
\text { change only }\end{array}$ & $\begin{array}{l}\text { Whole food-pre- } \\
\text { masticated }\end{array}$ & $\begin{array}{l}\text { Liquid or solid food } \\
\text { Artificially masticated } \\
\text { Mixed with saliva }\end{array}$ \\
\hline & $\begin{array}{l}\text { Bile salt/enzyme } \\
\text { secretion }\end{array}$ & $\begin{array}{l}\text { No enzymes. } \\
\text { Addition of sodium } \\
\text { taurocholate in } \\
\text { duodenum }\end{array}$ & $\begin{array}{l}\text { Controlled secretion } \\
\text { of artificial gastric } \\
\text { juice with gastric } \\
\text { enzymes (pepsin, } \\
\text { lipase) }\end{array}$ & $\begin{array}{l}\text { Controlled secretion of } \\
\text { artificial saliva and gastric } \\
\text { juice with enzymes (amylase, } \\
\text { pepsin, lipase). Controlled } \\
\text { duodenal secretion of small } \\
\text { intestinal enzymes } \\
\text { (pancreatin) and complete } \\
\text { bile }\end{array}$ \\
\hline
\end{tabular}




\begin{tabular}{|c|c|c|c|c|}
\hline \multirow{3}{*}{$\begin{array}{l}\text { Physical } \\
\text { parameters }\end{array}$} & Gastric emptying & \begin{tabular}{|l} 
Programmable \\
gastric emptying \\
half-life.
\end{tabular} & $\begin{array}{l}\text { Programmable } \\
\text { gastric emptying } \\
\text { curve }\end{array}$ & $\begin{array}{l}\text { Programmable gastric } \\
\text { emptying curve, depending } \\
\text { on type and amount of food }\end{array}$ \\
\hline & $\begin{array}{l}\text { Dosage form } \\
\text { movement }\end{array}$ & $\begin{array}{l}\text { Transfer of } \\
\text { disintegrated } \\
\text { material only }\end{array}$ & $\begin{array}{l}\text { Single compartment } \\
\text { only }\end{array}$ & $\begin{array}{l}\text { Liquids, suspensions and } \\
\text { disintegrated material with } \\
\text { gastric emptying and } \\
\text { intestinal transit. } \\
\text { Non-disintegrated dosage } \\
\text { forms require manual } \\
\text { intervention to move } \\
\text { between compartments }\end{array}$ \\
\hline & $\begin{array}{l}\text { Simulation of } \\
\text { physiologically } \\
\text { relevant mechanical } \\
\text { stresses }\end{array}$ & None & $\begin{array}{l}\text { Simulation of gastric } \\
\text { body movements } \\
\text { with flexible wall. } \\
\text { Simulation of antral } \\
\text { mixing by } \\
\text { movements of an } \\
\text { elastic annulus }\end{array}$ & $\begin{array}{l}\text { Simulation of peristalsis } \\
\text { through pressure changes on } \\
\text { flexible wall. Hydrodynamic } \\
\text { shear and pressures }\end{array}$ \\
\hline \multirow{5}{*}{ Permeation step } & $\begin{array}{l}\text { Removal of } \\
\text { dissolved material } \\
\text { (absorption sink) }\end{array}$ & $\begin{array}{l}\text { Volume of duodenal } \\
\text { chamber maintained } \\
\text { by removal of } \\
\text { disintegrated and } \\
\text { dissolved material } \\
\text { and addition of fresh } \\
\text { buffer }\end{array}$ & $\begin{array}{l}\text { None, gastric } \\
\text { empyting of whole } \\
\text { contents }\end{array}$ & $\begin{array}{l}\text { Removal of dissolved material } \\
\text { and digested products across } \\
\text { a dialysis or filtration } \\
\text { membrane }\end{array}$ \\
\hline & $\begin{array}{l}\text { Simulation of } \\
\text { intestinal transport } \\
\text { (active \& passive) }\end{array}$ & None & None & None \\
\hline & $\begin{array}{l}\text { Gut wall } \\
\text { metabolism }\end{array}$ & None & None & None \\
\hline & $\begin{array}{l}\text { Mucosal } \\
\text { microenvironment }\end{array}$ & None & None & None \\
\hline & $\begin{array}{l}\text { Reabsorption of bile } \\
\text { salts }\end{array}$ & None & None & None \\
\hline
\end{tabular}


Table 2: Capabilities of biorelevant dissolution test devices aimed to mimic aspects derived from GI motility

\begin{tabular}{|c|c|c|c|c|c|}
\hline $\begin{array}{l}\text { Test } \\
\text { Device }\end{array}$ & $\begin{array}{l}\text { Control on dosage } \\
\text { form movement } \\
\text { (C) or } \\
\text { physiologically } \\
\text { relevant transfer } \\
\text { (P) }\end{array}$ & $\begin{array}{l}\text { Exposure of } \\
\text { dosage form } \\
\text { to biorelevant } \\
\text { stresses }\end{array}$ & $\begin{array}{l}\text { Constant flow } \\
\text { conditions }(C) \text { or } \\
\text { Physiologically } \\
\text { relevant flow } \\
\text { conditions }(p)\end{array}$ & $\begin{array}{l}\text { Dynamic } \\
\text { changes of GI } \\
\text { environment }\end{array}$ & $\begin{array}{l}\text { Simulation of } \\
\text { interrupted } \\
\text { media } \\
\text { contact }\end{array}$ \\
\hline $\begin{array}{l}\text { Paddle- } \\
\text { bead } \\
\text { method }\end{array}$ & - & - & - & - & - \\
\hline $\begin{array}{l}\text { Rotating } \\
\text { beaker }\end{array}$ & - & - & $+(\mathrm{C})$ & - & - \\
\hline $\begin{array}{l}\text { Stress } \\
\text { test } \\
\text { device }\end{array}$ & $+(C)$ & + & $+(P)$ & - & + \\
\hline ASD & - & - & - & + & - \\
\hline DGM & - & + & $+(\mathrm{P})$ & + & - \\
\hline TIM-1 & $+(\mathrm{P})$ & + & $+(\mathrm{P})$ & + & - \\
\hline
\end{tabular}

[+: parameter can be simulated; - : parameter cannot be simulated] 

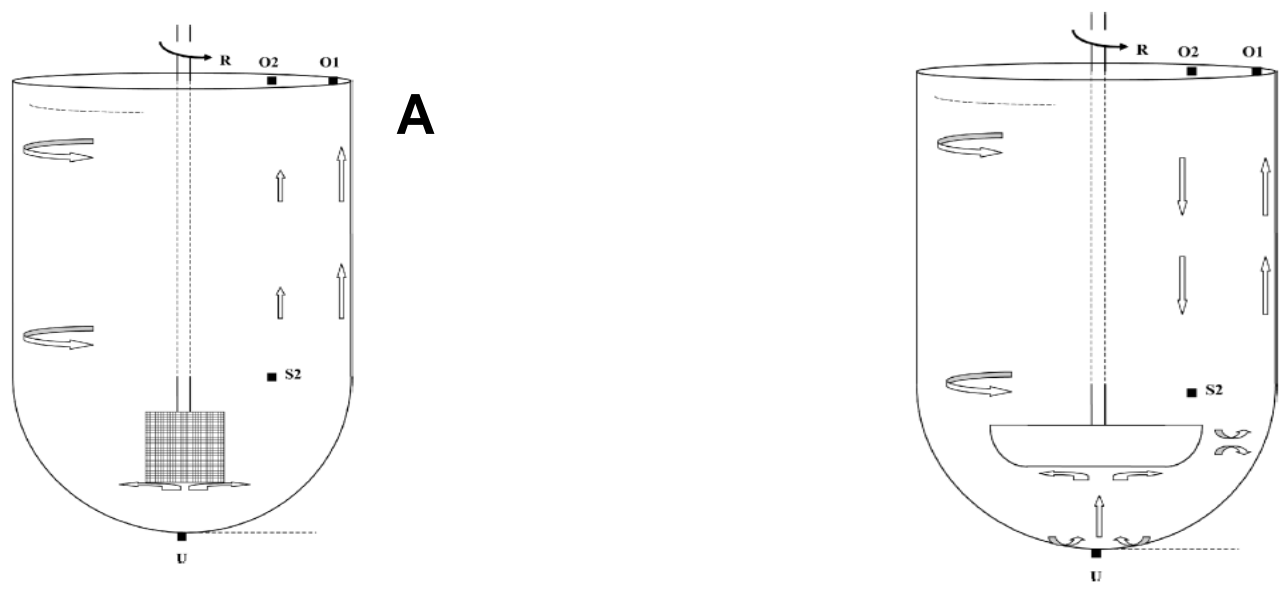

B

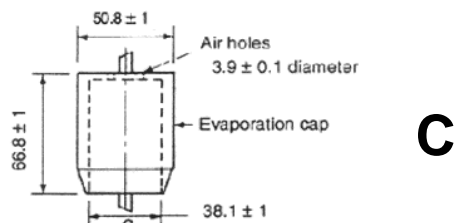

$\rightarrow$ ? $38.1 \pm 1$

Type 316 stainless steel

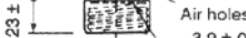

23-26 $3.9 \pm 0.1$ diarnete.

$\longrightarrow 7$ Mes

C
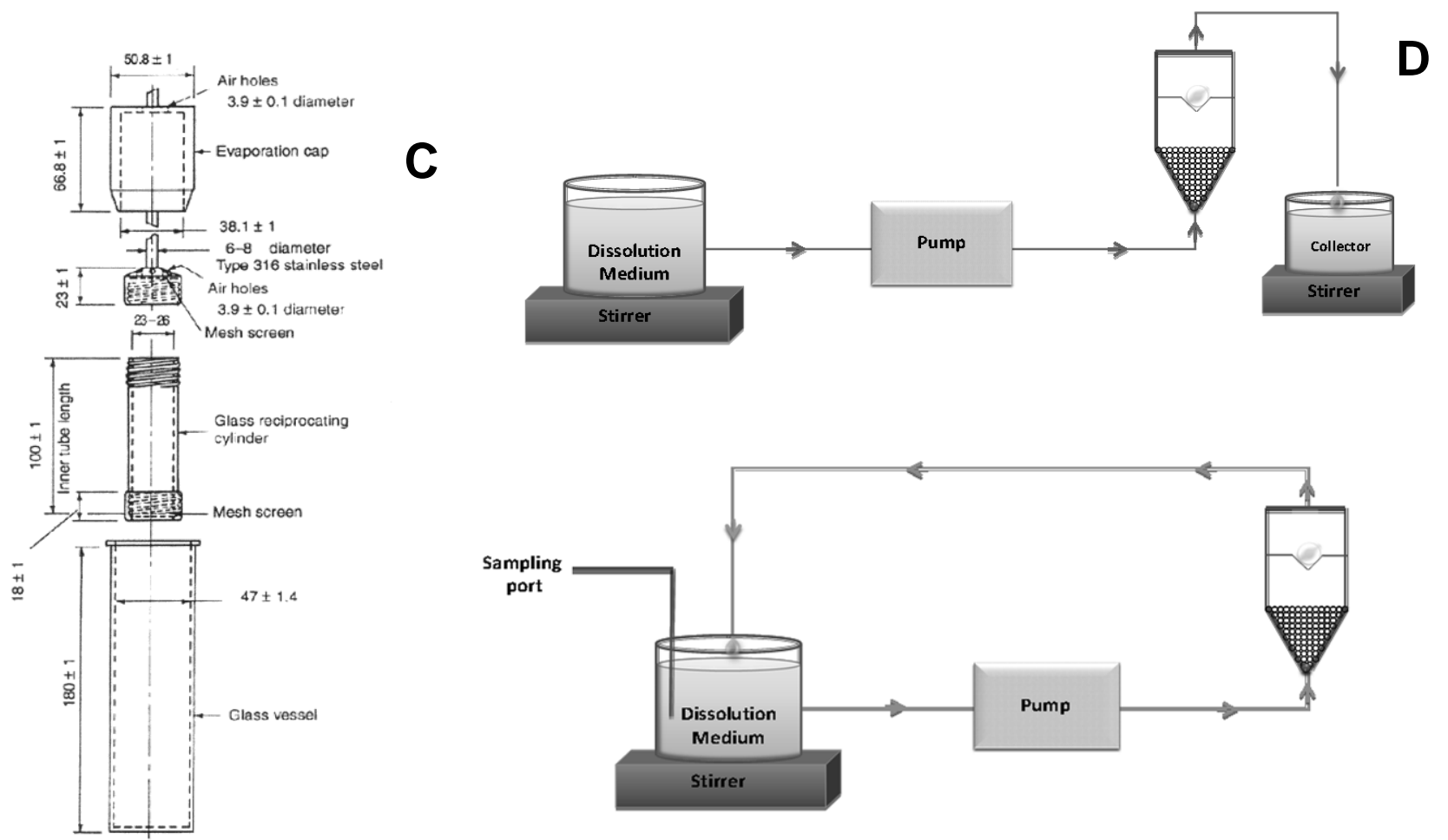
Figure 2
Click here to download high resolution image
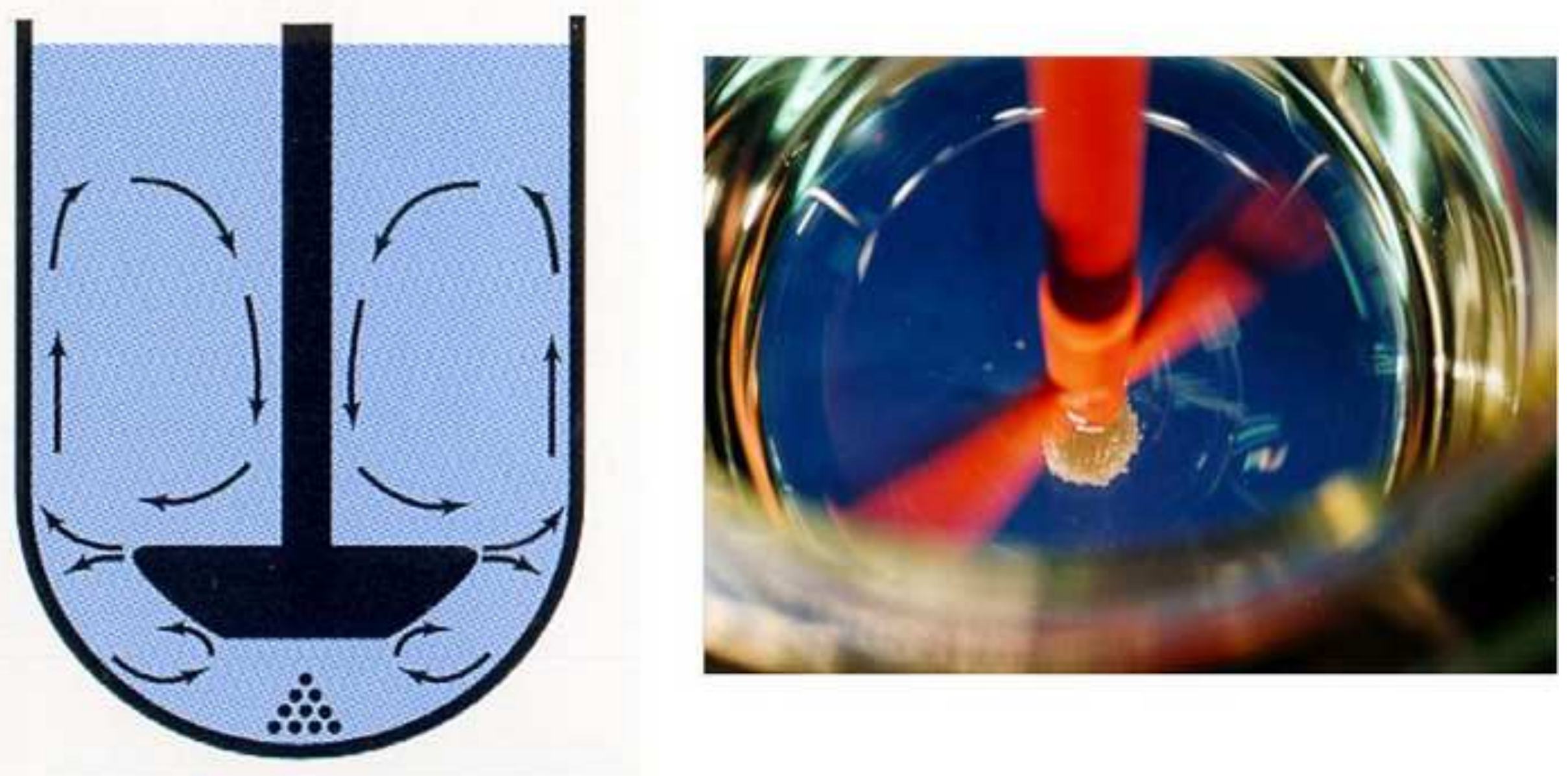


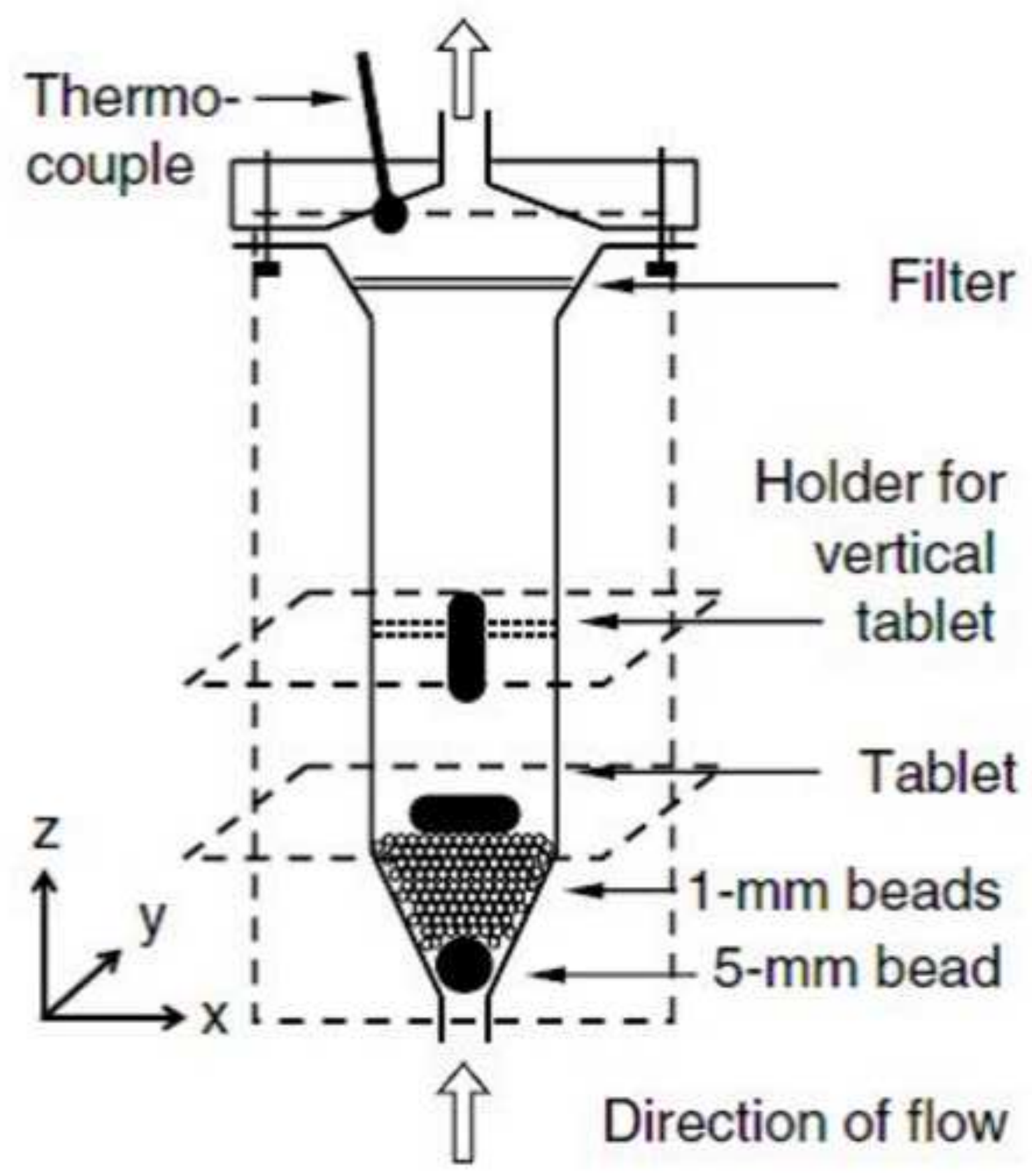


Click here to download high resolution image

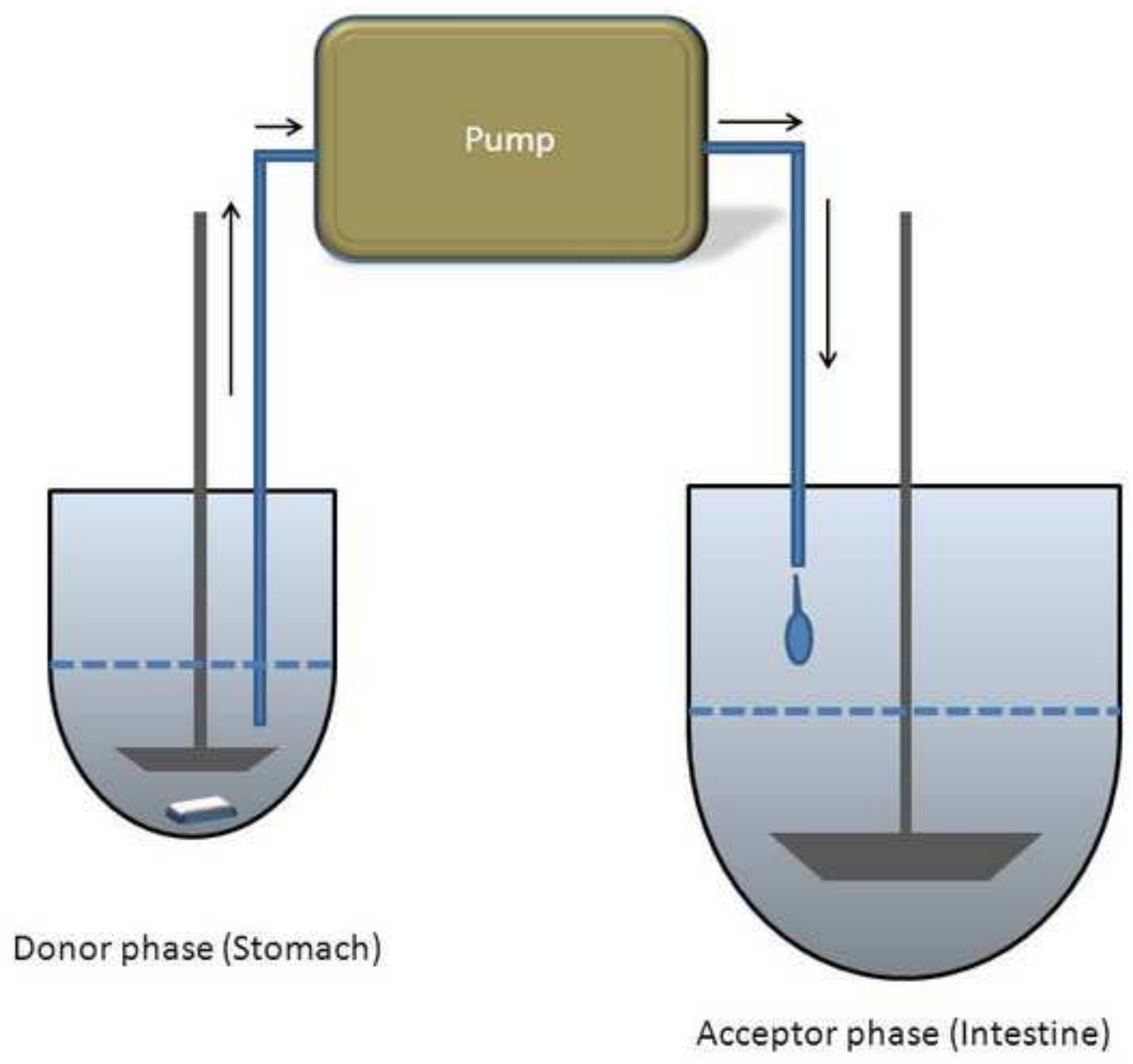


Click here to download high resolution image

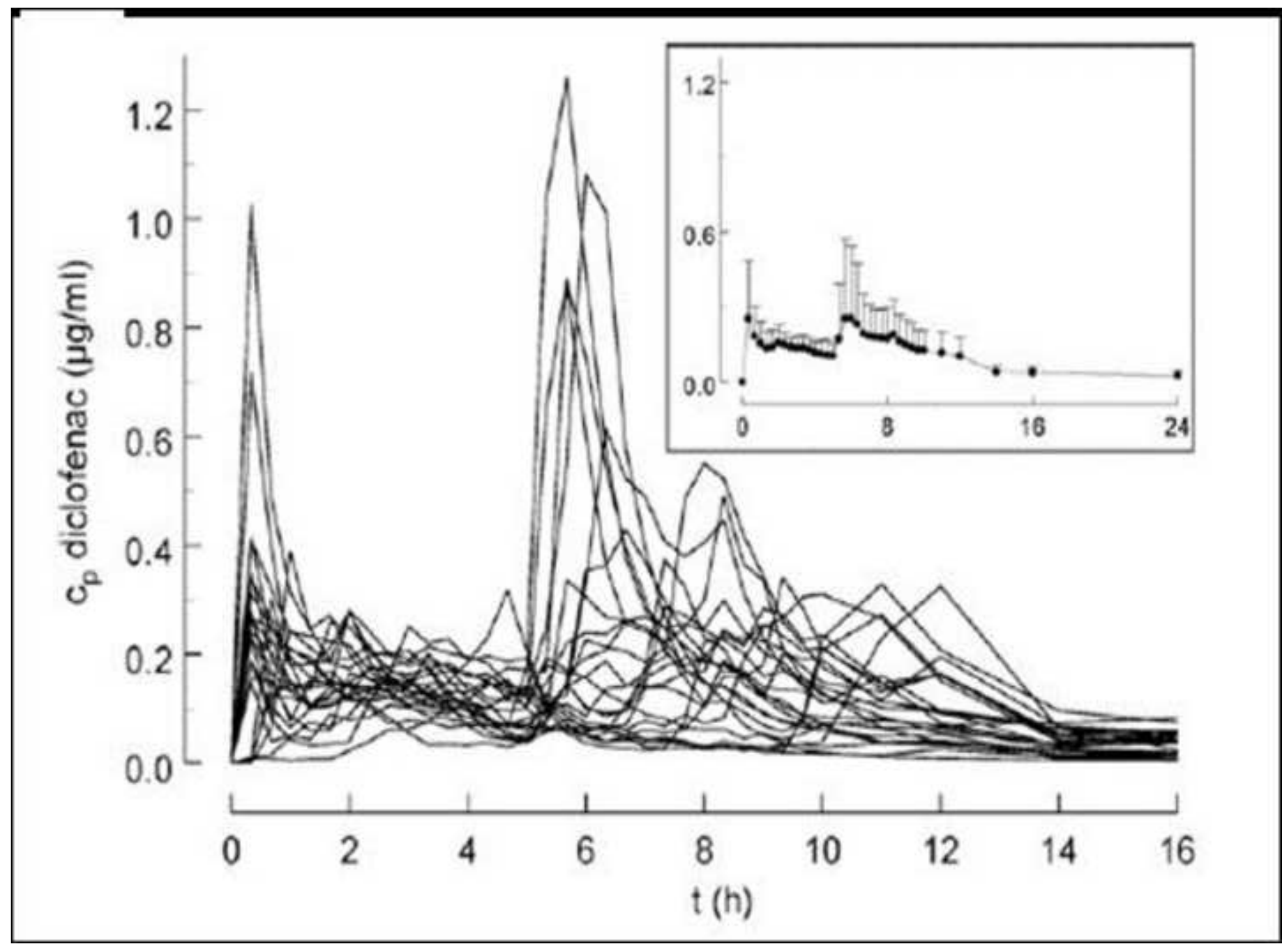




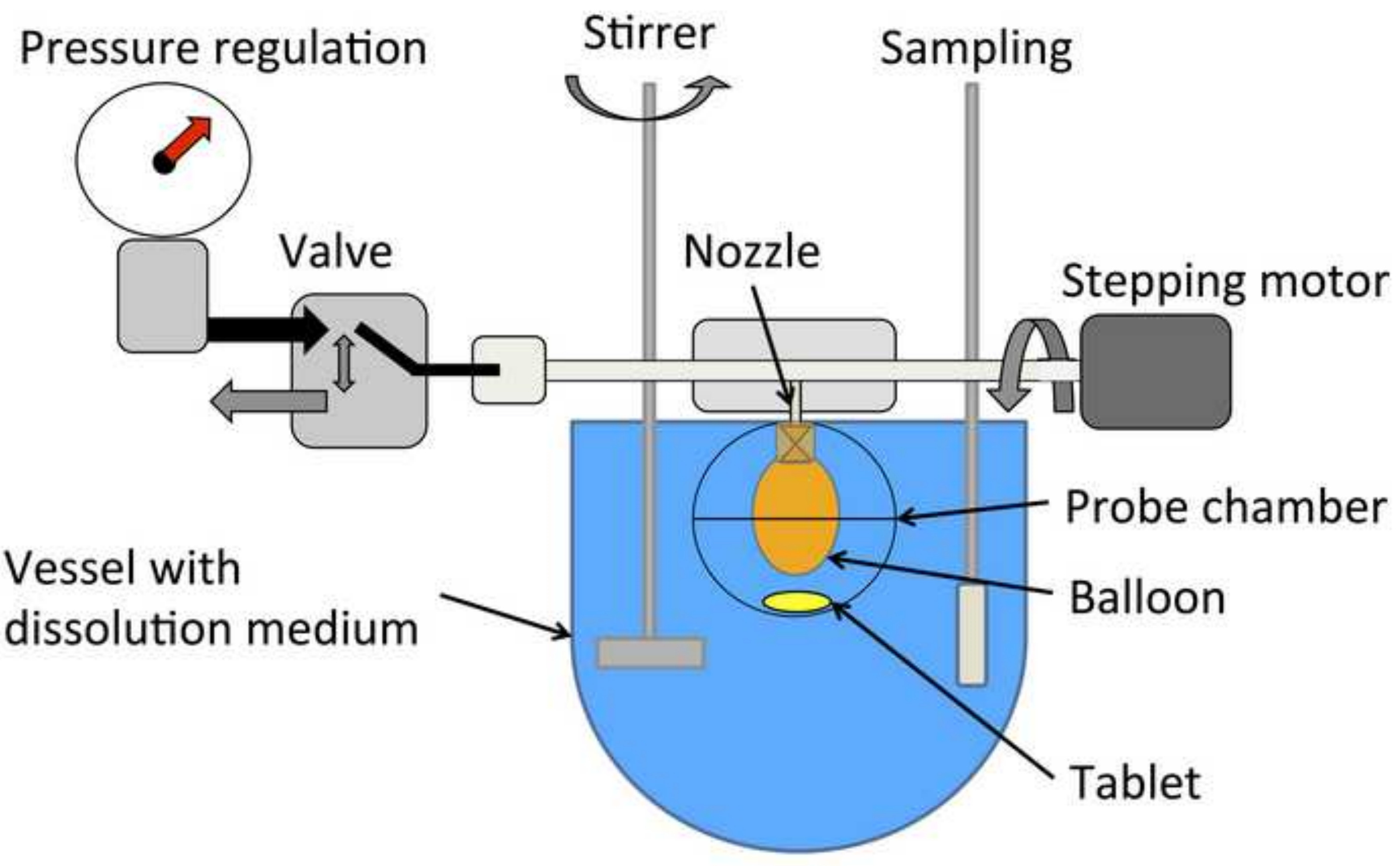




\section{gure 7}

Click here to download high resolution image

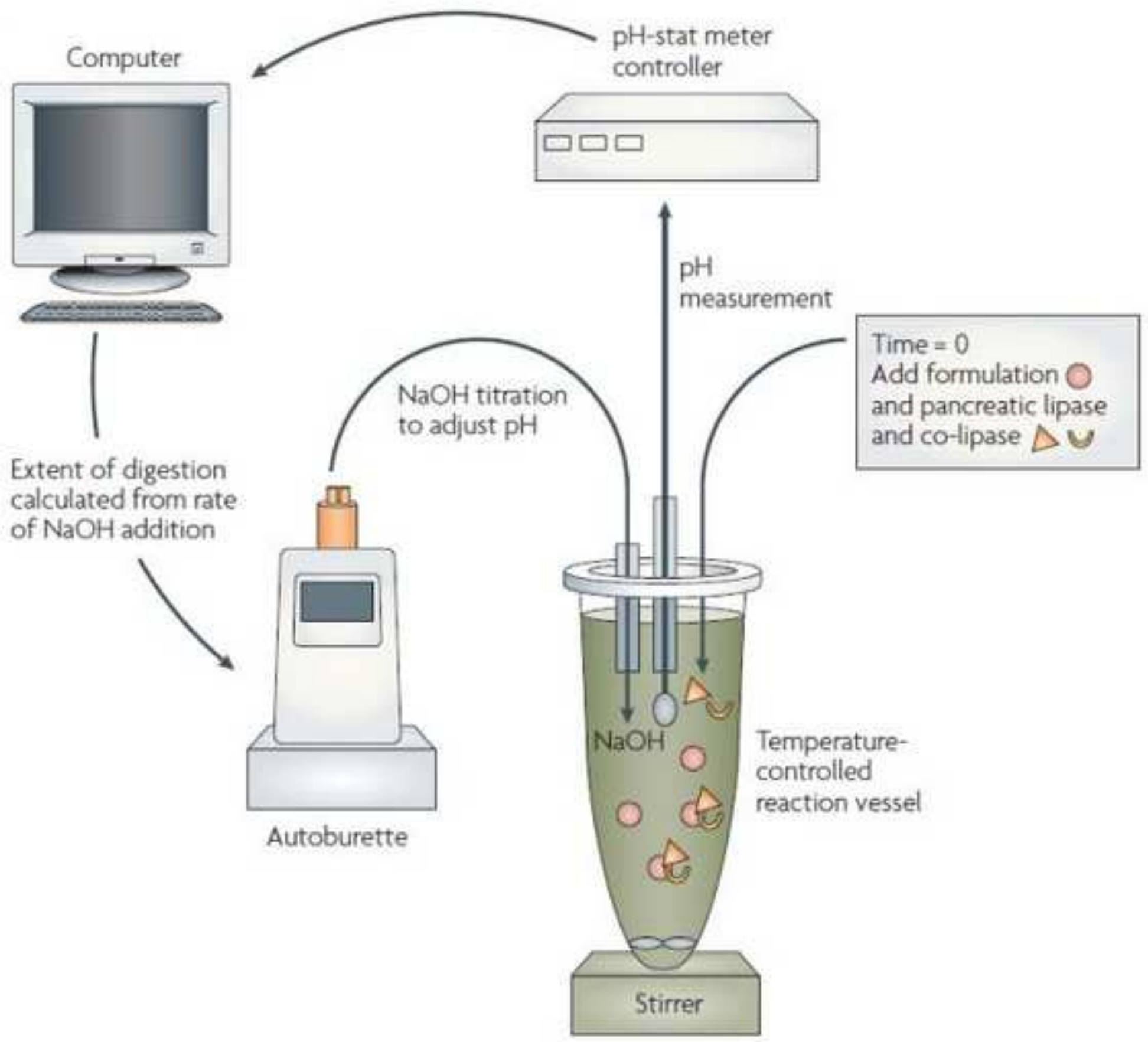


Click here to download high resolution image

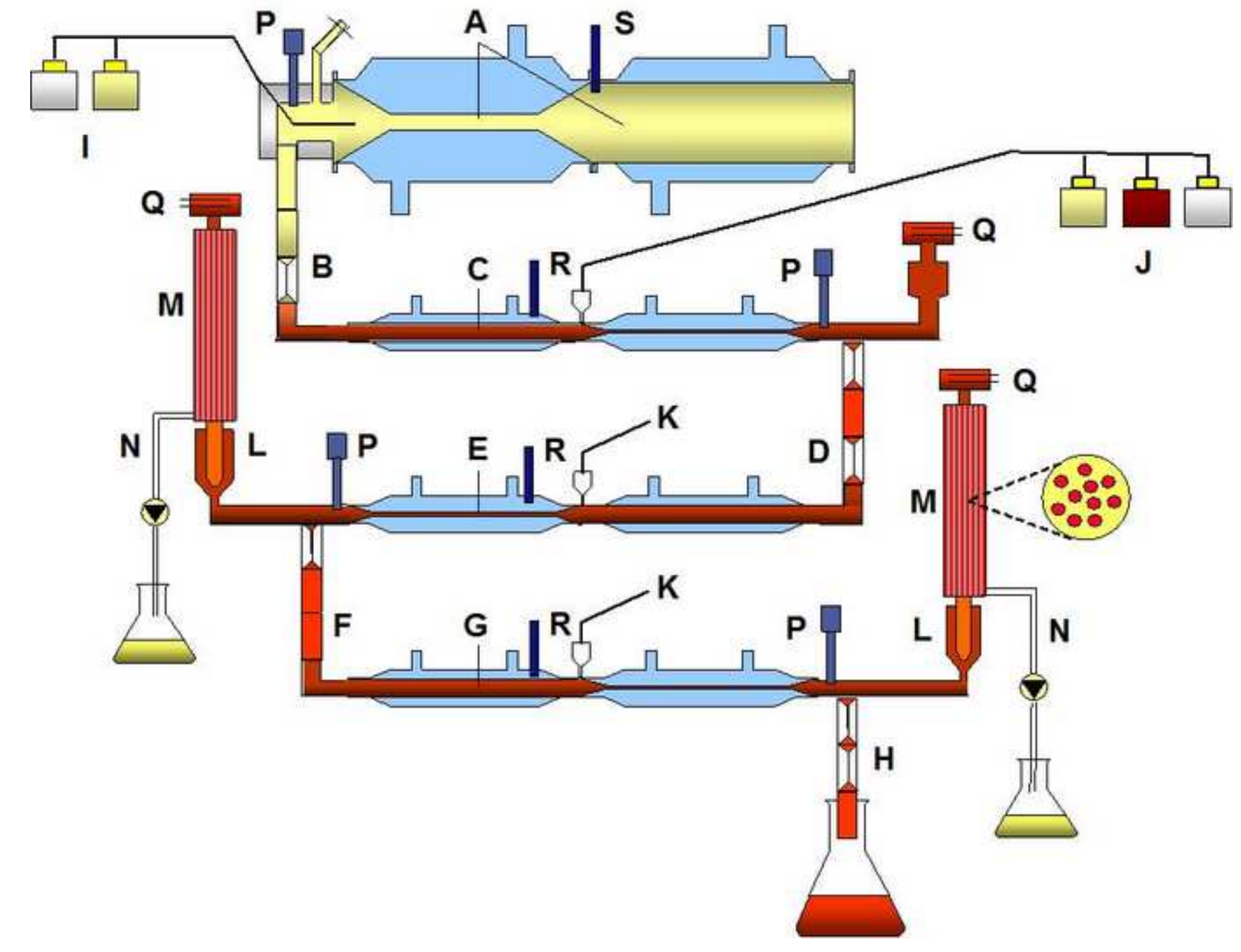

